\title{
Information aggregation in dynamic markets under ambiguity
}

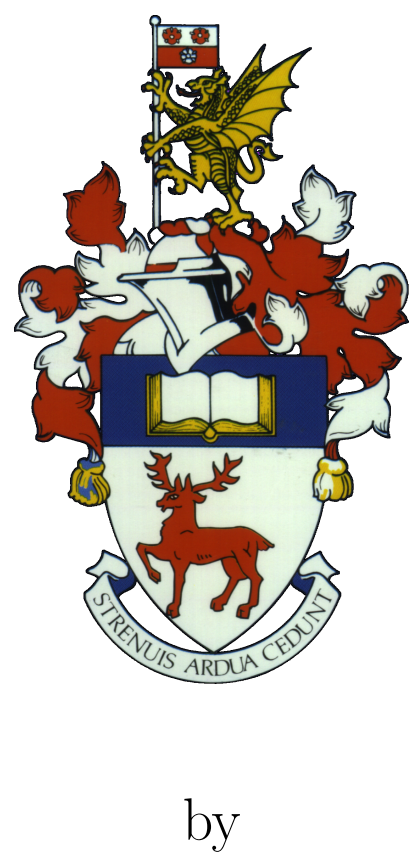

Stelios Kotronis

A thesis submitted in partial fulfillment for the degree of Doctor of Philosophy

in the

Faculty of Social, Human, and Mathematical Sciences

Department of Economics

December 2016 

This Doctoral Thesis is dedicated to my parents for their support. 



\title{
UNIVERSITY OF SOUTHAMPTON
}

\author{
$\underline{\text { ABSTRACT }}$ \\ FACULTY OF SOCIAL, HUMAN, AND MATHEMATICAL SCIENCES \\ DEPARTMENT OF ECONOMICS
}

\section{Doctor of Philosophy}

by Stelios Kotronis

Does ambiguity affect the efficiency of information aggregation in dynamic markets? To the present day there is a sparse and fragmented literature pointing towards an answer. This Thesis studies dynamic markets under ambiguity and examines under what conditions information gets aggregated. Three particular perspectives are investigated:

i) Does information gets aggregated when traders are myopic and ambiguity averse? In the first Chapter it is proved that information gets aggregated only when a "separable under ambiguity" security is traded. In case the security is not "separable under ambiguity", then there exist markets in which information does not get aggregated. The class of "separable under ambiguity" securities is proved to be non trivial. Finally, it is proved that even if the security is not "separable under ambiguity", traders will reach an agreement about the price of the security.

ii) Does information gets aggregated when traders are strategic and ambiguity averse? By defining appropriately an equilibrium concept for infinite horizon games of incomplete information in a setting with ambiguity, it is proved that in a market with a "separable under ambiguity" security information gets aggregated in every equilibrium in pure strategies. The second chapter concludes by proving that when the security is not "separable under ambiguity", then there exists an equilibrium in which information does not get aggregated.

iii) Are the previous theoretical predictions met in real life? In the third chapter a laboratory experiment is included. The experimental design follows the theoretical models of the first two chapters. The results of the experiment provide significant evidence in favor of the results of the first two chapters. 



\section{Contents}

1 Introduction 1

1.1 The theoretical literature of information aggregation . . . . . . . . . 1

1.1.1 "Agreeing to disagree" under subjective expected utility . . . . . . 1

1.1.2 From "agreeing to disgree" to information aggregation . . . . . . . 3

1.1.3 "Agreeing to disagree" under ambiguity . . . . . . . . . . . . 5

1.1.4 Concepts of equilibrium under ambiguity . . . . . . . . . . 6

1.1.5 Discussion . . . . . . . . . . . . . . . . 7

1.1.5.1 From "agreeing to disagree" under ambiguity to information aggregation? ............ 7

1.1.5.2 Equilibrium concept for infinite horizon incomplete information information games ............ 8

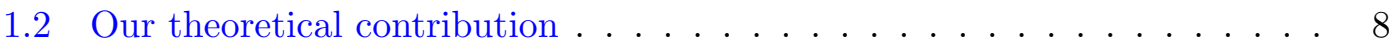

1.2.1 Motivation .................. 8

1.2 .2 Market Microstructure . . . . . . . . . . . . . . . . . . 12

1.2 .3 Results ........................... 13

1.2.3.1 Results for myopic traders . . . . . . . . . . . . 13

1.2.3.2 Results for strategic traders . . . . . . . . . . . . . . . 14

1.3 The experimental literature of information aggregation . . . . . . . . . . 15

1.4 Our experimental contribution . . . . . . . . . . . . . 16

1.4 .1 Motivation ...................... 16

1.4 .2 Results . . . . . . . . . . . . . . . . . 18

2 Information Aggregation with Myopic Traders $\quad 19$

2.1 Introduction . . . . . . . . . . . . . . . . . . . . . . . . 19

2.1.1 Literature Review . . . . . . . . . . . . . . . . . . . 22

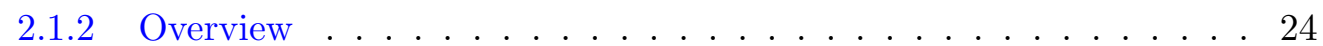

2.1 .3 Trading Environment . . . . . . . . . . . . . . . . . . . . . . . . . . . . . . 24

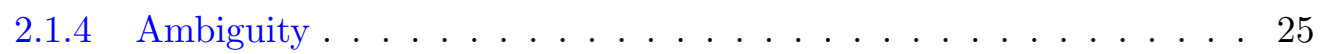

2.1 .5 Myopic Traders . . . . . . . . . . . . . . . . . 26

2.2 Information Aggregation . . . . . . . . . . . . . . . . . . 28

2.3 Separability Under Ambiguity . . . . . . . . . . . . . . . . 28

2.4 Main Result . . . . . . . . . . . . . . . . . . . . . . 31

2.5 Concluding remarks $\ldots \ldots \ldots \ldots$

3 Information Aggregation with Strategic Traders 35

3.1 Introduction . . . . . . . . . . . . . . . . . 35

3.1 .1 Literature Review . . . . . . . . . . . . . . 37 
3.1 .2 Overview . . . . . . . . . . . . . . . . . 38

3.2 Model . . . . . . . . . . . . . . . . . . . . . . . 38

3.2 .1 Equilibrium Concept . . . . . . . . . . . . . . . . . . . 39

3.2.1.1 Equilibrium . . . . . . . . . . . . . . 39

3.3 Information Aggregation . . . . . . . . . . . . . . . . . . . . . . . . . . . . . . . 44

3.4 Separability Under Ambiguity . . . . . . . . . . . . . . . . . . . . . . . 44

3.5 Main Result . . . . . . . . . . . . . . . . . . . . . . . 45

3.5.1 Myopic Behavior can be Revision-Proof . . . . . . . . . . . . . . . 46

3.6 Concluding remarks . . . . . . . . . . . . . . . . . 46

4 Experiment $\quad 49$

4.1 Introduction . . . . . . . . . . . . . . . . . . . . . . 49

4.2 Literature Review . . . . . . . . . . . . . . . . . . . . . . 50

4.3 Experimental Design . . . . . . . . . . . . . . . . . . 52

4.3.1 Risk Vs Ambiguous Box with Security X . . . . . . . . . . 53

4.3.2 Risk Vs Ambiguous Box with Security Y . . . . . . . . . . . 54

4.4 Analysis Metrics and Hypotheses . . . . . . . . . . . . . . . . . 55

4.5 Experimental Results . . . . . . . . . . . . . . . . . . . . . 56

4.5.1 Results without Learning Effects . . . . . . . . . . . . . . . 56

4.5.1.1 Ambiguity Vs Risk for Security X . . . . . . . . . . 57

4.5.1.2 Ambiguity Vs Risk for Security Y . . . . . . . . . 58

4.5.2 Results with Learning Effects . . . . . . . . . . . . . . . . . 58

4.5.2.1 Ambiguity Vs Risk for Security X . . . . . . . . . . 59

4.5.2.2 Ambiguity Vs Risk for Security Y . . . . . . . . . . 60

4.6 Conclusion . . . . . . . . . . . . . . . . . . . 61

5 Conclussion $\quad 63$

$\begin{array}{ll}\text { A } & 65\end{array}$

$\begin{array}{lc}\text { B } & 73\end{array}$

$\begin{array}{lr}\text { C } & 79\end{array}$

$\begin{array}{ll}\text { D } & 93\end{array}$

$\begin{array}{ll}\text { E } & 97\end{array}$

$\begin{array}{ll}\text { Bibliography } & 99\end{array}$ 


\section{List of Figures}

1.1 Private signals. . . . . . . . . . . . . . . . . . . 9

4.1 Treatments. . . . . . . . . . . . . . . . . 52

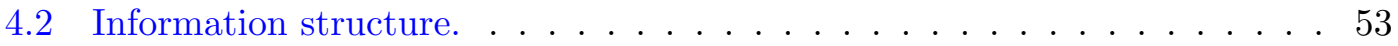

4.3 Discounting. . . . . . . . . . . . . . . . . 54

4.4 Box plots for treatments with security X. . . . . . . . . . . 56

4.5 Box plots for treatments with security Y . . . . . . . . . 57

4.6 Permutation tests for treatments with security X . . . . . . . . . 57

4.7 Permutation tests for treatments with security Y . . . . . . . . . 58

4.8 Learning patterns for treatments with security X. . . . . . . . . . . . . 59

4.9 Learning patterns for treatments with security X. . . . . . . . . . . . . . 59

4.10 Box plots incorporating learning effects. . . . . . . . . . . . . 60

4.11 Permutation tests for treatments with security X . . . . . . . . . 60

4.12 Permutation tests for treatments with security X . . . . . . . . . 60

E.1 Experimental Data for Security X. . . . . . . . . . . . . . . 97

E.2 Experimental Data for Security Y. . . . . . . . . . . . . . . . 97 



\section{Academic Thesis: Declaration of Authorship}

I, Stylianos Kotronis, declare that this thesis and the work presented in it are my own and has been generated by me as the result of my own original research.

Title of thesis: Information aggregation in dynamic markets under ambiguity.

I confirm that:

1. This work was done wholly or mainly while in candidature for a research degree at this University;

2. Where any part of this thesis has been submitted for a degree or any other qualification at this University or any other institution, this has been clearly stated;

3. Where I have consulted the published works of others, this has always been clearly attributed;

4. Where I have quoted from the work of others, the source is always given. With the exception of such quotations, this thesis is entirely my own work;

5. I have acknowledged all main sources of help;

6. Where the thesis is based on work done by myself or jointly with others, I have made clear exactly what was done by others and what I have contributed myself;

7. none of this work has been published before submission 



\section{Acknowledgements}

First and foremost, I would like to express my gratitude to my supervisor, Spyros Galanis, for his guidance, his help and his support. I am grateful to him for his time, his ideas and his suggestions. In addition, I would like to thank him for introducing me in such an interesting area of Economics.

I would also like to thank my co-supervisors, Michael Vlassopoulos and Miltiadis Makris for their help, their time and their interesting comments during our discussions.

I want to thank all the postgraduates and postdocs I have come across since I have been in Southampton. All of them made me feel welcome since my first day. Thank you all, guys.

I would also like to thank my friends, Dionisis, Dimitra, Konstantinos, Liu for the nice times and discussions. Thank you Michael and George for the discussions and your help in my third Chapter.

Maria has stood by me with devotion and love and has helped me in more ways than she can imagine. I cannot thank her enough for or everything that she has offered to me.

Thank you to the Department of Economics at the University of Southampton and the Economic and Social Research Council for awarding me with a scholarship to pursue this Thesis.

Finally, I owe my deepest gratitude to my family; my father Panagiotis my mother Paraskevi, and my sister Georgia. They have always encouraged and supported my dreams and aspirations and have surrounded me with love and affection. Without them, this work could not have been completed. 



\section{Chapter 1}

\section{Introduction}

This chapter presents an overview of the literature which is related to information aggregation. An extensive survey of theoretical and experimental results and contributions are exhibited, which are closely related to our results. The scope of this chapter is to indicate the aim of this doctoral thesis and to distinguish its position in the theoretical microeconomic and experimental literature.

In more detail, Section 1.1 documents the evolution of some building blocks of the information aggregation literature in such a way, such that, the contribution of the thesis to be understandable. Section 1.2 proceeds by motivating the first two chapters of the thesis and by presenting the main results. In Section 1.3 we document parts of the experimental literature related to information aggregation. In Section 1.4 we present the motivation for our third chapter, and its results.

\subsection{The theoretical literature of information aggregation}

In this section of the thesis we will report the literature of information aggregation. We will extensively analyse and report the part which is more related to the focus of the two first chapters of our thesis, so that our contribution to be comprehensible and positioned easily within the literature.

\subsection{1 "Agreeing to disagree" under subjective expected utility}

The concept of common knowledge was firstly formalised in the seminal result of Aumann (1976). The result states that when the opinions of two traders are common knowledge, which intuitively is the same as assuming that the opinions are publicly known to all traders, then traders cannot disagree, assuming a common prior. The main argument underlying this result is the additivity property of probability measures. 
However, the result of Aumann (1976) was static. It does not explain how the common knowledge of opinions is met. Geanakoplos \& Polemarchakis (1982) are arguing that when two traders are exchanging their opinions sequentially then, under the assumption of a common prior and the common knowledge of their information partitions, they will finally reach the common knowledge event. Hence, using the additivity property of probability measures they prove that in the common knowledge event they have to agree. While in Geanakoplos \& Polemarchakis (1982) several examples are presented, there is not formally studied the question of information aggregation but rather the question of consensus.

Following these two results, a considerable amount of literature studies these type of results under various modifications of the initial assumptions and setting.

Cave (1983) tries to generalise the result of Geanakoplos \& Polemarchakis (1982) by leaving the assumption of common prior, but incorporating the property of union consistency. Intuitively, this is a property that mimics the additivity of probability measure, hence allowing for similar arguments in order to conclude the agreement. Bacharach (1985) used the same property, naming it as Sure Thing Principle, as in Geanakoplos (1995), too.

Nielsen (1984) argues that the setting of Aumann (1976) and Geanakoplos \& Polemarchakis (1982) is restrictive, as they both assume finite states space. In Nielsen (1984) a result is given using sigma algebras.

However, all these contributions are referring to a setting in which traders are exchanging opinions in the form of probability assessments of an event. In Nielsen et al. (1990) the result of Geanakoplos \& Polemarchakis (1982) is generalised. A security over a state space is assumed and traders are exchanging opinions in the form of the expected value of the security. Although it is more generalised setting the main argument is still the linearity of the expected value. Similarly, Sebenius \& Geanakoplos (1983) argue that in a betting game the private information of the traders will be aggregated in such a way that, under the assumption of common prior, the bet is not going to be mutually beneficial.

Conceptually similar, to Sebenius \& Geanakoplos (1983), results are given in Milgrom \& Stokey (1982). In this paper the seminal no trade theorem is proved, which proves the impossibility of speculative trade. In particular, it states that when two traders agree on an ex ante allocation, then in the presence of new information there is not common knowledge trade. Intuitively, the result is technically based on the additivity of the common prior probability measure, too.

The literature we presented so far, contributes in the information aggregation literature by establishing results which try to explain how the private information of traders are aggregated in a statistic, which is (becoming) common knowledge. However, until that 
point none of these papers studied under what conditions the aggregate statistic reveal information about the fundamental of a stock.

Such a result has been given in DeMarzo \& Skiadas (1998) and DeMarzo \& Skiadas (1999). In particular, they studied competitive equilibria within a setting similar to that of Nielsen et al. (1990). They proved that, as opposed to Radner (1979) (fully revealing $\mathrm{REE}$ ), these competitive equilibria lead to the generic existence of Common Beliefs Equilibria in which prices, in equilibrium, do not fully aggregate traders information. They proved that in order to fully aggregate traders' information the security should be a separably oriented security. Intuitively, the idea underlying this property is that when traders reach the common knowledge event then at least one should be non-trivially informed, unless the common knowledge event is such that as if traders pooled their private signals.

\subsubsection{From "agreeing to disgree" to information aggregation}

The literature presented in Section 1.1.1 is consisted of pure theoretical results. The opinion games of Geanakoplos \& Polemarchakis (1982) and Aumann (1976) is not met, per se, in practice, hence leaving a gap between strong theoretical results and the lack of their practical implications.

Hanson (2003) defined a market structure, which is more and more used in practical applications. The market is called Market Scoring Rule. Its basic building block is a strictly proper scoring rule. In fact, this is a function which gives incentives to agents to reveal their true beliefs regarding an event. This is a property known as myopic incentive compatibility.

A strictly proper soring rule, when adopted to a setting similar to Nielsen (1984) is myopic incentive compatible, too. This result was proved, among others, in Chen et al. (2012) and Ostrovsky (2012). This fact implies that when a trader, being privately informed, wants to maximise his/her expected utility, then a strictly proper scoring provides those incentives so that the maximum is achieved by the trader announcing the expectation of the security conditional to their private information.

Hence Hanson (2003) provides a market model which, essentially generalises, hence implying, the communication process of Geanakoplos \& Polemarchakis (1982). Formally, this is true in case we assume that traders participating in the market are myopic.

In particular, considering a setting as in Nielsen (1984), the accordingly modified Market Scoring Rule of Hanson (2003) considers two traders participating sequentially and repeatedly in a market. Each trader announces his/her prediction regarding the value of the security. Therefore, given that their payments are depending on a strictly proper scoring rule and assuming that both are trying to maximise their per period utility, 
hence they are not strategic but myopic, it is implied that at each period they announce the expected value of the security condition to their private information. This market is compelling because this sequential announcement of prediction can be interpreted as buying or selling the security to an automated market maker.

This model is formally built in Chen et al. (2012). In particular, the paper modifies the Market Scoring Rule of Hanson (2003) according to a setting similar to Nielsen et al. (1990). It considers a security and an information structure, over a finite state space. Its aim is to prove that under some conditions this market not only reaches a consensus, as it was proved in Nielsen et al. (1990), but also aggregates information. This is defined as traders know finally the true value of the security. The information aggregation result is achieved by restricting to a class of securities, with similar properties as in DeMarzo \& Skiadas (1998) and DeMarzo \& Skiadas (1999). This class was defined in Ostrovsky (2012) and the securities belonging to it are called separable. In this thesis, from this point and onwards, we will use, instead, the term separable under SEU (subjective expected utility). The intuition of the argument, which concludes information aggregation, in Chen et al. (2012), is that when the agreement is reached, hence no trader holds private information not revealed in the market, then the properties of separable under SEU securities imply that the common knowledge event is consisted of exactly those states which value the security the same as the realised state does. In addition, Chen et al. (2012) proves that this result holds even if multiple securities are traded in the market. A similar result of information aggregation was given in McKelvey \& Page (1986a), under the assumption that securities are belonging to a subclass of separable under SEU securities.

In Ostrovsky (2012) two market models are used. The first one is Kyle (1985) and the other is Hanson (2003). The model of Hanson (2003) is similar to the respective model of Chen et al. (2012). The main contribution of Ostrovsky (2012) is that it is proven that the myopic result of information aggregation under separable under SEU securities still holds when traders are strategic. In particular, it is proved that in every Nash equilibrium in a Market Scoring Rule with a separable under SEU security information gets aggregated.

Grossman (1976) was, among the firsts, to establish results related to information aggregation in markets, without providing results for case when traders are strategically large compared to the market. The same applies for Radner (1979), where the Rational Expectations Equilibrium is introduced. Several results for models of decentralised trading studied the convergence to REE, Golosov et al. (2014), Dubey et al. (1987), Wolinsky (1990) to name a few. However, in these papers, each trader's behavior effect on how the trading is processed is not taken into account. Hence restricting the strategic behavior. On the other hand, Ostrovsky (2012) considers a finite number of traders, who are large and affect the evolution of trading process. 
Furthermore, strategic interactions and their effects on information aggregation was studied in models as formalised in Hanson (2003), hence simplifying the setting of Nielsen (1984). In particular, Chen et al. (2010) undiscounted finite games are considered. The games are based on a logarithmic strictly proper scoring rule. Their result states that when traders hold private signals which are independent conditional on the value of the security, then it is an equilibrium for each trader to predict their conditional beliefs, or in other words myopically.

On the other hand, Dimitrov \& Sami (2008) similar games were considered. In contrast to Chen et al. (2010) the games here are infinite. In addition, traders' signals are independent. Their results suggest that, in the Market Scoring Rule the game with no discounting, predicting their conditional beliefs is not, in principle, an equilibrium. They provided a simple Market Scoring Rule game with discounting, and showed that information gets aggregated, when we assume that the complementarity bound of the security is positive.

\subsection{3 "Agreeing to disagree" under ambiguity}

The result of Aumann (1976) has triggered a line of research trying to generalise it in many ways. Among other dimensions, in this subsection we will report results that refer to a setting with ambiguity.

Kajii \& Ui (2005) and Kajii \& Ui (2009) are establishing a version of Aumann (1976), under the assumption of Gilboa \& Schmeidler (1989). Although the scope of these papers is not exclusively to study agreement in a setting with ambiguity, they both provide conceptually the same agreement result. They argue that the agreement under ambiguity means each traders' "opinion" sets, they have a set of opinions because they assume the multiple priors model of Gilboa \& Schmeidler (1989), have no empty intersection.

Similar perspective is followed in Carvajal \& Correia-da Silva (2010). In fact, this paper tries to reformulate the results Kajii \& Ui (2005) and Kajii \& Ui (2009). In Carvajal $\&$ Correia-da Silva (2010) it is argued that the assumption of common set of priors, existing in Kajii \& Ui (2005), can be dropped. It is proved that in order traders not agree to disagree the non emptiness of the traders set of priors is sufficient. However, this observation was made in Kajii \& Ui (2009), but by restricting the sets to be convex and compact. However, in Carvajal \& Correia-da Silva (2010) it is proved that only the connectedness of the sets of priors is enough to prevent disagreement. Finally, Carvajal \& Correia-da Silva (2010) provides the results considering both prior by prior Bayesian and the maximum likelihood update rule, Pires (2002).

On the other hand, results, for a setting with ambiguity, were given under the concept of agreement as defined in the classical literature presented in Section 1.1.1. In particular, in Dominiak \& Lefort (2015) the agreement theorem is proved for capacities, as defined 
in Schmeidler (1989). In fact, it tries to generalise the result of both Aumann (1976) and Nielsen (1984), by establishing results both for the agreement in beliefs and in conditional expectation given a security. The main idea underlying Dominiak \& Lefort (2015) is conceptually similar to the property of union consistency, Cave (1983) and Bacharach (1985), which in turn is the same with the Sure Thing Principle of Geanakoplos (1995). In more detail, as we have mentioned in Section 1.1.1, the additivity of the common prior probability measure was the main argument to conclude the agreement. However, under ambiguity this property does not hold, as in Cave (1983) and Bacharach (1985), hence, assuming union consistency in order to "mimic" the additivity property. By doing so agreement is implied. Finally, in Dominiak \& Lefort (2015), the union consistency is related to the definition of unambiguous events, Dominiak \& Lefort (2011).

In Dominiak \& Lefort (2013) the agreement theorem is studied by focusing on neoadditive capacities, Chateauneuf et al. (2007). Firstly, it is proved that agreement is not possible when their posterior capacities are common knowledge in some event. Secondly, it characterises the updating rule so that the agreement theorem to hold.

\subsubsection{Concepts of equilibrium under ambiguity}

Two lines of research can be identified within the literature of games under ambiguity. Firstly, a big class of games that are studied are those in which ambiguity is about the type space, or the state space. The second class of games are those in which the states are unambiguously determined, but there exists ambiguity regarding the strategies of the opponents. The game defined in this thesis belong to the first class.

In Bose \& Renou (2011) and Bose \& Daripa (2009) the effect of ambiguity aversion is studied in a mechanism design framework. The games of these papers are very closely related to our approach. However, their games are of finite horizon. The equilibrium concept defined is the consistent planning equilibrium. According to it, traders are optimising over one stage deviations, given that afterwards they follow their equilibrium strategies. In Bose \& Renou (2011) and Bose \& Daripa (2009) the off equilibrium beliefs are left unconstrained, which in fact makes it conceptually more similar to the Weak Perfect Bayesian Equilibrium and not Perfect Bayesian Equilibrium. A similar equilibrium concept is used in Mele \& Sangiorgi (2015).

In Hanany et al. (n.d.) a more abstract approach is taken. The paper suggests two equilibrium concepts, namely Sequential Equilibrium with Ambiguity and Perfect Equilibrium with Ambiguity, and existence results are given. However, the games have finite horizon. The idea underlying these equilibrium concepts is to have a component of sequential rationality (as in the standard subjective expected utility case) and a second component that determines the way that beliefs are updated. Although this is not in general feasible when consequentialist updating rules are used, here the updating rule 
follows the results of Hanany \& Klibanoff (2009), hence dynamic consistency holds. Therefore the sequential rationality is well defined. As a result of the updating rule, it is proved that the rationality component is equivalent to the one-shot-deviation principle, relating it, in that sense, to the consistent planning equilibrium.

Furthermore, dynamic games are studied as well in Eichberger \& Kelsey (2004) and Dominiak \& Lee (n.d.), providing applications to signaling games. The models in these papers allow for both environmental and strategic ambiguity. The novelty of their paper is that the Dempster - Shafer updating rule, which they suggest, can be used to update the beliefs even after deviations.

In Azrieli \& Teper (2011), a result relating the existence of equilibrium and the direction of aversion, ambiguity aversion or ambiguity loving, is established. In particular, it is proven that for an equilibrium to exist, players should be ambiguity averse. However, no results are given for a dynamic game. In fact, the union consistency is proposed as a potential way to overcome the dynamic inconsistency in the dynamic case.

Finally, in Sass 2013 ambiguity is introduced with regards to the opponents' strategies. By defining the Ellsberg strategies, which intuitively are mixed strategies but using an Ellsberg urn for the randomisation, they derive a form of the Kuhn theorem.

\subsubsection{Discussion}

In the two subsections that follow we discuss the gaps of the related literature. The ultimate aim is to signify how Chapter 2 and Chapter 3 contribute and improve the literature, and how they fit within it.

\subsubsection{From "agreeing to disagree" under ambiguity to information aggre- gation?}

Comparing Section 1.1.3 with Section 1.1.1 we can observe that there exists a gap in the literature of information aggregation under ambiguity. Although it followed and generalised, in some extent, the classical results of "agreeing to disagree", there are no results directly related to how and under what conditions information gets aggregated in a market setting like Hanson (2003).

In particular, results are lacking in the direction of generalising Chen et al. (2012) and Ostrovsky (2012) under ambiguity. These results would generalise, as a consequence, the literature related to Geanakoplos \& Polemarchakis (1982) and Nielsen et al. (1990), too. 


\subsubsection{Equilibrium concept for infinite horizon incomplete information in- formation games}

The literature of games under ambiguity does not provide an equilibrium concept for infinite horizon incomplete information games, when prior by prior updating, hence a consequentialist updating rule, is used, which refines, as well, consistent planning concept.

In our case consistent planning is not enough in order to conclude our strategic result in Chapter 3. Hence a stronger equilibrium concept should be defined and used. By doing so we indeed can have results similar to Ostrovsky (2012).

\subsection{Our theoretical contribution}

The aim of section is to signify, in detail, the main focus of Chapter 2 and Chapter 3 of this doctoral thesis. The importance of our contribution will be clarified in Section 1.2.1. In addition the model will be reported, intuitively, indicating its position in the literature.

\subsubsection{Motivation}

When traders are participating in a market, they usually hold some private information about the traded security. The price of the security, as affected by the competition, might reveal to some traders the private information of the others. The fact that price acts as a statistic which aggregate the private information of decentralised traders, has been emphasized and extensively studied in economics, with Hayek (1945) to be the first one.

In other words, if information is dispersed among all traders, will their trading behavior lead the market to aggregate information, pricing the securities as if their information was pooled? The main intuition of information aggregation is arbitrage. Informed traders will correct potential mispricings, hence moving security's price to the correct one. This is an attractive intuition when traders are fully informed. Results have been given regarding the case in which traders are partially informed, or even for finite number of large strategic partially informed traders. But what if, in addition, traders are ambiguity averse? Does ambiguity affect the efficiency of information aggregation?

While financial markets have, in general, several other important properties, there are some particular models of financial markets that are trying to leverage their information aggregation property. These markets are also known as information markets or prediction markets. In fact, in these markets a security, contingent to an event, is traded. 
Through the revelation of information, due to trading, traders' signals are pooled into the price. Hence, by correctly pricing the security, a forecast for the event is implied, too.

Many firms and institutions are using prediction markets as a forecasting tool for several issues, such as political events or the release of new products. Among them, several Internet based predictions markets exist. ${ }^{1}$ Among several ways to implement a prediction market, Market Scoring Rule (MSR) is used, more and more, in practice as corporate governance tools. These markers are fairly general as they can be reinterpreted in order to correspond to the classic approach with an inventory based market maker who continuously adjusts the price of the securities, as traders buy from him and sell to him. ${ }^{2}$

In Ostrovsky (2012) and Chen et al. (2012) results are given regarding the conditions under which information is aggregated, in an MSR environment with expected utility and common priors. In particular, the class of separable under $S E U$ securities is defined. When such a security is traded, among either strategic or non-strategic myopic traders with common prior, then the information revealed in the market is such that as if traders have pooled their private signals. An example of a separable under SEU security is the Arrow-Debreu security, which takes the value 1 for a particular state of the world and 0 otherwise.

The intuition of these results can be understood by the following example. Consider an institution setting up a prediction market in order to forecast whether the referendum will take place, and if so its outcome. ${ }^{3}$ Consider security $X$ which takes the value 1 when Brexit happens, and 0 otherwise, when the UK continues being a member of the EU or when the UK government cancels the referendum. Two experts are participating in that market, each announcing his expected utility of $X$, with private signals as shown in Figure 1.1.

\begin{tabular}{c|c|c}
\cline { 2 - 3 } \multicolumn{2}{c}{} & \multicolumn{2}{c}{ Expert 1 } & Expert 2 \\
\hline Brexit & Referendum Not Canceled & Either Brexit or Canceled \\
\hline No Brexit & Referendum Not Canceled & No Brexit \\
\hline Cancel Referendum & Referendum Canceled & Either Brexit or Canceled \\
\hline
\end{tabular}

Figure 1.1: Private signals.

The signals represent the information that the experts hold depending on the realised state of the world. In particular, when the realised state is Brexit, Expert 1 knows

\footnotetext{
${ }^{1}$ Many firms have organised internal prediction markets using models similar to the one we follow in this paper. Google, Ford and General Electric, HP among others run internal prediction markets as a corporate governance and predictions tool. Cultivate Labs, Inkling Markets, Consensus Point, Crowdcast and Iowa Electronic Markets are, among others, some examples of real life prediction markets.

${ }^{2}$ See in Appendix C for more details.

${ }^{3}$ For simplicity we do not assume a prediction made by the market maker, while we assume it in our model. However, even with that version of MSR our results remain robust. In fact, by considering the logarithmic market scoring rule and myopic traders we can conclude that information does not get aggregated for the case that Brexit is the realised state.
} 
that the referendum will not be canceled. Similarly, Expert 2 knows that either Brexit will happen or the referendum will be canceled. Under that information structure, information aggregation means both knowing, finally, the value of the security.

Suppose that Brexit is the realised state and that experts hold a common prior which assigns non zero probability to the event that Brexit happens. Then, by announcing sequentially each other their expectations about the value of security $X$, each can realise what private signal the other expert holds. In the first round, the announcement of Expert 1 will be non zero, hence Expert 2 knows that the private signal of Expert 1 should be that the referendum will not be canceled. In the second round, Expert 2, by combining the extra information with his own private signal, realises that Brexit will happen and announces 1. In the third round, Expert 1 realises, as well, that Brexit is the realised state, hence the prediction market aggregates information.

As it is apparent from the intuition, the result above depends on the fact that there is a common prior between the experts. However, no historical data exist regarding the event to be predicted, hence making the event under consideration not to be quantifiable probabilistically but ambiguous.

In fact, we can observe that this market does not necessarily aggregate information when traders hold ambiguous beliefs, even if they are common. Indeed, suppose the multiple prior model of Gilboa \& Schmeidler (1989), according to which a decision maker would act as if having multiple priors, over the states, and chooses the prediction that maximizes the minimum expected utility over these priors. Assuming that at least one prior (but not all) assigns zero probability to Brexit, the expected value of the security with respect to that prior is 0 . Therefore, the initial announcement of Expert 1, who is ambiguity averse, is his most pessimistic, and because he holds a prior assigning 0 to Brexit, his announcement is 0 . Hence the next trader cannot infer whether the private signal of expert 2 is that referendum is canceled or not. In other words, Expert 2 does not hold more information than his private signal, and his announcement is, for a similar reason, 0. In turn, Expert 1 announces 0, as well, and as a result, market fails to aggregate information, because no one learns that Brexit will happen, hence no one learns that the true value of the security is 1 .

On the other hand, if we change appropriately the security of the market, information is aggregated. Consider, the security whose value is 3 when Brexit happens, 2 when No Brexit happens and 1 in case the referendum is canceled. In that case, Expert 1 announcement cannot be the same as in the case that the referendum is canceled. This is true because 1 cannot be given as a convex combination of 3 and 2 . Hence, these two values being different implies that in the next round Expert 2 can infer that Expert's 1 signal cannot be that the referendum is canceled. Combining this piece of information with his own signal, Expert 2 concludes that Brexit should be the realised state, hence 
announcing 1. In the next round, Expert 1 can infer as well that Expert 2 knows that Brexit should happen, therefore information gets aggregated.

In addition, evidence about the difference, on the degree of information aggregation, between a prediction market with and without ambiguity, has been found in the experiment of Chapter 5, with the experiment structure being the same with the example's. In fact, significantly worse predictions have been found for ambiguous markets with the example's Arrow-Debreu security, and no significant differences when we changed it appropriately. ${ }^{4}$

The intuitive difference among the two securities of the example is that the first one is informative for both "subjective expected utility" experts, but not for a "maxmin expected utility" one. This is true because aversion in ambiguity creates, in addition to partial information, an extra layer that precludes information revelation. However, when we increase the diversification of security's values over Expert's 1 information structure, then we can observe that this is a sufficient condition to have the information aggregated.

There is a wide variety of examples, similar to the previous one, in which information does not get aggregated under ambiguity, even if the security is separable under SEU. In particular, the set of beliefs do not have to assign zero probability to some state and the traders do not have to be only two. ${ }^{5}$

The main results of Chapter 2 and Chapter 3 generalise the intuition given in the example. In particular, we define a class of securities, namely separable under ambiguity securities, that aggregate information, under ambiguity, for both myopic and strategic traders (in every converging strategies equilibrium). It turns out that separable under ambiguity securities are separable under $S E U$ and the inclusion is strict. In addition, when the security is not separable under ambiguity, then, for both myopic and strategic traders, information does not get aggregated always.

Finally, our model differs in significant ways from the model of Ostrovsky (2012) and Chen et al. (2012). In the case that traders are myopic, under subjective expected utility assumptions their predictions depend only on the acquired information, however under ambiguity this is not the case. Due to the aversion in ambiguity the current prediction depends on the previous one. In the case that traders are strategic, an equilibrium concept should be defined in order to be compatible with the possible failure of dynamic consistency, whereas this is not needed in the Ostrovsky (2012) and Chen et al. (2012).

\footnotetext{
${ }^{4}$ Another example of the effect of ambiguity in real life prediction markets can be observed in the market opened by Cultivate Labs, trying to predict whether UK will vote leave or remain. The market failed to predict the outcome of the referendum, and its closing prediction was that the probability of voting Brexit is $20 \%$. The market can be found in https://alphacast.cultivateforecasts.com/questions/1311will-the-uk-vote-to-leave-the-eu-in-the-june-2016-referendum.

${ }^{5}$ See in Appendix B for more details.
} 


\subsubsection{Market Microstructure}

There are many approaches to model dynamic markets. The no trade theorems (Milgrom \& Stokey (1982), Sebenius \& Geanakoplos (1983)) imply that in dynamic markets, in which a common prior is assumed, a source to subsidize trading should exist. This is the reason why, on the one hand, the existence of noise traders is usually assumed in models of financial markets and on the other hand heterogeneous priors. In this paper the dynamic market follows the MSR of Hanson (2003) and it is similar to Ostrovsky (2012), Chen et al. (2012) and Dimitrov \& Sami (2008). Whereas the assumption of common prior holds, instead of noise traders there is an automated market maker who admits to have bounded losses. A crucial difference of our setup is that instead of a single common prior, traders hold a common set of multiple priors according to Gilboa \& Schmeidler (1989).

The dynamic trading mechanism in our model starts with an initial public announcement about the value of the security by the market maker, which is made in order to open the market, and with nature choosing, ambiguously, the true state. Then, each trader sequentially announces, in public, their predictions and after every announcement all traders refine their information and beliefs accordingly. A score of each prediction, interpreted as the first part of trader's per period utility, is calculated after the whole uncertainty about the true state is revealed and it is based on a strictly proper scoring rule. In particular, the whole per period utility of a trader is calculated by subtracting from the score of her prediction the score of the prediction made by the previous trader. This can be interpreted as if each time traders make a prediction, they "buy out" the previous one. The scores are derived by a strictly proper scoring rule.

In fact, according to the market mechanism described, and assuming that traders are myopic, it is implied that a trader announces the price of the security that maximises his t-period payoff which is the expected value of the security according to some posterior belief, out of the whole set of beliefs, conditioned to the, possibly refined, information partition. ${ }^{6}$ Therefore, we can conclude that the trading procedure generalises the communication process of Geanakoplos \& Polemarchakis (1982). However, in the MSR model, ambiguity aversion complicates this process considerably, as it implies that each prediction depends on the previous one. Hence, the decision function of each trader is different round by round.

When traders are strategic, the trading procedure is an infinite horizon game with incomplete information. Given that the traders are ambiguity averse, they might potentially be dynamically inconsistent: their optimal continuation strategy at time $t$ might not be optimal at a later time. This is a feature of our model that does not exist in Ostrovsky (2012).

\footnotetext{
${ }^{6}$ That is because the market scoring rule is incentive compatible as a static mechanism for myopic traders.
} 
A widely used equilibrium concept for dynamically inconsistent players is called Consistent Planning, axiomatised in Siniscalchi (2006). According to it, at each time $t$ the corresponding player optimises the continuation payoff over deviations from the current round equilibrium action subject to following the equilibrium afterwards. We use a stronger equilibrium concept than that, called Revision Proof Equilibrium. It was defined in Ales \& Sleet (2014), but here we generalise it for incomplete information. The stronger part of this concept is that it allows the equilibrium strategy to be checked over multiple stage deviations. Intuitively, revision proof equilibrium can be thought as follows: when you are at time $t_{0}$ and considering revising your strategy from that time onwards, you cannot achieve the alternative continuation profile making you better off at every future time $t_{0}+t^{\prime}$, with strictly preference for at least one of them.

\subsubsection{Results}

The results of our theoretical contribution are divided into two Chapters. The results about myopic traders are included in Chapter 2 and the results about strategic traders in Chapter 3.

\subsubsection{Results for myopic traders}

The main result for myopic traders is Theorem 2.7.

It characterises information aggregation in terms of separable under ambiguity securities. In particular, when a separable under ambiguity security is traded, then information gets aggregated in market with myopic trades. Conversely, when a non separable under ambiguity security is traded then there are markets in which information does not get aggregated.

This result is the analogue of Chen et al. (2012) for myopic and ambiguity averse traders, hence completing the literature of information aggregation in that respect.

However, the proof of our theorem indicates an approach of generalising Geanakoplos \& Polemarchakis (1982) in the presence of ambiguity.

More precisely, the proof of our theorem can be separated into two parts. The first one is the part which proves the agreement. The second one is the part that uses the properties of separable under ambiguity securities in order to conclude the information aggregation.

For the first part, we use the convexity of the myopic decision function in order to understand the dynamics of traders' announcements in the evolution of the trading process. Comparing our arguments with the arguments Chen et al. (2012), we do not use the martingale property to conclude the agreement. This is because the martingale 
property is based on the common prior assumption hence is not applicable to our setting. However, we do not use the union consistency either, as it is the case in Dominiak \& Lefort (2015), hence drawing a difference with the existing agreement under ambiguity literature.

For the second part, properties of the separable under ambiguity securities are applied, similarly to Chen et al. (2012). We depart, however, in a substantial way in order to adjust the arguments to the prior by prior updating rule.

\subsubsection{Results for strategic traders}

The main result for strategic traders is Theorem 3.6 and characterises information aggregation in terms of a separable under ambiguity security, dropping the assumption of myopic trades and assuming that traders are stategic.

The theorem, from an intuitive perspective, states that when a separable under ambiguity security is traded in the market and players are in a pure revision proof equilibrium, then information gets aggregated. Conversely, when the security is non separable under ambiguity then there exists a pure revision proof equilibrium at which information does not get aggregated.

This result extends the result of Ostrovsky (2012) when there is ambiguity over the state space. It is the complement of the result with myopic traders, as it allows traders to be strategically large and effect the market with their behavior.

In addition, the strategic result is interesting from a game theoretic point of view. The MSR game is an extensive form game with incomplete information with infinite horizon. The literature does not provide an equilibrium concept, similar to Perfect Bayesian Equilibrium, for such games. We define the Revision Proof equilibrium, Ales \& Sleet (2014), for games of incomplete information and finite horizon.

The proof of the theorem is considerably more complicated and different compared to Ostrovsky (2012). The respective arguments of Ostrovsky (2012) are based on the instant opportunity. In short, this is the action that a myopic trader would play. By having this concept, the proof is following by exploiting heavily the property that there is a common prior, hence the instant opportunity of each trader is independent of the prediction of the previous one.

In our setting such an argument cannot be made. The intuition of our approach can be described in five steps. The first step is to prove that, for every trader, every continuation game, on equilibrium path, is non negative. This is concluded by appropriately defining a deviation strategy which turns out to be a revision that the trader is willing to make, hence we get a contradiction. The second step is to prove that in the limit traders' continuation payoffs are zero. This is proved by using the MSR properties. The third 
step is to prove that in the limit traders are myopic. This is concluded by similar arguments as the first step. Having that conclusion, in the fourth step, we prove that in the limit traders agree. Finally, the fifth step is to use the assumption that the security is separable under ambiguity and hence get the result of information aggregation.

The inverse of the theorem is proved by appropriately defining a revision proof equilibrium. We use the fact that the security is non-separable under ambiguity in order to satisfy the definition of revision proof equilibrium. Then it is shown that information does not get aggregated on equilibrium, hence concluding.

In addition, two other interesting results are given, in particular Theorem 3.7 and Theorem 3.8. These results are in the same spirit as some of the results in Chen et al. (2010) and Dimitrov \& Sami (2008). In particular, we provide some sufficient conditions which imply that traders playing in a revision proof equilibrium are essentially myopic on equilibrium path. In other words, for any player and at any time that she announces a prediction (i.e. information set), assuming no one deviated before, her equilibrium prediction is the same as her myopic prediction at that point.

\subsection{The experimental literature of information aggrega- tion}

In this section of the thesis we will report a part of the literature of experiments on information aggregation. We will analyse and report, mostly, the relevant, to our third chapter, papers.

A large number of experiments have been conducted to measure the efficiency of information aggregation in financial markets and prediction markets. Most of them have studied either double-auctions or parimutuel markets.

In Plott \& Sunder (1988) an experimental double-auction is studied in order to test the theoretical predictions of REE. The results documented in the paper are mixed. In some treatments the markets do not reach REE, whereas in some other they reach the equilibrium.

An explanation of the mixed results in Plott \& Sunder (1988) was given in Forsythe \& Lundholm (1990). The aim of the paper was to give sufficient conditions that are needed to be satisfied in order REE market's model to predict correctly the equilibrium. As it turned out, common knowledge of payoffs along with experience are giving a positive result. That explained the mixed results of Plott \& Sunder (1988), as none of these assumptions was satisfied. 
In Plott et al. (2003) the question of information aggregation is set in the context of a parimutuel market. The results indicate that the more we are moving to more complex environments, the less information gets aggregated. Analysing the weak degree of information aggregation it is concluded that traders are bluffing and they participate in a waiting trading behavior, which both affect negatively the aggregation of information.

Our experimental design is also related to Hanson et al. (2006). This paper documents findings regarding manipulation in prediction markets. The information structure given in that paper is similar to ours. In fact, the private information is given by letting traders know, at each round, which state is not realised.

However our work differs in a main dimension from all these studies. They are using either double auctions or parimutuel markets. On the other hand we use the MSR.

In McKelvey \& Page (1990) the theoretical prediction of McKelvey \& Page (1986b) are tested in a market similar to MSR. In fact, the participants are incentivised by using a proper scoring rule,. This is a communication process very similar to Chen et al. (2012) and Ostrovsky (2012). The results suggest that there is a clear evidence that the information is not pooled in the marker, on the contrary of what theory predicts. However, there is also clear evidence that the public information that is revealed in the market augment each private information.

Finally, another closely related paper is Jian \& Sami (2012). It provides results regarding information aggregation of the MSR of Hanson (2003), under various hypotheses. For example, it is tested whether the trading order, the trading mechanism and the signal distributions affect the efficiency of information aggregation. They test as well if there exist evidence for results like Chen et al. (2012) and Dimitrov \& Sami (2008). In particular, it is tested whether the signal structure is related to myopic or bluffing trading.

\subsection{Our experimental contribution}

In this section we will motivate the third chapter of this thesis, positioning it within the literature of Section 1.3.

\subsubsection{Motivation}

A key question regarding the design of financial markets is whether they have the ability to aggregate and reveal dispersed information. In particular, if information is dispersed among all traders, will their trading behavior lead the market to aggregate information, pricing the securities as if their information was pooled? 
Prediction markets are an example of financial markets created to take advantage of the information aggregation property of securities prices. In these markets the various information pieces of the traders are aggregated in the price, through the buying and selling order of the traders. Hence price is interpreted as an aggregate statistic. In fact, it implicitly implies a prediction with regards to a future event.

The are several ways to implement such a market. Among others, double-auctions and Market Scoring Rules (MSR) are used more and more in practice. Continuous price auctions are well known markets, in terms of format, hence more familiar to traders. However, they are complex for the analyst and the traders to understand its dynamics. However, the MSR are used more and more as an alternative mechanism. Their advantage over the continuous price auction is that they do not depend highly on market liquidity (the market maker admits to have bounded losses) and are more easy to analyse them. In addition, they are similar to double-auctions in terms of format, with the difference that the market maker accepts every bet, without requiring another trader to accept it.

The particular market implementation of MSR can vary. On the one hand, the most intuitive is the probability based market, Hanson (2003), Dimitrov \& Sami (2008) and Chen et al. (2010) among others. Traders just announce directly their beliefs regarding an event. On the other hand it is the security based market Ostrovsky (2012), Chen et al. (2012), Chapter 2, Chapter 3, among others. Similar to the former format, in these markets the predictions regarding the value of a specific security are announced. Our ultimate aim, is to use the controlled environment of the experimental laboratory to draw conclusion with regards to the security based model.

Under the subjective expected utility assumption for the fundamentals of the security Ostrovsky (2012) and Chen et al. (2012) defined the class of separable under SEU securities. It was proved that when such securities are traded in the market, information gets aggregated, even if traders are strategic. By relaxing the assumption of subjective expected utility and allowing for ambiguity aversion in Chapter 2 and Chapter 3 the class of separable under ambiguity securities was defined. It was proved that when such securities are traded in the market then information gets aggregated. It was proved, as well, that it is not, in general, true, in a setting with ambiguity, that with separable under SEU securities information gets aggregated.

In fact, Arrow-Debreu securities, which belong to the class of separable under $S E U$ ones, do not always aggregate information in a setting with ambiguity, as opposed to the case of subjective expected utility. However, a modification of an Arrow-Debreu security can aggregate information either with or without the presence of ambiguity.

The main scope of this paper is to experimentally test these theoretical predictions. In particular, to test the hypothesis that ambiguity affects the degree of information 
aggregation for a separable under SEU security but not for a separable under ambiguity security.

\subsubsection{Results}

Two main hypothesis tests are conducted. These tests aim to test the theoretical prediction of the two first chapters the thesis.

Firstly, we are testing the difference, in terms of information aggregation, between a market without ambiguity and a market with ambiguity when a separable under SEU security is traded.

Secondly, we are undertaking a similar test but replacing the separable under SEU security with a separable under ambiguity one.

Chapter 5 concludes in favor of our theoretical results. In particular, as far as the first hypothesis test is concerned, evidence was given that the degree of information aggregation is significantly worse in the market with ambiguity. However, when replacing the separable under SEU security with a separable under ambiguity one evidence was given that the degree of information aggregation is indistinguishable statistically.

Most results are significant at 5\%, hence giving a fairly strong evidence in favor of the corresponding theoretical results. 


\section{Chapter 2}

\section{Information Aggregation with Myopic Traders}

This paper studies information aggregation in a dynamic trading model under ambiguity with finite, partially informed and ambiguity averse traders. Instead of the commonly used assumption of common prior, here we assume that traders share a common set of multiple priors. By defining the class of separable under ambiguity securities, we show that they characterise information aggregation for every set of priors that myopic traders share. Comparing to the class of separable under SEU securities, which provide a similar characterisation with the standard subjective expected utility assumptions, our class is more restrictive and in particular it is included strictly in the class of separable under SEU securities. In fact, Arrow-Debreu securities do not belong to the class of separable under ambiguity securities, whereas they are separable under SEU. Hence it is concluded that information aggregation is not always possible when myopic traders are ambiguity averse, even if information aggregation is the case under subjective expected utility assumptions.

\section{$2.1 \quad$ Introduction}

A key question regarding the design of financial markets is whether they have the ability to aggregate and reveal dispersed information. In particular, if information is dispersed among all traders, will their trading behavior lead the market to aggregate information, pricing the securities as if their information was pooled?

Having traders fully informed about the price of a security would imply that whenever the security is mispriced, they would buy or sell it accordingly and hence its price would move towards the correct one. However, traders are not always fully informed about the true prices of the securities. In contrast, they are rather partially informed and, even 
worse, the complexity of the financial system and the inherent post crisis uncertainty makes traders ambiguous regarding the true values of the securities.

In other words, traders in many cases believe that the price of a security in the future will be a certain amount, but the probability that they give for the correct accuracy of this prediction is a whole set of probabilities $\mathcal{P}$ and not unique. For example, consider a firm investing on a project, whose cash flows depend on the future temperature levels, and a derivative security paying off in case the price of firm's stock will go up until a certain date in the future and paying nothing in case it will go down. However, the phenomenon of global warming cannot be compared with similar phenomena observed in previous centuries. Hence the cycles of the temperature differ significantly from those observed in the past. Therefore, one could argue that traders, in addition to their partial information, cannot quantify the event under consideration with a unique probability and hence they hold ambiguous beliefs about the true price of the stock. ${ }^{7}$

In particular, this can be of substantial importance in markets where an asset about a future uncertain event is traded, as described above. Prediction markets are an example of such markets. In prediction markets securities, contingent to a particular future event, are bought and sold in order to aggregate traders' information. The type of securities range from securities regarding financial events to securities regarding geopolitical and sport events. As the previous example illustrated, and because of the nature of these markets, the events on which the securities depend are of high uncertainty by themselves: events about terrorist attacks, geopolitical issues, technological innovations and environmental issues along with the events about financial issues are inherently ambiguous.

Many firms have organised internal prediction markets. Google, Ford and General Electric, HP among others run internal prediction markets as a corporate governance and predictions tool. In addition, Inkling Markets, Consensus Point, Crowdcast are some of the public prediction markets.

Therefore, a number of interesting questions arise. What happens in terms of information aggregation for such markets for ambiguous events? Do the prices of the securities get priced as if all traders' information was pooled? Does the aggregation depend on the type of the security? What new restrictions does ambiguity create to the securities, compared to the subjective expected utility assumptions, in order information aggregation to be guaranteed no matter what traders' beliefs are? The main focus of this paper is to study and answer these questions for a fairly general class of dynamic markets by defining the class of securities that aggregate information for myopic traders, relaxing the assumption of subjective expected utility and allowing for ambiguity aversion. In the standard model with subjective expected utility and common prior, the class of securities that achieve

\footnotetext{
${ }^{7}$ More formally, we could equivalently assume, instead, that because of the huge number of variables that affect the direction of the stock price and cannot be observed, the probability over the states is ambiguously determined by a multidimensional Ellsberg urn.
} 
full information aggregation has been characterised, both in the strategic (Ostrovsky (2012)) and in the non-strategic case (Chen et al. (2012)). Such securities are called separable under SEU.

The contribution of this paper is therefore threefold. Firstly, assuming ambiguity means that many useful properties that are inherent to subjective expected utility, like dynamic consistency, value of information and unique prior, do not hold. The paper studies information aggregation in MSR markets when such properties do not hold. The solution is given by defining a particular class of "well behaved" securities, namely the separable under ambiguity securities, and showing that these securities aggregate information for any set of priors. Secondly, we show that the class of separable under ambiguity securities is strictly contained in the class of separable under SEU ones, hence implying that aggregation of information is harder under ambiguity. In fact, among several examples of separable under SEU securities that do not aggregate information are examples in which Arrow-Debreu securities do not aggregate information. Finally, the MSR within subjective expected utility assumptions is essentially the, so called, opinion game and in Geanakoplos \& Polemarchakis (1982) it is proven that in the opinion game finally the agents agree. We provide here the same result for the opinion game under ambiguity.

There are many approaches to model dynamic markets. The no trade theorems (Milgrom \& Stokey (1982), Sebenius \& Geanakoplos (1983)) imply that in dynamic markets, in which a common prior is assumed, it should exist a source to subsidize trading. This is the reason why, on the one hand the existence of noise traders is usually assumed in models of financial markets and on the other hand heterogeneous priors. In this paper the dynamic market follows the Market Scoring Rule (MSR) of Hanson (2003) and it is similar to Ostrovsky (2012) and Dimitrov \& Sami (2008). Instead of noise traders there is an automated market maker who admits to have losses. The only difference of our setup is that instead of a single common prior, traders hold a common set of multiple priors according to Gilboa \& Schmeidler (1989).

The dynamic trading mechanism in our model starts with an initial public announcement about the value of the security by the market maker, which is done in order to open the market, and with nature choosing, ambiguously, the true state. Then, each trader sequentially announces, in public, their predictions and after every announcement all traders refine their information and beliefs accordingly. The score of each prediction, interpreted as, the first component of, trader's per period utility, is calculated after the whole uncertainty about the true state is revealed and it is based on a strictly proper scoring rule. In particular, the whole per period utility of a trader is calculated by subtracting from the score of her prediction the score of the prediction made by the previous trader. This can be interpreted as if each time traders make a prediction, they "buy out" the previous one. 
In fact, according to the market mechanism as described, and assuming that traders are myopic it is implied that a trader announces the price of the security that maximises his t-period payoff which is the expected value of the security according to some posterior belief, out of the whole set of beliefs, conditioned to the, possibly refined, information partition. ${ }^{8}$ Therefore, we can conclude that the trading procedure generalises the communication process of Geanakoplos \& Polemarchakis (1982) by allowing traders to hold many beliefs at each round, due to ambiguity. However, ambiguity aversion complicates this process considerably, as it implies that each prediction depends on the previous one. That means, the decision function of each trader is different and also is, potentially, sensitive to other market specific components. Because of the trader-and-market specific utility function traders can in principle agree to disagree and not have the same finest information set that determine their utilities in the sense of Geanakoplos (1995). ${ }^{9}$ However, at the end, if the announcement of each trader converge to some real number, then the traders cannot disagree forever.

A natural question that comes out, is why one would want to study information aggregation in MSR type markets under ambiguity? Firstly, the reason is that these type of markets are becoming more and more popular in real life prediction markets, many of which we mentioned before. Secondly, even if it appears to lack the process under which securities are bought and sold, essentially the MSR can be reinterpreted in order to correspond to the classic approach with an automated market maker who continuously adjusts the price of the securities as traders buy from and sell to him. ${ }^{10}$ Thirdly, as it was mentioned before as one of the main intuitions that this paper contributes to the existing literature, the Arrow-Debreu securities does not always aggregate information when the traders are ambiguity averse. However, Arrow-Debreu securities, and other similar simple securities, are very popular and convenient to use in prediction markets designed for corporate governance and predictions. Hence, ambiguity might substantially and practically complex or even fail such markets when ambiguity averse traders are participating.

\subsubsection{Literature Review}

A lot of work has been evolved related to information aggregation and information revelation in dynamic markets, with Hayek (1945) to begin with. Grossman (1976) proved that in equilibrium the price aggregates information. Radner (1979) introduced the concept of Rational Expectations Equilibrium (REE) and proved that generically the prices aggregate information dispersed among traders (fully revealing REE). Several

\footnotetext{
${ }^{8}$ That is because the market scoring rule is incentive compatible as a static mechanism for myopic traders.

${ }^{9}$ However, if we assume the union consistency property for the decisions function we can conclude that the common information set across traders exists, and we can prove the agreement as well. See the literature review part for more discussion.

${ }^{10}$ See in Appendix C for more details.
} 
results have been proven regarding the convergence in REE in dynamic settings: Hellwig (1982), Dubey et al. (1987), Wolinsky (1990), Golosov et al. (2014), McKelvey \& Page (1986a), Nielsen et al. (1990), Nielsen (1984) among others.

In Aumann (1976), Geanakoplos \& Polemarchakis (1982), Cave (1983), Sebenius \& Geanakoplos (1983), Nielsen (1984), Nielsen et al. (1990) information communication is studied either in an opinion game and a trading procedure for posterior beliefs or other aggregate statistics. These papers however do not fully characterise under what conditions the consensus yields the true posterior or expectation of the security. DeMarzo \& Skiadas (1998), DeMarzo \& Skiadas (1999) go beyond the consensus result of the former papers finding necessary and sufficient condition for information aggregation. Furthermore, Chen et al. (2012), Ostrovsky (2012) are dealing with information aggregation of myopic and strategic traders, under a similar assumption for the securities as DeMarzo \& Skiadas (1998), DeMarzo \& Skiadas (1999), and prove that this assumption is both necessary and sufficient for information aggregation either for a single security or when many securities are traded. In Chen et al. (2012), Ostrovsky (2012) the models are based on MSR ${ }^{11}$ and thus their results, even fairly general, are particularly related to prediction markets as well. Similar approaches can be found in Chen et al. (2010), Dimitrov \& Sami (2008) where the focus is on considering undiscounted games based on a particular scoring rule, the logarithmic.

In addition, there are several results attempting to study the effect of ambiguity on information revelation. In particular, Dominiak \& Lefort (2015), Dominiak \& Lefort (2013), Carvajal \& Correia-da Silva (2010), Kajii \& Ui (2005) and Kajii \& Ui (2009), among others, are related mostly to the seminal cannot agreeing on disagree type result of Aumann (1976). In fact, they study a consensus result in the presence of ambiguity averse traders (Kajii \& Ui (2005), Kajii \& Ui (2009)) and Carvajal \& Correia-da Silva (2010) or with CEU and neo-additive capacities (Dominiak \& Lefort (2015), Dominiak \& Lefort (2013)). Finally, within a REE setting expanded in order to include preferences that display ambiguity aversion, the existence and robustness of partially-revealing rational expectations equilibria (REE) is proved in Condie \& Ganguli (2011).

Our contribution on the literature is, therefore, twofold. On the one hand, our result for myopic trades contributes to the literature of the effect of ambiguity on information revelation. In fact, we prove that myopic, partially informed ambiguity averse traders with a common set of priors cannot disagree forever in an MSR market. On the other hand we contribute to the literature of information aggregation by defining a class of securities which characterises information aggregation, for both myopic and strategic traders, in the presence of ambiguity.

\footnotetext{
${ }^{11}$ For Ostrovsky (2012) that is true only for the first model.
} 


\subsubsection{Overview}

This paper is organized as follows. The next section describes the model. In particular, in Section 2.1.3 we describe the standard MSR trading model which is the first building block of our model, in Section 2.1.4 we proceed with the details regarding the particular ambiguity model and in Section 2.1.5 we describe how ambiguity is applied in the trading model, and in particular we discuss the decision function for myopic traders. In Section 2.2 the notion of information aggregation is defined and in Section 2.3 we define the class of separable under ambiguity securities. In Section 2.4 the main result is mentioned. Finally we conclude in the last section. All proofs are included in Appendix A.

\subsubsection{Trading Environment}

The state space is assumed to be finite, denoted as $\Omega=\left\{\omega_{1}, \ldots, \omega_{l}\right\}$. We assume the powerset $\mathbf{P}(\Omega)$ to be the sigma-algebra over the state space. We define the order with respect to which the finite number of traders will participate in the market, call it $\mathcal{O}$, as the one to one and onto function $\mathcal{O}:\{\mathcal{T}\} \longrightarrow\{1, \ldots, n\}$, from the set of traders to the set $\{1, \ldots, n\}$. Denote the information structure under ordering $\mathcal{O}$ by $\Pi_{1}, \ldots, \Pi_{n}$, hence the $\Pi_{i}$ partition is of the $\mathcal{O}^{-1}(i)$ trader, assuming that the join of all agents' partitions to be singleton. The security is defined as a measurable function $X: \Omega \longrightarrow \mathbb{R}$ and the traders are risk neutral. We restrict attention, from here and onwards, to continuous strictly proper scoring rules $s$ and we assume that all predictions belong to the set $Y=\left[\min _{\omega \in \Omega} X(\omega), \max _{\omega \in \Omega} X(\omega)\right]$.

With the MSR, the trading starts with the market maker's (MM) initial public announcement, about the real value of the security. Then, the first trader makes his own prediction, which is, in turn, publicly announced. Similarly, the second player, who observes the previous prediction, further modifies it by making his own, and so on until the last player, after which the first player can again modify the prediction, and the whole cycle repeats an infinite number of times. ${ }^{12}$

The infinite number of trading rounds does not mean that the trade never ends. Following Ostrovsky (2012), the infinite time periods that the market is open is, in fact, a discretisation of the interval $[0,1]$ i.e. $0<t_{1}<\ldots<t_{n}<\ldots<t_{\infty}=1$, where in period $t_{\infty}$ the real value of the security is revealed and each prediction is evaluated according to the strictly proper scoring rule s. More formally, a proper scoring rule is defined as a function $s(y, X(\omega))$, with $X(\omega)$ being the value of the random variable $X$ when the state is $\omega$ and $y$ being the prediction. In addition, if there is a common

\footnotetext{
${ }^{12}$ As it will be formally more clear in Section 2.1.5, we use the notation for the order, because the MSR is a sequential market, and ambiguity makes sensitive each prediction to the prediction of the previous trader. Hence by using such a notation we make our results robust against that sensitivity.
} 
prior $p$, for every random variable $X$ the expectation of $s$ over the state space is maximised for $y=E_{p}[X]$. If the maximising value is unique, then it is called strictly proper scoring rule. Examples of strictly proper scoring rules that are, in addition, continuous are the quadratic and the logarithmic scoring rule, given by $s(y, x)=-(x-y)^{2}$ and $s(y, x)=(x-a) \ln (y-a)+(b-x) \ln (b-y)$ with $a<\min _{\omega \in \Omega} X(\omega), b>\max _{\omega \in \Omega} X(\omega)$, respectively, (Ostrovsky (2012)).

In particular, assume that at some time $t^{*}>1$ the true value $X\left(\omega^{*}\right)$ is revealed. Then, the payoff of the trader from changing the prediction from $y_{t-1}$ to $y_{t}$, is of the form $\beta^{t}\left(s\left(y_{t}, X\left(\omega^{*}\right)\right)-s\left(y_{t-1}, X\left(\omega^{*}\right)\right)\right)$, with $\beta \in(0,1)$. Reinterpreting it, the player "buys out" the previous player's prediction.

The setting, as described, is assumed common knowledge among the traders.

\subsubsection{Ambiguity}

While in the previous section we illustrated the general trading environment, which is similar to the subjective expected utility case, here we give details about our main feature of the model, which is introducing ambiguity.

A widely used way to model subjective uncertainty in economics is to use the theory of subjective expected utility. Intuitively, an implication of such a perspective is that the decision maker quantify with a single probability the uncertain event under consideration. However, if the decision maker has imprecise knowledge of the uncertainty of the event then his beliefs are better represented by a set of probabilities rather than a unique one. An ambiguity averse decision maker adjusts his choice on the side of pessimism or optimism in response to his imprecise knowledge of the uncertainty.

In this thesis agents are assumed to have ambiguity averse preferences according to Gilboa \& Schmeidler (1989) model. Therefore we assume the set of multiple priors to be a convex and closed subset $\mathcal{P} \subseteq \Delta(\Omega)$, common among traders, and endowed with the weak* topology on $\Delta(\Omega)$ (with $\sigma$-algebra as specified before). Intuitively, under that definition of ambiguity traders are having a more uncertainty that a single probability could quantify and they optimise their behavior by considering the worst scenario under the set of priors.

For any set of priors $\mathcal{Q}$ over any finite state space $S$, we define $\bigcup_{q \in \mathcal{Q}} S u p p(q)=\{s \in S$ : $q(s)>0$ for some $q \in \mathcal{Q}\}$.

Given that, in our setting we consider as states that are possible to be realised the states of $\Omega$ for which there is assigned strictly positive probability for some $p \in \mathcal{P}$. Formally, this is the subset $\{\omega \in \Omega: p(\omega)>0$ for some $p \in \mathcal{P}\}=\bigcup_{p \in \mathcal{P}} \operatorname{Supp}(p)$. 


\subsubsection{Myopic Traders}

This part is about defining formally the optimisation problems that myopic traders face. In particular, we give details about what we mean by myopic traders and analyse their decision function.

Let a compact and convex set of priors $\mathcal{P}$ over the state space $\Omega$, and $\omega^{*}$, for which there exists $p \in \mathcal{P}$ with $p\left(\omega^{\star}\right)>0$, to be the realised state. Consider a player $i$ who trades at periods $t_{i+n k}$, with $k \in \mathbb{N}$, given a strictly proper scoring rule $s$, MM's initial announcement $y_{0}$ and an ordering $\mathcal{O}$. A myopic strategy of the player is a sequence $y^{i}=\left(y_{i+n k}\right)_{k \in \mathbb{N}}$, where $y_{i+n k}: \Pi_{i} \times Y \longrightarrow Y$ is a measurable function, for every $k \in \mathbb{N}$.

Define the set

$$
\mathcal{F}_{1}\left(\omega^{*}, y_{0}\right)=\left\{\omega^{\prime} \in \mathcal{F}_{0}\left(\omega^{*}\right): y_{1}\left(\Pi_{1}\left(\omega^{\prime}\right), y_{0}\right)=y_{1}\left(\Pi_{1}\left(\omega^{*}\right), y_{0}\right)\right\}
$$

where $\mathcal{F}_{0}\left(\omega^{*}\right)=\{\omega \in \Omega: p(\omega)>0$ for some $p \in \mathcal{P}\}$. This set can be interpreted as the information available to a fictitious observer who holds no private information, after the first prediction $y_{1}$. Hence every trader $i$ can refine her own information as $\mathcal{F}_{1}\left(\omega^{*}, y_{0}\right) \cap \Pi_{i}\left(\omega^{*}\right)$.

Then $y_{2}$ becomes now public and the whole process is repeated for every time step t, with traders taking turns revising their predictions.

Formalising the previous reasoning, the public information, when changing the prediction from $y_{i+n k}$ to $y_{i+1+n k}$, for every $i$, every state $\omega^{*}$ that can be realised and every round $k \in \mathbb{N}$, is given by

$\mathcal{F}_{i+1+n k}\left(\omega^{*}, y_{i+n k}\right)=\left\{\omega^{\prime} \in \mathcal{F}_{i+n k}\left(\omega^{*}\right): y_{i+1+n k}\left(\Pi_{i+1}\left(\omega^{\prime}\right), y_{i+n k}\right)=y_{i+1+n k}\left(\Pi_{i+1}\left(\omega^{*}\right), y_{i+n k}\right)\right\}$

and the corresponding private information for trader $i+2$ is $\mathcal{F}_{i+1+n k}\left(\omega^{*}, y_{i+n k}\right) \cap$ $\Pi_{i+2}\left(\omega^{*}\right)$.

We define a sequence of beliefs $\left(\mathcal{P}_{i+k n}\right)_{k \in \mathbb{N}}$, with $\mathcal{P}_{0}=\mathcal{P}$, as the beliefs of $i$ at round $k$.

Definition 2.1. We define $\left(y,\left(\mathcal{P}_{i+k n}\right)_{i \in\{1, \ldots, n\}, k \in \mathbb{N}}\right), y=\left(y_{1}, \ldots, y_{n}\right)$, to be a myopic equilibrium if for every $i=1, \ldots, n$ and every $k \in \mathbb{N}$ :

(i) $y_{i+n k}\left(\Pi_{i}(\omega), y_{i-1+n k}\right)=d_{\mathcal{P}}\left(\Pi_{i}(\omega) \cap \mathcal{F}_{i-1+n k}\left(\omega, y_{i-2+n k}\right), y_{i-1+n k}\right)=$ $\underset{y \in Y}{\operatorname{argmaxmin}} E_{p \in \mathcal{P}} E_{p_{\mid \Pi_{i}(\omega) \cap \mathcal{F}_{i-1+n k}\left(\omega, y_{i-2+n k}\right)}}\left[s(y, X)-s\left(y_{i-1+n k}, X\right)\right]$ for every $\omega$ with $p(\omega)>0$ for some $p \in \mathcal{P}$.

(ii) For every $\omega \in \Omega$ and for every $i=1, \ldots, n$ : there exists $p^{\prime} \in \mathcal{P}$ with $p^{\prime}\left(\Pi_{i}(\omega) \cap\right.$ $\mathcal{F}_{i-1+n k}\left(\omega, y_{i-2+n k}\right)>0$ if and only if for every $p \in \mathcal{P}$ it is $p\left(\Pi_{i}(\omega) \cap \mathcal{F}_{i-1+n k}\left(\omega, y_{i-2+n k}\right)\right)>$ 0 and $i$ updates every prior using Bayes rule. 
We can observe that Definition 2.1 (ii), implies that the beliefs of the traders at a myopic equilibrium are given by prior by prior updating.

Definition 2.2. A player $i$ is myopic, if for every $k \in \mathbb{N}$ it holds that $y_{i+n k}\left(\Pi_{i}\left(\omega^{*}, y_{i-1}\right)=\right.$ $d_{\mathcal{P}}\left(\Pi_{i}\left(\omega^{*}\right) \cap \mathcal{F}_{i+n k}\left(\omega^{*}, y_{i-1+n k}\right), y_{i+n k}\right)$, by using prior by prior updating.

From now on, when we will say that traders are myopic on a specific path or for some specific prediction, it will be shown (or will be obvious) that prior by prior updating is applicable wherever is needed.

In addition, in order to keep notation as simple as possible, we say that all players are myopic, we mean that they are in a myopic equilibrium. The details of the myopic equilibrium will be obvious form the context or explicitly specified, otherwise.

Consider a security and an information structure of the traders. Given a strictly proper scoring rule $s$, an initial announcement of the MM $y_{0}$ and an ordering of traders $\mathcal{O}$, we define the set $\mathcal{A}_{s, y_{0}, \mathcal{O}}=\left\{\mathcal{P} \subseteq \Delta(\Omega):\left(y,\left(\mathcal{P}_{i+k n}\right)_{i \in\{1, \ldots, n\}, k \in \mathbb{N}}\right)\right.$ is a myopic equilibrium $\}$. We call that property regularity. ${ }^{13}$

We need to prove that the function $d_{\mathcal{P}}$ of Definition $2.1(i)$ is, indeed, well defined. We provide below Lemma 2.3 which shows, firstly, that this is a function and, secondly, it provides its general functional form. Note that for $\mathcal{P}=\{p\}$ we get exactly the decision function in the subjective expected utility case (i.e. Ostrovsky (2012) and Chen et al. $(2012)) \cdot{ }^{14,15}$

Lemma 2.3. Let $s$ be a continuous strictly proper scoring rule on $Y=[a, b], a, b \in \mathbb{R}$. Then, $\operatorname{argmax}_{y \in Y} \min _{p \in \mathcal{P}} E_{p}[s(y, X)-s(z, X)]$ exists, is a singleton and is equal to the expectation of $X$ with respect to some $p \in \operatorname{argmin}_{p \in \mathcal{P}} \max _{y \in Y} E_{p}[s(y, X)-s(z, X)]$ (which might not be singleton). In particular, the unique solution to the optimisation problem is of the form $y=E_{p}[X]$, for some $p \in \mathcal{P}$.

The proof of the Lemma 2.3 can be found in Appendix A.

It is important to highlight that the dependence of the decision function in the previous announcement, makes the myopic behavior more complex than myopic behavior under SEU. In particular, the myopic behavior of the previous affects the one of the next player.

\footnotetext{
${ }^{13}$ We can observe that not for any set of priors a myopic equilibrium exists. For example take the quadratic scoring rule, the information partition $P_{1}=\left\{\left\{\omega_{1}, \omega_{2}\right\},\left\{\omega_{3}\right\}\right\}$ and $P_{2}=\left\{\left\{\omega_{1}, \omega_{3}\right\},\left\{\omega_{2}\right\}\right\}$, the security $X\left(\omega_{1}\right)=X\left(\omega_{2}\right)=1, X\left(\omega_{3}\right)=0$, the initial announcement to be $y_{0}=0$ and the set of priors $\mathcal{P}=\operatorname{conv}\left\{p^{1}, p^{2}\right\}$ with $p^{1}=\left(0, \frac{1}{3}, \frac{1}{3}, \frac{1}{3}\right), p^{2}=\left(\frac{1}{4}, \frac{1}{4}, \frac{1}{4}, \frac{1}{4}\right)$.

${ }^{14}$ Lemma 2.3 is related to a result in Chambers (2008). The proofs are closely related, too.

${ }^{15}$ It is straightforward to observe that for $\mathcal{Q}=\left\{q=p\left(. \mid \Pi_{i}(\omega) \cap A\right): p \in \mathcal{P}\right\}$ it is $d_{\mathcal{P}}\left(\Pi_{i}(\omega) \cap A, z\right)=$ $d_{\mathcal{Q}}(\Omega, z)=\underset{y \in Y}{\arg \operatorname{maxmin}_{q \in \mathcal{Q}}} E_{q}[s(y, X)-s(z, X)]$, where $A \subseteq \Omega$. This explains why Lemma 2.3 refers to the myopic decision function in the dynamic setting.
} 


\subsection{Information Aggregation}

In this part of the paper we formally define the concept of information aggregation. In addition we compare it with the respective definition given in Ostrovsky (2012) and Chen et al. (2012).

Definition 2.4. Information gets aggregated with respect to security $X$, information partition $\Pi$, strictly proper scoring rule $s$, initial announcement $y_{0}$, order of traders $\mathcal{O}$, and $\mathcal{P} \in \mathcal{A}_{s, y_{0}, \mathcal{O}}$ if the sequence of myopic predictions $y_{1}, \ldots, y_{k}, \ldots$ converges to the true value of the security, and the set of states that this does not hold is of zero probability for all priors in $\mathcal{P}$.

The definitions that were provided in Chen et al. (2012) and Ostrovsky (2012) were similarly stated. However, their definition tries to accommodate mixed strategies, which is not the case with our definition. In addition, in our setting we have a compact and convex set of priors that, in general, is not singleton. That is the reason why we should impose the regularity property as well.

\subsection{Separability Under Ambiguity}

The topic of this section is to give the properties needed to be satisfied by the securities in order to be able to characterise information aggregation for every set of priors. The condition is motivated by the example below.

The notion of separability under SEU, as given under subjective expected utility assumptions, Chen et al. (2012) and Ostrovsky (2012), does fit in naturally, in our setting. In particular, their definition takes the following form:

Definition 2.5. A security $X$ is called non-separable under SEU under partition structure $\Pi$ if there exists $p$ and $v$ such that:

(i) $X$ is not constant at $\{\omega \in \Omega: p(\omega)>0\}$.

(ii) For every $i=1, \ldots, n$ and $\omega$, with $p(\omega)>0$, it is $E_{p}\left[X \mid \Pi_{i}(\omega)\right]=v$.

We can observe that separability under SEU is a property given the security and the information structure. Hence has nothing to do with ambiguity aversion per se.

However, this concept of separable under SEU securities is not enough to guarantee information aggregation for every set of priors for ambiguity averse traders, when all traders are myopic, as the following example shows. 


\section{Example:}

Consider two traders, their information partitions $\Pi_{1}=\left\{\left\{\omega_{1}, \omega_{2}\right\},\left\{\omega_{3}, \omega_{4}\right\}\right\}, \Pi_{2}=$ $\left\{\left\{\omega_{1}, \omega_{3}\right\},\left\{\omega_{2}, \omega_{4}\right\}\right\}$ and the common set of priors to be $\mathcal{P}=\operatorname{conv}\left\{p^{1}, p^{2}\right\}$ with $p^{1}=$ $\left(0, \frac{1}{3}, \frac{1}{3}, \frac{1}{3}\right), p^{2}=\left(\frac{1}{4}, \frac{1}{4}, \frac{1}{4}, \frac{1}{4}\right)$. In addition we assume an Arrow-Debreu security with $X\left(\omega_{1}\right)=1, X\left(\omega_{2}\right)=X\left(\omega_{3}\right)=X\left(\omega_{4}\right)=0$ and we consider the quadratic scoring rule which gives to a prediction $y$, given the realisation of the random variable is $x^{*}$, the score $s\left(y, x^{*}\right)=-\left(y-x^{*}\right)^{2}$.

Let the initial price of the security is $y_{0}=0$, set by the market maker. Assuming the realised state to be $\omega_{1}$, the correct price to be inferred is $X\left(\omega_{1}\right)=1$. Trader 1 , having received a private signal according to its information partition, maximises myopically, her utility. In fact, $\min _{p \in \mathcal{P}} E_{p \mid\left\{\omega_{1}, \omega_{2}\right\}}\left[s\left(E_{p \mid\left\{\omega_{1}, \omega_{2}\right\}}[X], X(\omega)\right)-s(0, X(\omega))\right]=$ $\min _{p \in \mathcal{P}}\left[\left(\frac{p\left(\omega_{1}\right)}{p\left(\left\{\omega_{1}, \omega_{2}\right\}\right)}\right)^{2}\left(2-\frac{p\left(\omega_{1}\right)}{p\left(\left\{\omega_{1}, \omega_{2}\right\}\right)}-\frac{p\left(\omega_{2}\right)}{p\left(\left\{\omega_{1}, \omega_{2}\right\}\right)}\right)\right]=\min _{p \in \mathcal{P}}\left(\frac{p\left(\omega_{1}\right)}{p\left(\left\{\omega_{1}, \omega_{2}\right\}\right)}\right)^{2}$. We then conclude that the solution is a probability measure that gives $p\left(\omega_{1}\right)=0$. Therefore, the prediction of the trader 1 would be $y_{1}=0$. We can observe that this would be the prediction of trader 1 in case her private signal was $\left\{\omega_{3}, \omega_{4}\right\}$, too. ${ }^{16}$

From trader's 2 point of view, she does not get more information than her private signal itself. In particular, her reasoning is as follows: if trader 1 was at $\left\{\omega_{1}, \omega_{2}\right\}$ she indeed would give $y_{1}=0$. If, on the other hand, trader 1 was at $\left\{\omega_{3}, \omega_{4}\right\}$ then she would, as well, give $y_{1}=0$. Therefore, we can conclude that trader 2 cannot obtain a finer partition than her private signal $\left\{\omega_{1}, \omega_{3}\right\}$.

Based on that, trader 2 maximises, myopically, her utility. Similarly to trader 1 , it is $\min _{p \in \mathcal{P}} E_{p \mid\left\{\omega_{1}, \omega_{3}\right\}}\left[s\left(E_{p \mid\left\{\omega_{1}, \omega_{3}\right\}}[X], X(\omega)\right)-s(0, X(\omega))\right]=\min _{p \in \mathcal{P}}\left[\left(\frac{p\left(\omega_{1}\right)}{p\left(\left\{\omega_{1}, \omega_{3}\right\}\right)}\right)^{2}\left(2-\frac{p\left(\omega_{1}\right)}{p\left(\left\{\omega_{1}, \omega_{3}\right\}\right)}-\right.\right.$ $\left.\left.\frac{p\left(\omega_{3}\right)}{p\left(\left\{\omega_{1}, \omega_{3}\right\}\right)}\right)\right]=\min _{p \in \mathcal{P}}\left(\frac{p\left(\omega_{1}\right)}{p\left(\left\{\omega_{1}, \omega_{3}\right\}\right)}\right)^{2}$. The solution is again , therefore, a probability measure with $p\left(\omega_{1}\right)=0$. Hence trader's 2 prediction is $y_{2}=0$.

After the announcement of $y_{2}$, trader 1 would reason that either trader 2 being at $\left\{\omega_{1}, \omega_{3}\right\}$ or $\left\{\omega_{2}, \omega_{4}\right\}$, she would predict $y_{2}=0$.

Finally, no trader strictly refines her initial signal at any round (which implies as well that the regularity assumption is satisfied), therefore everyone keeps saying 0 at any round, but the true value is 1 and hence there is no information aggregation, although they could aggregate their information, by pooling their information partitions at state $A$ and realise the true price. ${ }^{17}$

In fact, the expression "no trader strictly refines her initial signal at any round" means that both keep believing that both states of their private information can be realised

\footnotetext{
${ }^{16}$ The argument is based on Lemma 2.3. According to it, if we find a solution $p^{*}$ to the "minmax", then we get the solution of the "maxmin" optimisation problem as $y^{*}=E_{p^{*}}[X]$.

${ }^{17}$ In Appendix B there are various examples of separable under SEU securities that are not separable under ambiguity.
} 
(they keep believing that because there is at least one probability that assigns to both states strictly positive likelihood).

In particular, this example illustrates that an Arrow-Debreu security might fail to aggregate information for ambiguity averse myopic traders. In addition, although the details are part of the following chapter, the myopic equilibrium in the previous example is a strategic equilibrium, hence concluding that in a market with an Arrow-Debreu security information does not get aggregated even if traders are strategic. ${ }^{18}$

Therefore, a different concept of ambiguity-related separability property is needed. Indeed, we suggest a strengthening of the concept of separable under SEU which turns out to be appropriate to characterise information aggregation for every set of priors for the dynamic market we described.

In fact, the new notion of separability that we suggest tries to leave out of its class all the securities that does not reveal information when some trader is more informed, as in the example before.

We follow Ostrovsky (2012) and define non-separability under ambiguity, as its definition can be interpreted more easily than the definition of separability under ambiguity.

Definition 2.6. A security $X$ is called non-separable under ambiguity under partition structure $\Pi$ and proper scoring rule $s$ if there exists an ordering of traders $\mathcal{O}$, a set $\mathcal{P} \subseteq \Delta(\Omega)$, which is convex compact and mutually absolute continuous with respect to $\Pi_{i}$ for every $i=1, \ldots, n^{19}$, and $v$ such that:

(i) $X$ is not constant at $\{\omega \in \Omega$ : there exists $p \in \mathcal{P}$ with $p(\omega)>0\}$

(ii) For every $i=1, \ldots, n$ it is $d_{\mathcal{P}}\left(\Pi_{i}(\omega), v\right)=v$ for every $\omega$, such that there exists $p \in \mathcal{P}$ with $p(\omega)>0$.

A way to interpret the concept of separability under ambiguity is thinking of it as the property that allows in its class only those non-informative securities that turn out to be the trivial ones at $\bigcup_{p \in \mathcal{P}} \operatorname{Supp}(p)$, in case no information is communicated. Relating the definition to the previous example, we can observe that the provided Arrow-Debreu security is not separable under ambiguity under the given information partition structure and quadratic proper scoring rule, as long as for the priors and ordering given in the example, and for $v=0$, condition ( $i i)$ in the definition is satisfied for all states, but

\footnotetext{
${ }^{18}$ When we are saying here strategic equilibrium, we mean the revision proof equilibrium of Definition 3.3. The arguments to conclude that it is indeed a revision proof equilibrium are the same as given in the proof of Theorem 3.6 (ii), in Appendix B, for $v=0$.

${ }^{19}$ We define it as follows: for every $i=1, \ldots, n$ and every $\omega \in \Omega$ it holds that, there exists $p^{\prime} \in \mathcal{P}$ with $p^{\prime}\left(\Pi_{i}(\omega)\right)>0$ if and only if $p\left(\Pi_{i}(\omega)\right)>0$ for every $p \in \mathcal{P}$. We can observe that the concept of mutual absolute continuity is something thats depends on the given partitions, not on whether traders are myopic or more sophisticated (i.e. strategic).
} 
while $\omega_{1}$ can happen with strictly positive probability for some prior and $X\left(\omega_{1}\right)=1$ while in the other states it is zero, we have that the security is not constant.

In addition, compared to the definition of separable under $S E U$, as given in Ostrovsky (2012) and Chen et al. (2012), we can observe that under subjective expected utility assumptions due to linearity of the function $d_{\mathcal{P}}($.$) the definition is considerably simpler,$ without depending on the proper scoring rule or the order of the traders and the function $d_{\mathcal{P}}$ is simplified by an expectation. In addition, if the security is non-separable under $S E U$ then for $\mathcal{P}=\{p\}$ and any ordering of the traders, it is implied that it is nonseparable under ambiguity as well. That means, the notion of non-separability under ambiguity leaves out more securities, hence separable under ambiguity is stronger than separable under SEU.

The class of separable under ambiguity securities is not only contained in the class of separable under SEU securities (under the same information structure, given a proper scoring rule), but also the inclusion is strict. This is implied by the example provided above. $^{20}$ Finally, we can notice that the class of separable under ambiguity securities is non empty, because the constant security is always separable under ambiguity. In addition, an example of a non trivial security which is as simple as Arrow-Debreu whereas belonging to the class of separable under ambiguity securities is the security defined on the state space $\Omega=\left\{\omega_{1}, \omega_{2}, \omega_{3}\right\}$ as $X\left(\omega_{1}\right)=X\left(\omega_{2}\right)=1, X\left(\omega_{3}\right)=0$ under the partition structure $\Pi_{1}=\left\{\left\{\omega_{1}, \omega_{2}\right\},\left\{\omega_{3},\right\}\right\}, \Pi_{2}=\left\{\left\{\omega_{1}, \omega_{3}\right\},\left\{\omega_{2}\right\}\right\}$ and every continuous proper scoring rule.

\subsection{Main Result}

In this section, we state our main results. Our main result characterises the properties of the securities for which information is aggregated in a market populated by myopic traders.

Theorem 2.7. Consider the dynamic market described above and let security $X$, all traders be myopic with signal structure $\Pi$ and a strictly proper scoring rule s. Information is aggregated for every initial announcement $v_{0}, \mathcal{O}$ and $\mathcal{P} \in \mathcal{A}_{s, v_{0}, \mathcal{O}}$ if and only if $X$ is separable under ambiguity.

The proof of the Theorem 2.7 can be found in Appendix A.

In order to prove these two results a crucial lemma is needed. Lemma 2.8 adapts the results of Geanakoplos \& Polemarchakis (1982) and some results of Geanakoplos (1995) to the setting with ambiguity averse and myopic traders.

\footnotetext{
${ }^{20}$ Notice that when the set of priors is singleton then the ordering of traders can be ignored and the condition about the positive likelihood for every partition cell is satisfied trivially. Therefore, there is not confusion in comparing the two classes of securities.
} 
Lemma 2.8. Consider the dynamic market described above, a security $X$ and all traders be myopic with signal structure $\Pi$ who are ambiguity averse. In addition, let a strictly proper scoring rule $s$, initial announcement $y_{0}, \mathcal{O}$ ordering of traders and the common set of priors $\mathcal{P} \in \mathcal{A}_{s, y_{0}, \mathcal{O}}$. Then the following are true:

(i) For every state that can be realised, the public information $\mathcal{F}_{t}\left(\omega, y_{t-1}\right)$ will not be strictly refined from some $t^{\star}$ and onwards. Given $t^{\star}$, the corresponding round is $T^{\star}=$ $\min \left\{t \geq t^{\star}: t \bmod n=1\right\}$.

(ii) At each round, after the round $\left(T^{\star} \bmod n\right)+2$, the myopic predictions of the traders are constants, $v_{1} \in \mathbb{R}, \ldots, v_{n} \in \mathbb{R}$ respectively.

(iii) The traders reach an agreement, hence $v_{1}=\ldots=v_{n}$.

The proof of the Theorem 2.8 can be found in Appendix A.

This is essentially a generalisation of reaching a consensus of Geanakoplos \& Polemarchakis (1982), Cave (1983) and Bacharach (1985) and it is, in particular, related to the theorem about negation of asymmetric information in Geanakoplos (1995). It states that under any true state and in some finite steps the true state's information cell of the agents will reach its finest form. However, even though the traders can arrive at the common knowledge event, this does not imply that the traders, directly, agree on their predictions. They might even change their own prediction as well, depending on what is the previous prediction made. However, finally they will reach a consensus.

Intuitively, the difference between sequential announcements, among ambiguity averse traders, and the classic results in Geanakoplos \& Polemarchakis (1982), Geanakoplos (1995) and in Sebenius \& Geanakoplos (1983) is implied by the differences of their decision functions: in our setting decision functions are not just different across traders -this can be the case in Geanakoplos (1995), as well- but also they depend both on private information and in the announcement of the previous trader.

Having Lemma 2.8, the intuition of the proof for Theorem 2.7 can be thought of as consisting of two steps. Firstly, in finite rounds the information communication will stop and hence the agreement will be reached. Secondly, we will use that given the separability under ambiguity property of the security, this can happen only when the security is constant in all the remaining states. Finally, we conclude, using properties of the decision rule, that information gets aggregated.

\subsection{Concluding remarks}

This paper examines a dynamic market, based on MSR, with ambiguity averse traders, in terms of information aggregation. Using Gilboa \& Schmeidler (1989) MMEU the 
results indicate that trading particular classes of securities, among them are the ArrowDebreu securities, do not always aggregate information. Compared to similar results under subjective expected utility assumptions, this result is new and indicates that ambiguity creates issues in these markets.

The property of separable under ambiguity securities is easy to check, hence the results can be tested in the experimental lab in order to confirm whether information aggregation abnormalities are indeed the case under ambiguity aversion. Taking into account the wide range of applications of prediction markets, both the intuition of the paper and an experimental test of them would be of substantial interest for practical purposes.

Finally, the paper leaves open some interesting questions. Is this class of securities strategy proof? What happens when new traders starts trading entering the market? If the turn that traders predict in the market is random, does it affect how information gets aggregated? 



\section{Chapter 3}

\section{Information Aggregation with Strategic Traders}

This paper studies information aggregation in a dynamic trading model under ambiguity with finite, partially informed and ambiguity averse strategic traders. Instead of the commonly used assumption of common prior over the state space, here we assume that traders share a common set of multiple priors. We use as equilibrium the concept of revision proof equilibrium and we prove that under a converging assumption about the strategy profiles and restricting to pure equilibria, information gets aggregated in every such equilibrium when the securities are separable under ambiguity. Comparing to the class of securities that provides a similar characterisation with the standard subjective expected utility assumptions, namely the separable under SEU securities, the new class of securities turns out to be more restrictive and in particular, it is included in the class of separable under SEU ones. In fact, Arrow-Debreu securities do not belong to the class of separable under ambiguity securities, whereas they are separable under SEU. Hence it is concluded that information aggregation for strategic traders is not always possible under ambiguity aversion, even if information aggregation is the case under subjective expected utility assumptions.

\subsection{Introduction}

Information aggregation is a crucial property of financial markets. The extent to which it holds depends on several parameters, and of course on the strategic behaviour and rationality of the traders.

In the context of a fairly general class of dynamic markets, in particular the Market Scoring Rule (MSR), the answer is affirmative, Ostrovsky (2012). However, this is restricted by the the assumption of subjective expected utility and the assumption of a 
common and unique prior. However, ambiguity is inherent in these markets and hence a similar question was posed and answered, partially, in Chapter 2. In particular, it was restricted in the context of Gilboa \& Schmeidler (1989) model of ambiguity and of traders without strategic considerations.

In particular, the crucial question of examining whether the class of separable under ambiguity securities, as defined in Chapter 2, is strategy proof is still not answered. For separable securities under SEU that property holds, and in this chapter we are going to look towards deriving a similar result for the separable under ambiguity securities.

In Appendix B several examples are given illustrating that there are separable under SEU securities which when traded in a market, populated by myopic traders, information does not get aggregated. Using a particular equilibrium concept, we can see that the myopic behavior in those examples is, in fact, an equilibrium. In other words, we can conclude that when traders are strategic there exist separable under SEU securities that do not aggregate information. Among others, an example of a separable under SEU security with that property is the Arrow-Debreu security.

Furthermore, there is a lot of debate whether in prediction markets traders are myopic or strategic and therefore being the set of separable under ambiguity securities strategy proof has strong implication for the accuracy of these markets.

The first contribution of that chapter is to provide such a result. Intuitively, our result states that in Market Scoring Rule (MSR) markets with separable under ambiguity securities information gets aggregated, in pure converging strategies.

The compelling part of providing such a result in a setting under ambiguity is that in an extensive form game, which is the case for prediction markets, it is implied that time inconsistent decision should be taken, due the violation of dynamic consistency. This is because we assume Bayesian updating prior by prior. That makes considerably more complex to analyse a game. In particular, among others, two intuitive equilibrium concepts exist to model such a strategic interaction. The most widely used is the consistent planning approach, according to which each player optimises given both the other players' strategies and the future actions of her. An interpretation of such a concept is to consider the future actions of each player as different selves of the same player. The second one is the naive way which lets the decision maker to optimise the current payoff knowing that the actions might change in the next period. Finally, by providing an equilibrium concept for infinite incomplete information games under ambiguity is a second crucial contribution of our paper.

Consistent planning has been behaviorally characterised in Siniscalchi (2006) and is dominant in applications. However, it allows to optimise for one period deviations, leaving in that way a lot of deviation strategies without consideration. In particular, given a consistent planning equilibrium, it might be the case that there is an alternative 
strategy profile for a trader which, by changing her future actions, and not only current period's, can provide weakly better payoff to every future player's self, and strictly better payoff for at least one future self. In other words, there might be a profitable revision for the player, and her future selves, to make. In fact, we define an equilibrium concept in which these revision are taken into account. In the complete information setup this equilibrium was analysed in Ales \& Sleet (2014) and here we suggest a generalisation of the concept for settings with incomplete information.

A crucial issue with the incomplete information version of that equilibrium concept is how the beliefs should be updated especially at off equilibrium information sets. In particular, on the path of a revision proof equilibrium the beliefs are updated with Bayes rule prior by prior. In the same spirit, when a player finds herself in an information set off equilibrium path then we assume that beliefs are updated prior by prior whenever possible. Essentially, we follow Fudenberg \& Tirole (1991) in order to address this issue. In contrast to Ostrovsky (2012), for our result to hold, the off equilibrium beliefs are crucial in order to prove the result and this is because of the lack of properties of the myopic decision function under ambiguity.

Therefore, the main contributions of this paper are two. On the one hand, we suggest as equilibrium concept the revision proof equilibrium generalising it for incomplete information and infinite horizon games and restricting minimally the beliefs at off equilibrium information sets. We then apply this concept in order to prove the strategy proofness property of the separable under ambiguity class of securities, which is the main result of the paper.

The perspective that we take in this paper is to apply the equilibrium concept in the MSR game in order to study if information gets aggregated.

\subsubsection{Literature Review}

The literature of games under ambiguity can be thought as being divided in two parts. The first one consists of papers that are studying ambiguity about opponents' strategies or in other words about strategic ambiguity. The second one is games with ambiguity regarding the states of the world, namely environmental ambiguity. This paper belongs to the second class of papers.

The literature of dynamic games under ambiguity is fast growing as these structures are met in many economic applications. In particular, in Bose \& Renou (2011) Bose \& Daripa (2009) the effect of ambiguity aversion is studied in a mechanism design framework. The games in these papers are similar to the one it his paper but having finite time periods. The notion of equilibrium used is the consistent planning equilibrium with leaving unconstrained the off equilibrium beliefs, which is more similar to the 
Weak Perfect Bayesian Equilibrium and not Perfect Bayesian Equibrium. A similar equilibrium concept is used in Mele \& Sangiorgi (2015).

In Hanany et al. (n.d.) a more abstract approach is taken. The paper suggests two equilibrium concepts, namely Sequential Equilibrium with Ambiguity and Perfect Equilibrium with Ambiguity and proves some existence results. The existence refers to finite horizon. These equilibrium concepts are defined to have a component of sequential rationality (as in the standard subjective expected utility case) and a second component that involves the way that the beliefs are updated. The latter uses the results of Hanany \& Klibanoff (2009) in order dynamic consistency to hold and therefore the sequential rationality to be well defined. As a result of the updating rule, it is proved that the rationality component is equivalent to the one-shot-deviation principle, relating in that sense to the consistent planning equilibrium as well.

Furthermore, dynamic games are studied as well in Eichberger \& Kelsey (2004) and Dominiak \& Lee (n.d.) and their applications to signaling game. The models in these papers allow for both environmental and strategic ambiguity and one novelty of their paper is that the DempsterShafer updating rule, which they suggest, can be used to update the beliefs even after deviations.

Some additional papers that have analyzed incomplete information games with ambiguity sensitive preferences are Kellner \& Thordal-Le Quement (n.d.), Wolitzky (2014), Azrieli \& Teper (2011), Battigalli et al. (2015), Lopomo et al. (2009) and Sass 2013.

\subsubsection{Overview}

The paper is organised as follows. In Section 3.2 we are referring to the details of the trading model and the details about the introduction of ambiguity. In Section 3.2.1 we provide the details of the equilibrium concept, in Section 3.3 the notion of information aggregation and in Section 3.4 the properties of the new class of securities. Finally in

Section 3.5 we provide our main result. All proofs are contained in Appendix C and Appendix D.

\subsection{Model}

The model and the setting of this Chapter is the same with the one of Chapter 2. In particular, the trading environment is as in Section 2.1.3. The ambiguity and the myopic behavior are the same as defined in Section 2.1.4 and Section 2.1.5 respectively. 


\subsubsection{Equilibrium Concept}

This part is about defining formally the optimisation problems that strategic traders face. In particular, firstly, we give details about the decision function of myopic traders. Then, we define the equilibrium concept for the strategic traders.

\subsubsection{Equilibrium}

In this part of the paper the concept of equilibrium is discussed. Before we proceed with the details it is crucial to highlight here a considerable difference between Ostrovsky (2012) and our setting, regarding the information aggregation of strategic players. As it will be obvious in the proof of Theorem 3.6, when players are ambiguity averse then the off equilibrium behavior is important, as opposed to the setting under subjective expected utility assumptions. The reason for that is, on the one hand, the dependence of the decision function on the previous announcement, as it was highlighted in subsection ?? and, on the other hand, because dynamic consistency in Ostrovsky (2012) allows to check for deviation with all strategies in every continuation game (i.e. sequential rationality). Therefore on equilibrium path arguments like in Ostrovsky (2012) cannot be applied.

We begin first by defining the game and the notation that will be used latter. Next, we define the equilibrium concept that is used for the main result of the paper.

Consider a player $i$ who trades at periods $t_{i+n k}$, with $k \in \mathbb{N}$, given a strictly proper scoring rule $s$, MM's initial announcement $y_{0}$, an ordering $\mathcal{O}$ and a initial set of priors $\mathcal{P} \in \mathcal{A}_{s, y_{0}, \mathcal{O}}$. A pure strategy of the player is a sequence $y^{i}=\left(y_{i+n k}\right)_{k \in \mathbb{N}}$, where $y_{i+n k}$ : $\Pi_{i} \times Y^{i-1+n k} \longrightarrow Y$ is a measurable function, for every $k \in \mathbb{N}$. Therefore, strategies depend on the element of partition and on the history of predictions until time $t_{i-1+n k}$ (i.e. previous prediction).

We denote by $H^{i-1+n t}(\omega)$ the information set of player $i$ which is met when the realised state is $\omega$ and the history is $H^{i-1+n t}$. In particular:

$$
H^{i-1+n t}(\omega)=\left\{\left(H^{i-1+n t}, \omega^{\prime}\right): \omega^{\prime} \in \Pi_{i}(\omega)\right\}
$$

Therefore, the set of priors corresponding to that information set are essentially a set of priors over the $\Pi_{i}(\omega) .{ }^{21}$

For two histories $H^{n}=\left(y_{1}, \ldots, y_{n}\right)$ and $H^{m}=\left(y_{1}^{\prime}, \ldots, y_{m}^{\prime}\right)$ we denote $H \cup H^{\prime}=\left(y_{1}, \ldots, y_{n}, y_{1}^{\prime}, \ldots, y_{m}^{\prime}\right)=$ $H^{n+m}$, meaning the whole history consisted of the two components. In particular, the indices $n$ and $m$ are denoting the actions, $y_{1}, \ldots, y_{n}$ and $y_{1}^{\prime}, \ldots, y_{m}^{\prime}$ included in each history respectively. By $H^{i-1+n k} \cup H_{i-1+n t}^{i-1+n k}(y(\omega))$, for some $H^{i-1+n k} \in Y^{i-1+n k}$, we mean

\footnotetext{
${ }^{21}$ We will explicitly define the properties of the set of priors in Definition 3.3.
} 
the history, from $(i-1+n t)$-th until $(i-1+n k)$-th announcement (excluding the $(i-1+n t)$-th announcement), created by the profile strategy $y$ when the true state is $\omega$ and given $H^{i-1+n k}$. In addition, it is $H \cup H_{t}^{t}=H$ for every $t \in \mathbb{N}$ and history $H$. When the starting point for the history is the announcement of the market maker we just write $H^{i-1+n k}(y(\omega))$. Finally, denote by $\mathcal{H}=\left\{H \in Y^{m}: m \in \mathbb{N}\right\}$ the set of all histories. Hence $\mathcal{H}(\omega)$ the set of information sets when the realised state is $\omega$.

Furthermore, we denote

$$
\mathcal{F}_{H_{t_{0}}^{i-1+n k}(y(\omega))}=\left\{\omega^{\prime} \in \Pi_{i}(\omega): H_{t_{0}}^{i-1+n k}(y(\omega))=H_{t_{0}}^{i-1+n k}\left(y\left(\omega^{\prime}\right)\right)\right\}
$$

with $t_{0} \in \mathbb{N}, t_{0} \leq i-1+n k$ and given some history until $t_{0}$. This can be interpreted as the public information created, given the history until $t_{0}$, by following strategy's path from time $t_{0}$ until $t_{i-1+n k}$, when the realised state is $\omega$. Given a set of priors $\mathcal{P}$ then by $\mathcal{P}_{\mid E}$ is the set of prior by prior conditional priors conditional on the event $E$, assuming that every two priors in the set $\mathcal{P}$ are mutually absolute continuous with respect to $E$.

The equilibrium concept that is used is related to revision-proof equilibrium of Ales \& Sleet (2014). Due to time inconsistency of agents' preferences through out the time horizon (which applies to our setting because of the potential failure of dynamic consistency) a usual approach in the literature is to use the consistent planning approach. The idea behind the revision-proof equilibrium is to strengthen the concept of consistent planning in order to exclude some implausible equilibria. For example, it is plausible the consistent planning equilibrium to be such that there is no player, realised state, an alternative strategy for the player and a history such that every player's "self" after that history is weakly better payoff and strictly better at least one time. This is essentially defined as the revision-proof property.

However, in Ales \& Sleet (2014) revision proof equilibrium is referred to complete information setting, hence for our purposes we need to generalise this concept for incomplete information. Following the idea of Perfect Bayesian Equilibrium, the equilibrium will be an assessment $\left(y^{*}, \mathcal{P}\right)$, where the $y^{*}$ is the strategy profile and the $\mathcal{P}=\left(\mathcal{P}_{H(\omega)}\right)_{\omega \in \Omega, H(\omega) \in \mathcal{H}(\omega)}$ a system of convex and compact set of priors for each information set. In short, instead of the idea of "sequential rationality" here we have the revision proof concept, with the expectation of the continuation games being based on the MMEU. In addition to that, we will assume that the beliefs are updated prior by prior whenever possible. In particular the definition of the equilibrium concept is given below in Definition 3.3.

Firstly, we will give the definition of consistent planning equilibirum, that is often met in the literature, and elaborating on some issues regarding that concept in our setting, we then define our main equilibrium concept. In the formulation of the following definition we are based on the the definition of sub game perfection of Ales \& Sleet (2014), generalising it for incomplete information. In the formulation of our notion of consistent 
planning we wanted to include the idea of one shot deviation. In Bose \& Renou (2011) and Hanany et al. (n.d.), among others, consistent planning is defined based on that one shot deviation, hence making these definitions, from that point of view, considerably similar to ours. However, differences on how beliefs are updated exist with Hanany et al. (n.d.), and in terms of the infinite horizon with both of them.

Definition 3.1. If the state chosen by nature is $\omega$, the strategy of players is $y=$ $\left(y^{1}, \ldots, y^{n}\right)$ and the history is $H^{i-1+n t}$ then the continuation payoff of player $i$ at round $t$, given profile $y^{-i}$, is given by

$$
\begin{aligned}
& V_{i+n t}\left(H^{i-1+n t},\left(y^{i}, y^{-i}\right), \omega\right)=\min _{p \in \mathcal{P}_{H^{i-1+n t}(\omega)}} E_{p}\left[\sum _ { k = t } ^ { \infty } \beta ^ { n k - n t } \left(s \left(y _ { i + n k } \left(\Pi_{i}(\omega), H^{i-1+n t} \cup\right.\right.\right.\right. \\
& \left.\left.\left.\left.H_{i-1+n t}^{i-1+n k}\left(y\left(\omega^{\prime}\right)\right)\right), X\right)-s\left(y_{i-1+n k}\left(\Pi_{i-1}\left(\omega^{\prime}\right), H^{i-2+n t} \cup H_{i-2+n t}^{i-2+n k}\left(y\left(\omega^{\prime}\right)\right)\right), X\right)\right)\right] .{ }^{22}
\end{aligned}
$$

Definition 3.2. For $0<\beta<1$ we define the assessment $\left(y^{*}, \mathcal{P}\right), y^{*}=\left(y^{*^{1}}, \ldots, y^{*^{n}}\right)$ to be a pure Consistent Planning equilibrium:

If there is no $i, \omega$, such that there exists $p \in \mathcal{P}$ with $p(\omega)>0, t \in \mathbb{N}$, history of predictions $H^{i-1+n t} \in Y^{i-1+n t}$ and alternative action $a \in Y$ such that:

(i) Given the $-i$ equilibrium strategies it is $V_{i+n t}\left(H^{i-1+n t} \cup\{a\},\left(y^{*^{i}}, y^{*^{-i}}\right), \omega\right)>$ $V_{i+n t}\left(H^{i-1+n t},\left(y^{*^{i}}, y^{*^{-i}}\right), \omega\right)$.

and

(ii) Let state $\omega, i \in\{1, \ldots, n\}, k \in \mathbb{N}$ and history $H^{i-1+n k}$. It holds that for every $\omega^{\prime} \in$ Supp $\mathcal{P}_{H^{i-1+n k}(\omega)}$ every two priors in $\mathcal{P}_{H^{i-1+n k}(\omega)}$ are mutually absolute continuous with

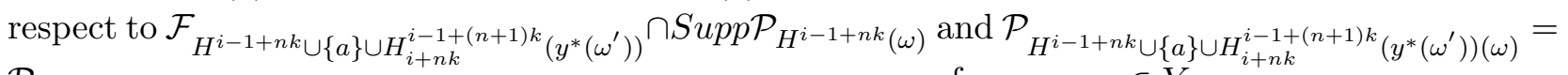
$\mathcal{P}_{H^{i-1+(n+1) k}(\omega)} \mathcal{F}_{H^{i-1+n k} \cup\{a\} \cup H_{i+n k}^{i-1+(n+1) k}\left(y^{*}\left(\omega^{\prime}\right)\right)} \cap \operatorname{Supp}_{H^{i-1+n k}(\omega)}$ for every $a \in Y$.

Condition ( $i$ i) of Definition 3.2 states that prior by prior updating should be used whenever possible. In particular, it states that prior by prior updating is used on equilibrium path. This is because if the $a$ is the respective equilibrium action of $i$ then the mutual absolute continuity with respect to $\mathcal{F}_{H^{i-1+n k} \cup\{a\} \cup H_{i+n k}^{i-1+(n+1) k}\left(y^{*}\left(\omega^{\prime}\right)\right)} \cap$ Supp $\mathcal{P}_{H^{i-1+n k}(\omega)}^{23}$ means that prior by prior updating is well defined. Then by requiring

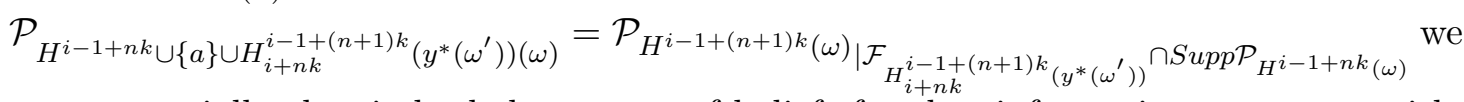
state essentially that indeed the system of beliefs for that information set agrees with the prior by prior updating.

On the other hand, (ii) of Definition 3.2 clarifies the issue of the "whenever possible", met in the literature of Perfect Bayesian Equilibrium, Bonanno (2013) among others. In particular, when $a$ is not necessarily the equilibrium action of $i$, the $(i i)$ of Definition 3.2 clarifies when we assume the beliefs to be updated prior by prior. Indeed, in our case the

\footnotetext{
${ }^{22}$ We are referring to continuation payoff at time $i+n t$, by meaning the continuation payoff divided by $\beta^{i+n t}$.

${ }^{23}$ The definition of support is given in Section 2.1.4.
} 
game is sequential, hence if player $i$ deviates at time $i+n t$, then player $i$ knows that she deviated. Therefore, given that $-i$ follow their equilibrium strategies, (ii) of Definition 3.2 urges player $i$ to update like a Bayesian prior by prior at her next information set.

Regarding $(i)$ of Definition 3.2 we can observe that the optimisation of each player considers only deviations from the equilibrium action of the current round (that is the alternative $a \in Y$ in the Definition 3.2).

However, even under subjective expected utility assumptions, in Ostrovsky (2012), one of the most crucial arguments for proving the information aggregation result in the MSR model lies in the property of sequential rationality, meaning that players can optimise across any alternative action at any round. Given that, it is implied that players are optimising against the alternative strategy of playing whatever the previous prediction was. This is a deviation that in our equilibrium concept we would like to take into account, as it is a crucial property of the MSR model. In particular, it can be interpreted as an opt-out strategy.

In Definition 3.3 we define our notion of equilibrium. It has a common part with Definition 3.2, which is part (iii) and (ii), respectively. However, $(i)$ and (ii) of Definition 3.3 are replacing the weaker $(i)$ of Definition 3.2.

In particular, revision proofness allows for checking across all possible deviations. Intuitively, revision proofness states that even if a future self arbitarily close to infinity does not find a deviation optimal, then that deviation is blocked even in the case that previous players were better off under the deviation. On the other hand, according to consistent planning equilibrium each self is isolated and she plays a game against all others, hence from that point of view it is plausible to assume that only one shot deviations should be considered.

In short, the essential difference between these two concepts is that in a revision proof equilibrium players are willing to coordinate their actions in case everyone is better off by that, whereas in consistent planning equilibrium coordination is not considered.

Definition 3.3. For $0<\beta<1$ we define the assessment $\left(y^{*}, \mathcal{P}\right), y^{*}=\left(y^{*^{1}}, \ldots, y^{*^{n}}\right)$, to be a pure Revision-Proof equilibrium if there is no $i, \omega$, such that there exists $p \in \mathcal{P}$ with $p(\omega)>0$, history of predictions $H^{i-1+n t} \in Y^{i-1+n t}$ for some $t \in \mathbb{N}$, and alternative strategy $y^{i}=\left(y_{i+n k}\right)_{k \in \mathbb{N}}$ such that:

(i) For all $r \in \mathbb{N}, H^{n r} \in Y^{r n}$ and given that $-i$ play their equilibrium strategies it is $V_{i+n t+n r}\left(H^{i-1+n t+n r},\left(y^{i}, y^{*^{-i}}\right), \omega\right) \geq V_{i+n t+n r}\left(H^{i-1+n t+n r},\left(y^{*^{i}}, y^{*^{-i}}\right), \omega\right)$

(ii) The inequality is strict for at least one history $H^{n r} \in Y^{n r}$ and

(iii) Let a state $\omega, i \in\{1, \ldots, n\}, k \in \mathbb{N}$ and history $H^{i-1+n k}$. It holds that for every $\omega^{\prime} \in$ Supp $\mathcal{P}_{H^{i-1+n k}(\omega)}$ every two priors in $\mathcal{P}_{H^{i-1+n k}(\omega)}$ are mutually absolute continuous with 
respect to $\mathcal{F}_{H^{i-1+n k} \cup\{a\} \cup H_{i+n k}^{i-1+(n+1) k}\left(y^{*}\left(\omega^{\prime}\right)\right)}^{\cap S u p p} \mathcal{P}_{H^{i-1+n k}(\omega)}$ and $\mathcal{P}_{H^{i-1+n k} \cup\{a\} \cup H_{i+n k}^{i-1+(n+1) k}\left(y^{*}\left(\omega^{\prime}\right)\right)(\omega)}=$

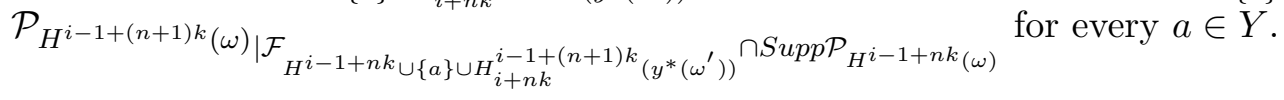

We can observe that if a strategy profile is not consistent planning equilibrium then there exists player $i, \omega^{*}$ that can be realised as true state, a round $t$ and history $H^{i-1+n t}$ such that for some deviation $a$ the equality of Definition 3.2 in (i) holds. Define a deviation strategy for player $i$ which is the same as her equilibrium for every $\omega$ that can be realized and any history, except for the history $H^{i-1+n t}$ when $\omega^{*}$ is realized, in which case it prescribes playing $a$. Under this new strategy everyone is getting the same payoff under any history and true state, but for $\omega^{*}$ and history $H^{i-1+n t}$ the player $i$ get a strictly better payoff. Therefore, the strategy is not revision proof.

For the results about strategic traders we restrict to pure revision proof equilibrium that satisfies a certain assumption. Intuitively, the assumption states that the on equilibrium path strategic predictions of the traders should converge to a real number. However, we do not assume that the real number is the same for every trader. We name such an equilibrium as converging strategies equilibrium.

It is out of the scope of this paper to study the equilibrium existence in MSR games with ambiguity. Therefore, we can think of two classes of potential equilibria: the first one is when the strategic prediction of the traders are converging, on equilibrium path, to a real number (the limit is, potentially, different among traders) and the second one is when the predictions are not converging for at least one trader and state. In this paper we want to restrict attention to the first class, as in the second class of equilibria, obviously, information cannot get aggregated.

In Ostrovsky (2012), there was no separation between those two classes. In that way, the information aggregation result for separable under SEU securities that was proven, essentially implies a necessary condition for the existence of equilibrium in MSR games under subjective expected utility. In fact, the necessary condition is that those equilibria not only belong to the fist class but also the real number that strategic predictions are converging are the same for all traders (and essentially the true value of the security).

In our case, we will restrict to the first class of equilibria. The reason for making such an assumption is the lack of properties that hold under subjective expected utility. In particular, the arguments about instant opportunity and arbitrage in Ostrovsky (2012) use heavily properties inherent to subjective expected utility and that can bypass the issue whether in he equilibrium the predictions for each trader converge somewhere. ${ }^{24}$

The assumption in our case takes the following form.

\footnotetext{
${ }^{24}$ Although the question is out of the scope of the thesis, my conjecture of existence of equilibrium it is not quite strict. The particular structure of the model (for example strictly proper scoring rule) provides potentially strong properties to use in a relevant proof.
} 
Assumption (converging strategies):

Let $y^{*}=\left(y^{*^{1}}, \ldots, y^{*^{n}}\right)$ be an equilibrium strategy profile for the players. For every $i=1, \ldots, n$ and every $\omega \in \Omega$, for which there exists $p \in \mathcal{P}$ with $p(\omega)>0$, there exists measurable function $y_{i, \infty}^{*}: \Pi_{i} \times Y^{\mathbb{N}} \longrightarrow Y$ such that $\lim _{k} y_{i+n k}^{*}\left(\Pi_{i}(\omega), H^{i-1+n k}\left(y^{*}(\omega)\right)\right)=$ $y_{i, \infty}^{*}\left(\Pi_{i}(\omega), H^{\infty}\left(y^{*}(\omega)\right)\right)$.

\subsection{Information Aggregation}

In this part of the paper we formally define the concept of information aggregation. In addition we compare it with the respective definition given in Ostrovsky (2012) and Chen et al. (2012).

Definition 3.4. Information gets aggregated with respect to security $X$, information partition $\Pi$, strictly proper scoring rule $s$, initial announcement $y_{0}$, order of traders $\mathcal{O}, \mathcal{P} \in \mathcal{A}_{s, y_{0}, \mathcal{O}}$ and pure equilibrium profile of strategies if the sequence of equilibrium predictions $y_{1}, \ldots, y_{k}, \ldots$ converges to the true value of the security, and the set of states that this does not hold is of zero probability for all priors in $\mathcal{P}$.

The definitions that were provided in Chen et al. (2012) and Ostrovsky (2012) were similarly stated. However, their definition tries to accommodate mixed strategies, which is not the case with our definition. In addition, in our setting we have a compact and convex set of priors that, in general, is not singleton. That is the reason why we should impose the regularity property as well.

\subsection{Separability Under Ambiguity}

The topic of this section is to give the properties needed to be satisfied by the securities in order to be able to characterise information aggregation for every set of priors. The condition is motivated by the example below.

The notion of separability under SEU, as given under subjective expected utility assumptions, Chen et al. (2012) and Ostrovsky (2012), has already been given in Definition 2.5.

However, this concept of separable under SEU securities is not enough to guarantee information aggregation for every set of priors for ambiguity averse traders, when they are myopic, as illustrated in the Example of section 2.3.

In particular, this example illustrates that an Arrow-Debreu security might fail to aggregate information for ambiguity averse myopic traders. In addition, we can observe that the myopic behavior in the previous example is a revision proof equilibium, hence 
concluding that in a market with an Arrow-Debreu security information does not get aggregated even if traders are strategic. ${ }^{25}$

In fact, the new notion of separability that we suggest here in order to achieve information aggregation for strategic traders is the same as Definition 2.6, and for completeness purposes it is defined again below.

Definition 3.5. A security $X$ is called non-separable under ambiguity under partition structure $\Pi$ and proper scoring rule $s$ if there exists an ordering of traders $\mathcal{O}$, a set $\mathcal{P} \subseteq \Delta(\Omega)$, which is convex compact and mutually absolute continuous with respect to $\Pi_{i}$ for every $i=1, \ldots, n$, and $v$ such that:

(i) $X$ is not constant at $\{\omega \in \Omega$ : there exists $p \in \mathcal{P}$ with $p(\omega)>0\}$

(ii) For every $i=1, \ldots, n$ it is $d_{\mathcal{P}}\left(\Pi_{i}(\omega), v\right)=v$ for every $\omega$, such that there exists $p \in \mathcal{P}$ with $p(\omega)>0$.

\subsection{Main Result}

In this section, we state our main results. The first result completely characterises the properties of the securities that can aggregate the information in a market populated by myopic or strategic traders.

Theorem 3.6. Consider the dynamic market as described above and let security $X$, partition structure $\Pi$, proper scoring rule $s$ and discount factor $\beta \in(0,1)$ :

(i) If security $X$ is separable under ambiguity, then for every initial announcement $v_{0}, \mathcal{O}$, $\mathcal{P} \in \mathcal{A}_{s, v_{0}, \mathcal{O}}$ and in any pure converging strategies revision proof equilibrium information gets aggregated.

(ii) If security $X$ is not separable under ambiguity, then there exists announcement $v_{0}$, $\mathcal{O}, \mathcal{P} \in \mathcal{A}_{s, v_{0}, \mathcal{O}}$ and a pure converging strategies revision proof equilibrium such that information does not get aggregated.

The proof of Theorem 3.6 is in Appendix C.

In order to prove these two results a crucial lemma is needed. Lemma 2.8 adapts the results of Geanakoplos \& Polemarchakis (1982) and some results of Geanakoplos (1995) to the setting with ambiguity averse and myopic traders. Although it is referred to myopic traders, in the proof of Theorem 3.6, we use the same arguments in order to have a result similar to $(i)$ for strategic players.

\footnotetext{
${ }^{25}$ The arguments to conclude that it is indeed a revision proof equilibrium are the same as given in the proof of Theorem $3.6(i i)$, in Appendix C, for $v=0$.
} 
The proof of Theorem 3.6 is involved and proceeds in three steps. Firstly, we will conclude that in a revision proof equilibrium the continuation games for each player $i$, at every realised true state and after any history that the player trades (which is interpreted as a separate self of $i$ ) should be a non negative real number. Then, using the fact that the strategies converge and properties of the MSR we conclude that the continuation payoffs should converge to zero. Finally, we conclude that in the limit the revision proof predictions are the myopic ones and then by separability under ambiguity we can show that information gets aggregated.

\subsubsection{Myopic Behavior can be Revision-Proof}

In this part of the paper two results are given, which relate the myopic behavior with a pure revision equilibrium, when a separable under ambiguity security is traded. In particular, we will prove that when, for every state that can be realised, the finest public information -while player are on equilibrium path- is achieved at the end of the first round, then on equilibrium path players are myopic, when the discounting factor $\beta \in\left(0,\left(\frac{1}{3}\right)^{1 / n}\right)$, where $n$ is the number of players. The second result is a generalisation of the previous and states that after achieving the finest public information set -while players are on equilibrium path- they are myopic, when $\beta \in\left(0,\left(\frac{1}{3}\right)^{1 / n}\right)$.

Theorem 3.7. Let $X$ be a separable under ambiguity security. Consider a pure strategy revision proof equilibrium, satisfying converging strategies assumption, and $\beta \in$ $\left(0,\left(\frac{1}{3}\right)^{1 / n}\right)$. Then players are myopic on equilibrium path, from the time the finest information is reached (for all) and onwards.

Theorem 3.8. Let $X$ be a separable under ambiguity security. Consider a pure strategy revision proof equilibrium, satisfying converging strategies assumption, $\beta \in\left(0,\left(\frac{1}{3}\right)^{1 / n}\right)$ and assume for every state that can be realised, public information reaches its finest refinement in the first round. Then players are myopic on equilibrium path.

The proofs of the theorems above are contained in Appendix D.

\subsection{Concluding remarks}

The main scope of this paper is to study a dynamic market, based on MSR, with ambiguity averse and partially informed traders in terms of information aggregation. Using Gilboa \& Schmeidler (1989) model, the results indicate that when either myopic or strategic traders are participating in the market, information does not always get aggregated, unless the security of the market is separable under ambiguity. Compared to similar results under subjective expected utility assumptions, this result is new and 
indicates that ambiguity aversion creates an extra layer that precludes information aggregation.

Finally, the paper leaves open some interesting questions. For example, can the assumption of converging strategies be avoided, hence more technical arguments should be used in the proof for strategic traders? What happens, in terms of information aggregation, when the players do not play in fixed turn but randomly? Finally, does an MSR game have an equilibrium? 



\section{Chapter 4}

\section{Experiment}

This paper studies information aggregation in a dynamic market under ambiguity. Studying the effect of ambiguity on information aggregation when a "separable under SEU" security, in particular an Arrow-Debreu security, is traded in the market, we find evidence that its price is significantly less accurate in case the fundamentals of the security are ambiguous. On the contrary, when introducing in the market a "separable under ambiguity" security, in fact a modification of the Arrow-Debreu security making insider one of the two traders, we find evidence that ambiguity about the fundamentals does not affect market's degree of information aggregation. These results confirm theoretical predictions given in Chapter 2 and Chapter 3.

\subsection{Introduction}

A key question regarding the design of financial markets is whether they have the ability to aggregate and reveal dispersed information. In particular, if information is dispersed among all traders, will their trading behavior lead the market to aggregate information, pricing the securities as if their information was pooled?

There are financial markets being created for taking advantage of the information aggregation property of securities prices. These markets are usually called prediction markets. In these markets bets are sold and bought hence moving the prices to their true value. Hence, the price can be interpreted as a forecast for a particular future event.

The particular form of the bets depend on the market model implemented. In fact, among others, two well knows market formats are more usual. Firstly, the continuous price auction which are commonly met in practice, but although it is a familiar market format, it is complex for the analyst and the traders to understand its dynamics and analyse it. Secondly, the Market Scoring Rule (MSR) is implemented more and more as an alternative market model used in practice. Its advantage over the continuous 
price auction is that it does not depend crucially on market liquidity (the market maker admits to have bounded losses) and are more tractable in terms of analysis.

The particular market implementation of MSR can vary. On the one hand, it is the probability based market Hanson (2003), Dimitrov \& Sami (2008) and Chen et al. (2010) among others. On the other hand is the security based market Ostrovsky (2012), Chen et al. (2012), which is the model of Chapter 2 and Chapter 3. The difference between those markets is that in the latter predictions about the value of a specific security are announced, while in the former the beliefs of each trader. In this Chapter, we use the control environment of the experimental laboratory to draw conclusion with regards to the security based model.

Restricted to that specific class of dynamic markets and to subjective expected utility assumptions for the fundamentals of the security, Ostrovsky (2012) and Chen et al. (2012) defined the class of separable under SEU securities. It was proved that when such securities are traded in the market, information gets aggregated. By relaxing the assumption of subjective expected utility and allowing for ambiguity aversion, the class of separable under ambiguity securities was defined in Chapter 2 and Chapter 3. It was proved that when such securities are traded in the market then information gets aggregated. It was proved, as well, that it is not, in general, true, in a setting with ambiguity, that with separable under SEU securities information gets aggregated.

In fact, Arrow-Debreu securities, which are separable under $S E U$, do not always aggregate information in a setting with ambiguity, as opposed to the case of subjective expected utility. However, a modification of an Arrow-Debreu security can aggregate information either with or without the presence of ambiguity. In particular, the experiment will be based on a model of three states of the world $\Omega=\left\{\omega_{1}, \omega_{2}, \omega_{3}\right\}$. The modification of the Arrow-Debreu security that we use is a security of the type $Y=(1,1,0)$. We consider it as a modification of the Arrow-Debreu security $X=(1,0,0)$ because it gives an extra payoff of 1 for the state $\omega_{2}$.

The main scope of this Chapter is to experimentally test these theoretical predictions. In other words, the ultimate scope is to test the hypothesis that ambiguity affects the degree of information aggregation for a separable under SEU security but not for a separable under ambiguity security.

\subsection{Literature Review}

A large number of experiments have been conducted to measure the efficiency of information aggregation in financial markets and prediction markets. Most of them have studied either double-auctions or parimutuel markets. 
Firstly, Plott \& Sunder (1988) studies and experimental double-auction, in order to test the theoretical predictions of REE. The results documented in the paper are mixed. In some treatments the markets do not reach REE. However, other treatments reach the equilibrium.

In Forsythe \& Lundholm (1990) an explanation of Plott \& Sunder (1988) mixed results was given.. The main result of that paper was to give sufficient conditions which are needed to be satisfied in order REE market's model to predict correctly the equilibrium. The results indicated that common knowledge of payoffs along with experience are giving a positive result.

In Plott et al. (2003) the question of information aggregation is set in the context of a parimutuel market. The results indicate that the more we are moving to more complex environments, the less information gets aggregated. Analysing the weak degree of information aggregation it is concluded that traders are bluffing and they participate in a waiting trading behavior, which both affect negatively the aggregation of information.

Our experimental design is also related to Hanson et al. (2006). This paper documents findings regarding manipulation in prediction markets. The information structure given in that paper is similar to ours. In fact, the private information is given by letting traders know, at each round, which state is not realised.

However our work differs in a main dimension from all these studies. They are using either double auctions or parimutuel markets. On the other hand we use the MSR.

In McKelvey \& Page (1990) the theoretical prediction of McKelvey \& Page (1986b) are tested in a market similar to MSR. In fact, the participants are incentivised by using a proper scoring rule,. This is a communication process very similar to Chen et al. (2012) and Ostrovsky (2012). The results suggest that there is a clear evidence that the information is not pooled in the marker, on the contrary of what theory predicts. However, there is also clear evidence that the public information that is revealed in the market augment each private information.

Finally, another closely related paper is Jian \& Sami (2012). It provides results regarding information aggregation of the MSR of Hanson (2003), under various hypotheses. For example, it is tested whether the trading order, the trading mechanism and the signal distributions affect the efficiency of information aggregation. They test as well if there exist evidence for results like Chen et al. (2012) and Dimitrov \& Sami (2008). In particular, it is tested whether the signal structure is related to myopic or bluffing trading. 


\subsection{Experimental Design}

For our experiment we recruited 124 subjects who were all students at the University of Southampton. Each student participated only in one treatment. Instructions were read publicly before the experiment. The instructions included details about the payoffs, the information structure according to the treatment and the software's computer interface. After the instructions, a paper based quiz was delivered to all subjects and the experimenter checked each subject's answers in person. Finally, trial rounds were given so that subjects can be fully familiar with the software before the main experiment starts. Communication among the subjects was strictly forbidden throughout the experiment. The experiment was programmed and conducted with the software z-Tree Fischbacher $(2007) .^{26}$

Our design and approach is similar to Jian \& Sami (2012), although the hypotheses are different. Our hypotheses are more similar to McKelvey \& Page (1990). Our experiment consists of 4 treatments, shown in the table below. In each session, which consisted of 5 trials and 20 rounds, 16 subjects participated. They were divided into 2 groups, which remained the same throughout the session. By doing so we got two independent observations at each session. For each treatment two sessions were ran.

\begin{tabular}{ccc}
\multicolumn{3}{c}{ Treatments } \\
\hline \multirow{3}{*}{ Security $X$} & Security $Y$ \\
\cline { 2 - 3 } Expected Utility & $E U-X$ & $E U-Y$ \\
\cline { 2 - 3 } Ambiguity & $A m b-X$ & $A m b-Y$ \\
\hline
\end{tabular}

FiguRE 4.1: Treatments.

At the beginning of the experiments subjects were informed that they were endowed with 40 units of experiment currency and that the exchange rate was 100 units of experimental currency to 1 GBP.

At the beginning of each treatment, the sixteen subjects were randomly grouped into two groups, with eight subjects each. At the beginning of each round the eight subjects were randomly paired, without been told with whom they were matched or whether they had been paired before. In each pair, one subject was randomly assigned as trader 1 and the other as trader 2. ${ }^{27}$

At the beginning of each round a ball was drawn from a box, which included 90 balls in total. ${ }^{28}$ The colours of the balls contained in the box are red, green or yellow. For

\footnotetext{
${ }^{26}$ The instructions can be found in Appendix E.

${ }^{27}$ In the third session we used 12 instead of 16 subjects. The subjects were divided into two pairs of 6 subjects each.

${ }^{28}$ Following Jian \& Sami (2012) the drawn ball was selected before the experiment, in order to control for differences in learning effects across treatments. We used the random.org pseudo-random number generator and we tested, against several other seeds, the number sequence that was used using the Kolmogorov-Smirnov.
} 
the treatment of ambiguity less information was disclosed to the traders regarding the composition of the box, as opposed to the expected utility treatment. We will refer to the box in the treatment of ambiguity as ambiguous box and to the box in the treatment of expected utility as risky box, separating in that way the difference in the information disclosed.

Two securities have been used, security $X$ and $Y$. Security $X$ takes the following values: $X($ red $)=100, X($ green $)=0$ and $X($ yellow $)=0$. On the other hand, security $Y$ takes the following ones: $Y($ red $)=100, Y($ green $)=100$ and $Y($ yellow $)=0$. Security $X$ belongs to the class of separable under $S E U$ securities (for any strictly proper scoring rule, hence for the quadratic we use in the experiment), defined in Ostrovsky (2012), while security $Y$ belongs to the class of separable under ambiguity securities (given the quadratic strictly proper scoring rule use in he experiment), defined in Chapter 2 and Chapter 3, but it is not separable under SEU.

The market model followed the Market Scoring Rule (MSR) as illustrated in Ostrovsky (2012) and Chapter 2 and Chapter 3. The traders in each pair were called to, sequentially, submit their prediction (a number between 0 and 100) regarding the true value of the security, according to the ball drawn, for several times. Depending on whether the subject was trader 1 or trader 2 some private information was given to him/her, as shown in Figure 2. Similar information can be found in Hanson et al. (2006), among others. We can observe that by pooling together the private information of trader 1 and of trader 2 , we know the colour of the drawn ball.

\begin{tabular}{c|c|c}
\multicolumn{3}{c}{ Information Structure } \\
\hline Ball Drawn & Signal for Tader 1 & \multicolumn{1}{c}{ Signal for Tader 2 } \\
\hline Red & Not Yellow & Not Green \\
Green & Not Yellow & Green \\
Yellow & Yellow & Not Green \\
\hline
\end{tabular}

FiguRE 4.2: Information structure.

At each round, the subjects were predicting at least 3 times each. That guaranteed that we would have enough observations for our purposes. However, in order to accommodate the infinite horizon model used in Ostrovsky (2012) and Chapter 2 and Chapter 3 we followed the design of Fréchette \& Yuksel (2013), Cabral et al. (2014) and Vespa (2011). In particular, for the first 6 periods each trader had an explicit discounting in trader's payoff. For the rest of the periods the payoffs were not discounted explicitly, but the participants were informed about the probability of moving to these stages. The Figure 3 below illustrates the details.

\subsubsection{Risk Vs Ambiguous Box with Security X}

For the treatment with the risky box, the proportions of the balls included in it were given. In fact, it was disclosed that 30 red, 30 green and 30 yellow balls were included 


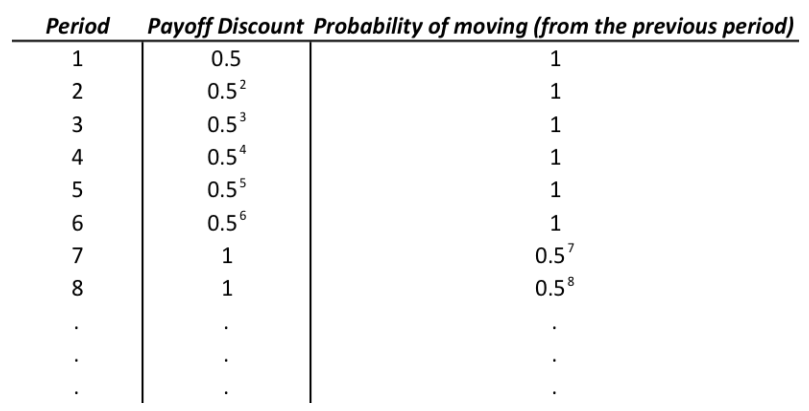

Figure 4.3: Discounting.

in the box. On the other hand, for the ambiguous box the exact proportions were unknown. On the contrary, the participants were informed that there were between 0 and 30 red balls, between 20 and 70 green balls and between 20 and 70 yellow balls when the security $X$ was used, and between 1 and 30 red balls, between 19 and 70 green balls and between 19 and 70 yellow balls when the security $Y$ was used. ${ }^{29}$

As it was proved in Chapter 2 and Chapter 3 when the market, with the ambiguous box, starts with the announcement 0 (which is always the case in our experiment) and traders are ambiguity averse, as in Gilboa \& Schmeidler (1989), market cannot aggregate their private information in case the drawn ball is red. Whenever the ball is either green or yellow, though, information gets aggregated.

However, with the risky box it is known, for example by Ostrovsky (2012) and many others, that market aggregates the private information of each trader, therefore information aggregation does take place for any colour of the drawn ball.

Therefore based on the theoretical results we expected that the predictions of the treatments with the ambiguous box would be significantly less accurate compared to the predictions of the treatments with the risky box in case the ball is red, but similar in case the ball is either green or yellow.

\subsubsection{Risk Vs Ambiguous Box with Security Y}

The underlying idea of these two treatments is the same as the one explained in Section 4.3.1, but using a different security $Y$. The only difference is that in the treatment with security $Y$ and ambiguity different information was disclosed. In particular, in order to be consistent with the theoretical model, which assumes prior by prior updating, the participants were told that there are between 1 and 30 red balls, between 19 and 70 green balls and between 19 and 70 yellow balls.

According to Chapter 2 and Chapter 3, there should not be significant evidence on the difference between the market with the risky and the ambiguous box. In fact, irrespective

\footnotetext{
${ }^{29}$ The difference between the ambiguous boxes is explained in Section 4.3.2.
} 
of the drawn ball's colour, information, in theory, gets aggregated. Hence we expected that the predictions would be the same either being predictions from the sessions with the risky treatment or not.

\subsection{Analysis Metrics and Hypotheses}

We define the true value of the security, depending on the realised colour of the ball, as the best prediction a trader could submit. We can easily observe that given the information structure used in our experiment, by pooling their private information traders can find out the colour of he drawn ball. In theory such a MSR market would converge towards the true value under some conditions, Chapter 2, Chapter 3 and Ostrovsky (2012). Given that, we will use the true value of the security as a benchmark for measuring how accurate predictions are. In particular, the accuracy of a prediction is measured as the Absolute Distance (AD) of the prediction from the true value of the security. Similar metric, the mean squared error, was used in Jian \& Sami (2012).

Having as market benchmark the market with risk, we analyse the market with ambiguity using the metric of the $\mathrm{AD}$. If the $\mathrm{AD}$ in market with ambiguity is larger than the corresponding of the risky market, then we conclude that the degree of information aggregation is better in the risky market.

Having defined the metric of the analysis we can now define the hypotheses of our experiment formally. The main idea is to test whether ambiguity has an effect on the accuracy of the predictions. In addition, we test whether the design of separable under ambiguity securities is robust enough in order to overcome that effect.

HYPOTHESIS 1. When the drawn all is Red then the market with ambiguity and security $X$ does not aggregate information as effectively as the market with risk. However, the degree of information aggregation is the same when the drawn ball is either Green or Yellow.

HYPOTHESIS 2. When the security is $Y$ then the two markets aggregate information at the same degree, for any colour of the drawn ball.

In the following section, we report the results of our experiment. Our experimental design implies four independent observations for each treatment. Hence we use permutation test, which is a type of nonparametric test, in order to conduct the comparisons of markets' performances. Permutation tests do not make any assumption regarding the independent data points and it can be easily verified that having four data points for each of our treatment, the lowest p-value possible is 0.014 . 


\subsection{Experimental Results}

In the following subsections we report and discuss the results of the experiment. We begin with an overview of the performance of the effects on the treatments and then we proceed with the results of the statistical tests with regards to the hypotheses.

\subsubsection{Results without Learning Effects}

Our experimental design implies that each round the same colour of the ball is drawn across sessions. Hence, two approaches are employed in order to analyse the data. Firstly, for each colour we are focusing on the final rounds in order to analyse the results, hence avoid incorporating the noise while the subjects are learning the game and the software. Secondly, we explicitly take into account learning effects, and undertake the statistical analysis accordingly.

In particular, for the red balls we are focusing on the last four rounds, for the green to the last 3 and for the yellow balls on the last 3 rounds. In addition, we create a column with the average $\mathrm{AD}$, per session, over the number of rounds we want to focus at each time. Finally, we combine all these average columns into one, by taking their average. We refer to these data as the average data. The data structure, as explained, can be found in Appendix E.

In Figure 4.4 the box plots for the two treatments are shown, when the security is $X$. We can observe that when the drawn ball is red then the average, the last two rounds, the last three rounds and mostly the final round in the ambiguity treatment with security $X$ are yielding higher absolute distances, as compared to the expected utility treatment with security $X$. In relation to our first hypothesis, that would mean that when the ball is red the market with ambiguity is performing significantly worse that the market with risk.

On the other hand, the rest markets do not seem to differentiate significantly with regards to the existence of ambiguity or not. In fact, we can see more mixed effects and hence suggesting the for green and yellow balls the two markets are not significantly different.
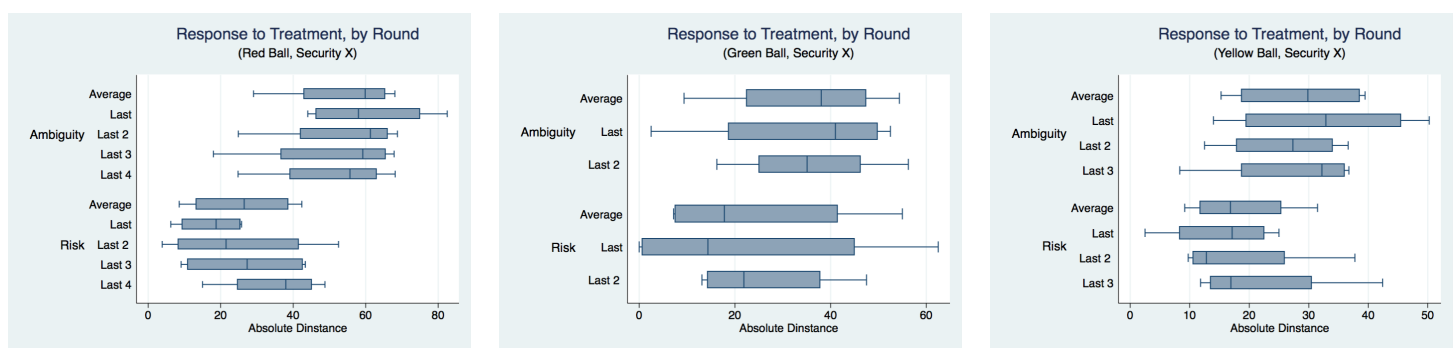

Figure 4.4: Box plots for treatments with security X. 
Similarly, in Figure 4.5 the box plots for the two treatments are shown, when the security is $Y$. There is no evidence for significant differences among markets. Hence, suggesting that our second hypothesis could be plausible as well.
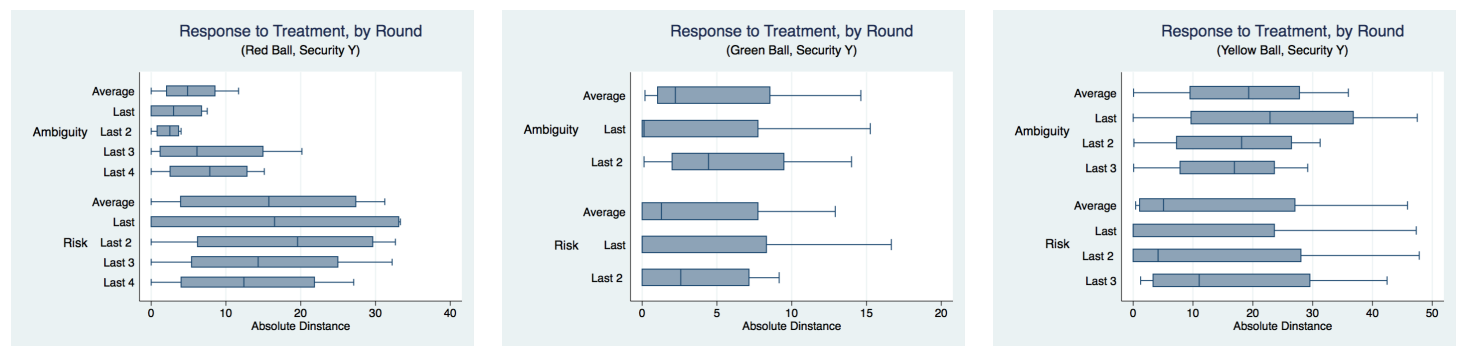

FiguRE 4.5: Box plots for treatments with security Y.

\subsubsection{Ambiguity Vs Risk for Security X}

In this subsection we report the results of treatments with the ambiguous and risky box when the $X$ is the traded security. In Figure 4.6 the p-values are illustrated. We used permutation tests in order to test the null hypothesis that the differences are due to chance.

\begin{tabular}{c|c|c|c}
\hline \multicolumn{2}{c}{ Security X } & Alternative Hypothesis & p-values \\
\hline \multirow{5}{*}{ Red Ball } & Last Round & Risk < Ambiguity & $\mathbf{0 . 0 1 4}$ \\
& Last 2 Rounds & Risk < Ambiguity & $\mathbf{0 . 0 4 3}$ \\
& Last 3 Rounds & Risk < Ambiguity & $\mathbf{0 . 0 5 7}$ \\
& Last 4 Rounds & Risk $\neq$ Ambiguity & 0.227 \\
& Average & Risk < Ambiguity & $\mathbf{0 . 0 4 2}$ \\
\hline \multirow{5}{*}{ Green Ball } & Last Round & Risk $\neq$ Ambiguity & 0.601 \\
& Last 2 Rounds & Risk $\neq$ Ambiguity & 0.428 \\
& Last 3 Rounds & Risk $\neq$ Ambiguity & 0.572 \\
& Average & Risk $\neq$ Ambiguity & 0.457 \\
\hline \multirow{5}{*}{ Yellow Ball } & Last Round & Risk $\neq$ Ambiguity & 0.201 \\
& Last 2 Rounds & Risk $\neq$ Ambiguity & 0.391 \\
& Last 3 Rounds & Risk $\neq$ Ambiguity & 0.57 \\
& Average & Risk < Ambiguity & 0.1 \\
\hline
\end{tabular}

Figure 4.6: Permutation tests for treatments with security X.

When the ball is red the AD in the ambiguous market is higher than in the respective risky market. We can observe that focusing on the later rounds the difference is getting more and more significant, and the average data suggest significance at $5 \%$ level. In fact, for the final round we get the most significance we can, given the constraint that we only have four independent observations.

The analysis for green ball is unambiguous too. They suggest that there is no significant difference, for either focusing on the 3 final rounds or the latter ones. Finally, when the ball is yellow, the result indicate strong insignificant difference for the 3 and 2 last rounds. Therefore, although the final round the result turns to be less insignificant it is still in the right direction.

The results suggest that ambiguity has an effect on information aggregation when the security is $X$. In other words, ambiguity aversion potentially is an extra layer that 
complicates for a trader to incorporate the signal of the other trader's prediction, in case of security $X$. All in all, these results confirm the theoretical results of Chapter 2 and Chapter 3 for all treatments and in particular our first hypothesis.

\subsubsection{Ambiguity Vs Risk for Security Y}

In this subsection we report the results of permutation tests for the treatments with security $Y$. In particular, they are shown in Figure 4.7.

We can conclude that we get strong evidence that there is no significant difference among the two markets. In fact, two markets are very similar in terms of the accurateness of traders' predictions. The only significant difference that we observe is for red balls when re restrict to the last two rounds. As it is shown in Appendix A, Figure 14, in the treatment with ambiguity and security $Y$ in the round before the last round in which a red ball was drawn, the prediction are getting unevenly accurate. However, given that this is observed only on that round and given how easily our results are affected even for small mistakes by participants (due to the small number of independent observations), we can conclude that significantly there is not difference between the two markets.

\begin{tabular}{|c|c|c|c|}
\hline \multicolumn{2}{|c|}{ Security $Y$} & Alternative Hypothesis & p-values \\
\hline Red Ball & \begin{tabular}{|l} 
Last Round \\
Last 2 Rounds \\
Last 3 Rounds \\
Last 4 Rounds \\
Average
\end{tabular} & $\begin{array}{l}\text { Risk } \neq \text { Ambiguity } \\
\text { Risk }<\text { Ambiguity } \\
\text { Risk } \neq \text { Ambiguity } \\
\text { Risk } \neq \text { Ambiguity } \\
\text { Risk } \neq \text { Ambiguity }\end{array}$ & $\begin{array}{c}0.4268 \\
\mathbf{0 . 0 7 1} \\
0.48375 \\
0.48703 \\
0.283\end{array}$ \\
\hline Green Ball & $\begin{array}{l}\text { Last Round } \\
\text { Last } 2 \text { Rounds } \\
\text { Last } 3 \text { Rounds } \\
\text { Average }\end{array}$ & $\begin{array}{l}\text { Risk } \neq \text { Ambiguity } \\
\text { Risk } \neq \text { Ambiguity } \\
\text { Risk } \neq \text { Ambiguity } \\
\text { Risk } \neq \text { Ambiguity }\end{array}$ & $\begin{array}{c}1 \\
0.6867 \\
0.485 \\
0.6002\end{array}$ \\
\hline Yellow Ball & $\begin{array}{l}\text { Last Round } \\
\text { Last } 2 \text { Rounds } \\
\text { Last } 3 \text { Rounds } \\
\text { Average }\end{array}$ & $\begin{array}{l}\text { Risk } \neq \text { Ambiguity } \\
\text { Risk } \neq \text { Ambiguity } \\
\text { Risk } \neq \text { Ambiguity } \\
\text { Risk } \neq \text { Ambiguity }\end{array}$ & $\begin{array}{l}0.5486 \\
0.8571 \\
0.9152 \\
0.7131\end{array}$ \\
\hline
\end{tabular}

FIGURE 4.7: Permutation tests for treatments with security Y.

These results suggest that, as opposed to security $X$, security $Y$ is robust enough in order traders to be able to interactively update their beliefs, concluding the realised state. Hence, information aggregation is not affected by ambiguity, therefore confirming the results in Chapter 2 and Chapter 3, hence our second hypothesis.

\subsubsection{Results with Learning Effects}

In this subsection the effects of learning, through the course of the periods, are analysed. In particular, for each treatment, we take the average $\mathrm{AD}$, across all sessions, for each round. Then, we group appropriately the average data for each round in which the same colour has been drawn. In fact, for each colour, we create a subgroup containing the final two rounds' average data, and a second subgroup with the rest. For robustness purposes, we use a modified protocol to group the average data. In particular, the first 
subgroup contains the final three rounds' average data and the second subgroup contains the rest.

For each case, we test for the existence of learning effects by running permutation test using the data of the two groups. We test the null hypothesis that the two groups have the same AD. Figure 4.8 illustrates the results of the tests for security $X$.

\begin{tabular}{|c|c|c|c|c|}
\hline \multicolumn{5}{|c|}{ Learning effects } \\
\hline & & Red Balls & Green Balls & Yellow Balls \\
\hline \multirow[t]{2}{*}{ Risk } & Last 3 Rounds & $2.79 \%$ & $4.58 \%$ & $2.80 \%$ \\
\hline & Last 2 Rounds & $9.53 \%$ & $13.35 \%$ & $4.70 \%$ \\
\hline \multirow[t]{2}{*}{ Ambiguity } & Last 3 Rounds & $57.43 \%$ & $4.89 \%$ & $17.00 \%$ \\
\hline & Last 2 Rounds & $33.19 \%$ & $6.71 \%$ & $32.19 \%$ \\
\hline
\end{tabular}

FiguRE 4.8: Learning patterns for treatments with security X.

We can observe that for the risk treatment with security $X$ there is a consistent trend of learning across the various realised states. The final 3 rounds are significantly different than the previous. Given that, in our main statistical analysis we will use the data of the final 3 rounds only. On the other hand, in the treatment with ambiguity only for green ball we can observe a significant effect of learning. Hence, for the ambiguity treatment with security $X$, in case the ball is green we will use the data of the final three rounds, while in case the ball is red or yellow we will use the data of all rounds.

We report the respective analysis for security $Y$ in the Figure 4.9. No significant patterns of learning exist, except in the ambiguity treatment when ball is red. As before, in our main statistical analysis we will use the final two periods' average data for the red ball case.

\begin{tabular}{|c|c|c|c|c|}
\hline \multicolumn{5}{|c|}{ Learning effects } \\
\hline & & Red Balls & Green Balls & Yellow Balls \\
\hline \multirow[t]{2}{*}{ Risk } & \multirow{2}{*}{$\begin{array}{l}\text { Last } 3 \text { Rounds } \\
\text { Last } 2 \text { Rounds }\end{array}$} & $11.41 \%$ & $15.00 \%$ & $15.09 \%$ \\
\hline & & $19.22 \%$ & $53.00 \%$ & $53.30 \%$ \\
\hline \multirow[t]{2}{*}{ Ambiguity } & Last 3 Rounds & $77.16 \%$ & $29.67 \%$ & $30.07 \%$ \\
\hline & Last 2 Rounds & $4.84 \%$ & $46.64 \%$ & $46.57 \%$ \\
\hline
\end{tabular}

FigURE 4.9: Learning patterns for treatments with security X.

In Figure 4.10, the boxplots are given across treatments and colours. We can observe that except for the treatments with security $X$ when the ball is red, in which we can observe a clear difference, the rest boxplots do not suggest significant differences.

\subsubsection{Ambiguity Vs Risk for Security X}

In Figure 4.11, the results of the permutation tests, for the treatment with security $X$, are reported. The only case that we can significantly, at $5 \%$ level, reject the null 

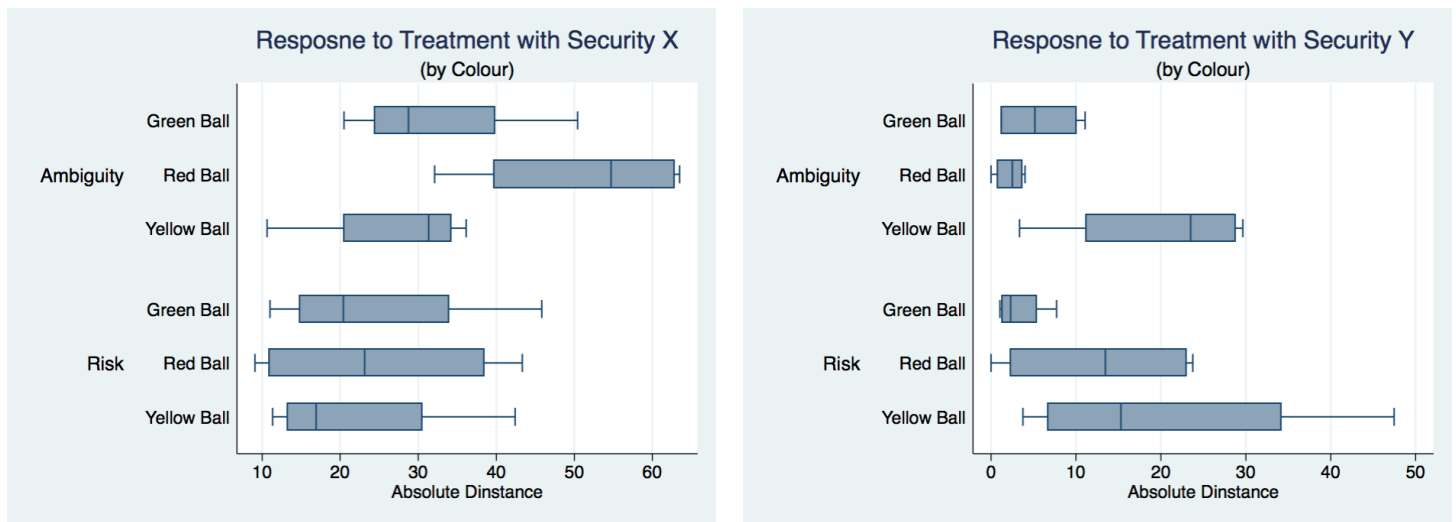

FigURE 4.10: Box plots incorporating learning effects.

hypothesis in favor of the alternative is when the ball is red. The other two cases do not suggest statistically significant differences between the market with ambiguity and risk.

These results are consistent with the respective in Section 4.5.1.1 hence suggesting more evidence in our first hypothesis.

\begin{tabular}{|c|c|c|}
\hline Security $\mathrm{X}$ & Alternative Hypothesis & p-values \\
\hline Red Ball & Risk < Ambiguity & 0.041 \\
\hline Gren Ball & Risk $\neq$ Ambiguity & 0.429 \\
\hline Yellow Ball & Risk $\neq$ Ambiguity & 0.513 \\
\hline
\end{tabular}

Figure 4.11: Permutation tests for treatments with security X.

\subsubsection{Ambiguity Vs Risk for Security Y}

In Figure 4.12, the results of the permutation tests for the treatment with security $Y$. We can observe that the results do not suggest significant differences between the markets with ambiguity and risk. On the contrary to section 4.5.2.1, there is evidence that security $Y$ overcomes the effect of ambiguity, which provides more evidence for our second hypothesis.

\begin{tabular}{|c|c|c|}
\hline Security $X$ & Alternative Hypothesis & $p$-values \\
\hline Red Ball & Risk $\neq$ Ambiguity & 0.229 \\
\hline Gren Ball & Risk $\neq$ Ambiguity & 0.484 \\
\hline Yellow Ball & Risk $\neq$ Ambiguity & 0.94 \\
\hline
\end{tabular}

Figure 4.12: Permutation tests for treatments with security X.

We can observe that the p-value when the ball is red is not large enough, although suggesting insignificance. This is because the learning effect indicated that we need to restrict to data of the final two rounds only. Taking into consideration the p-value in Figure 4.7 when the ball is red and when we restrict to the last two rounds, and the rest p-values when the ball is red, we can conclude that the round before the last one (when the ball is red) the predictions in ambiguity treatment are sharply more accurate. Hence we can interpret the p-value of Figure 4.12 when the ball is red as the one based on the 
most accurate predictions given by trades in the ambiguity treatment. This perspective argues, in turn, that the result is robust.

\subsection{Conclusion}

This chapter studies information aggregation in a dynamic market under ambiguity. We find evidence in favor of the theoretical results of Chapter 2 and 3.

In particular, when an Arrow-Debreu security, is traded in the market, we find evidence that its price is significantly less accurate in case the fundamentals of the security are ambiguous. On the contrary, when introducing in the market a "separable under ambiguity" security, making insider one of the two traders, we find evidence that ambiguity about the fundamentals does not affect market's degree of information aggregation.

This chapter leaves out some interesting questions for future research. In fact, robustness checks should be carried out in order to understand fully the dynamics of the ambiguity effect. Finally, more session can be ran in order to have more independent observations. 



\section{Chapter 5}

\section{Conclussion}

This Thesis contributes to the theoretical and experimental literature on information aggregation. By relaxing the subjective expected utility assumption in MSR markets, it studies the effect of ambiguity on the efficiency of information aggregation.

In Chapter 2, the class of separable under ambiguity securities was defined. It was proved that it is a strict subset of separable under SEU securities, which is the class that characterises information aggregation under subjective expected utility assumptions. Through counterexamples, it was observed that under ambiguity separable under $S E U$ securities do not characterise information aggregation. Hence, a new result was provided, restricting to myopic traders, which characterises information aggregation in terms of the class of separable under ambiguity securities.

In Chapter 3, the assumption of myopic traders was relaxed, allowing for strategic interactions. Hence, a new equilibrium concept was defined. This concept accommodates time inconsistency, infinite horizon and incomplete information. The main result stated that in every pure equilibrium information gets aggregated if and only if the security of the market is separable under ambiguity. This chapter contributes both to the literature of game theory under ambiguity and information aggregation under ambiguity. In addition, two results were provided stating the conditions under which strategic behavior is myopic.

Finally, Chapter 5 applies the theoretical model to a laboratory experiment. Two main hypotheses were tested, providing evidence in favor of the theoretical predictions of Chapter 2 and Chapter 3. On the one hand, ambiguity distorted the information revelation within the participants. On the other hand, the design of separable under ambiguity precluded these distortions and allowed information to be communicated smoothly in the market.

Each chapter leaves some interesting question to be answered in future work. In this thesis we have not analysed what happens when new traders starts trading entering 
the market randomly. In addition, if the turn that traders predict in the market is random, does it affect how information gets aggregated ? Moreover, can the assumption of converging strategies be avoided? Does an MSR game have an equilibrium? Finally, robustness checks should be carried out in order to understand fully the dynamics of the ambiguity effect in our experimental paper and more sessions can be ran in order to have more independent observations. 


\section{Appendix A}

Here is the proof of Lemma 2.3: Where convenient we use the notation $s(y)(.) \equiv$ $s(y, X()$.$) , where X$ is the security.

Proof. Let's first prove, that the $\underset{y \in Y}{\operatorname{argax}} \min _{p \in \mathcal{P}} E_{p}\left[s(y)-s\left(y_{-1}\right)\right]$ does, in fact, exist. This is true because $s$ is continuous function, therefore $\min _{p \in \mathcal{P}} E_{p}\left[s(y)-s\left(y_{-1}\right)\right]$ is upper semi continuous (as infimum of continuous functions) as a function of $y$. As $Y$ is compact

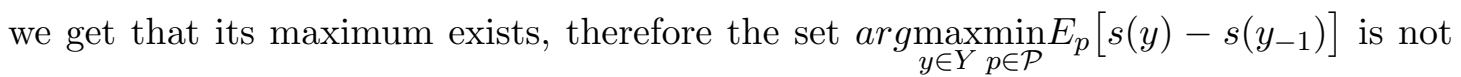
empty.

Next, we define $Z$ to be the convex hull of $\{s(y)\}_{y \in Y}$. The set $\{s(y)\}_{y \in Y}$ is compact in $\mathbb{R}^{l}$ because $s$ is continuous in $y$ and $Y$ is compact, hence $Z$ is compact. Consider the function $G: \mathcal{P} \times Z \longrightarrow \mathbb{R}$ defined by $G(p, z)=E_{p}\left[z-s\left(y_{-1}\right)\right]$. The function is linear in $p$ and affine in $z$. Moreover, it is continuous both in $p$ and in $z$. The first is because of the definition of weak* convergence and the second applying Lebesgue's dominated convergence theorem.

Then by Sion minimax Theorem, in Berge (1963) (p. 210), there exists $p^{*} \in \mathcal{P}$ and $z^{*} \in Z$ such that for all $(p, z) \in \mathcal{P} \times Z$ it is:

$$
E_{p^{*}}\left[z-s\left(y_{-1}\right)\right] \leq E_{p^{*}}\left[z^{*}-s\left(y_{-1}\right)\right] \leq E_{p}\left[z^{*}-s\left(y_{-1}\right)\right]
$$

Then we get that

$$
\operatorname{minmax}_{p \in \mathcal{P}} E_{z \in Z}\left[z-s\left(y_{-1}\right)\right]=\operatorname{maxmin}_{z \in Z} E_{p \in \mathcal{P}}\left[z-s\left(y_{-1}\right)\right]
$$

and it is achieved at $p=p^{*}, z=z^{*}$.

For a fixed $p$, and because $G(p, z)$ is affine in $z$, the unique maximiser of $E_{p}\left[z-s\left(y_{-1}\right)\right]$ over $z$ is $s\left(E_{p}[X]\right)$ ( as $s$ is a proper scoring rule, by definition of Z), so that $z^{*}=$ 
$s\left(E_{p^{*}}[X]\right)$. Hence we may conclude

$$
\operatorname{minmax}_{p \in \mathcal{P}} E_{y \in Y}\left[s(y)-s\left(y_{-1}\right)\right]=\operatorname{maxmin}_{y \in Y} E_{p \in \mathcal{P}}\left[s(y)-s\left(y_{-1}\right)\right]
$$

and it is achieved at $p=p^{*}, y=E_{p^{*}}[X]$.

We claim that $y=E_{p^{*}}[X]$ is a unique element of $\underset{y \in Y}{\operatorname{argmaxmin}} E_{p \in \mathcal{P}}\left[s(y, X(\omega))-s\left(y_{-1}, X(\omega)\right)\right]$.

To see that, let $y^{\prime} \neq E_{p^{*}}[X]$. Then:

$$
\begin{gathered}
\min _{p \in \mathcal{P}} E_{p}\left[s\left(y^{\prime}, X(\omega)\right)-s\left(y_{-1}, X(\omega)\right)\right] \leq \\
E_{p^{*}}\left[s\left(y^{\prime}, X(\omega)\right)-s\left(y_{-1}, X(\omega)\right)\right]< \\
E_{p^{*}}\left[s\left(E_{p^{*}}[X], X(\omega)\right)-s\left(y_{-1}, X(\omega)\right)\right]= \\
\operatorname{maxmin}_{y \in Y} E_{p \in \mathcal{P}}\left[s(y, X(\omega))-s\left(y_{-1}, X(\omega)\right)\right]
\end{gathered}
$$

Hence, the maximiser is unique.

Now we proceed with two technical results which we need for later steps.

Lemma A.1. Let $\mathcal{P} \subseteq \Delta(\Omega)$ be a set of priors and a subset $A \subseteq \Omega$ with $p(A)>0$ for all $p \in \mathcal{P}$. Define $C^{*}=\{c(\cdot)=p(\cdot \mid A): p \in \mathcal{P}\}$. Then $d_{\mathcal{P}}\left(\Pi_{i}(\omega) \cap A, z\right)=d_{\mathcal{C}^{*}}\left(\Pi_{i}(\omega), z\right)$, for every trader $i$ and every given prediction $z \in \mathbb{R}$.

Proof. We can notice that for every $y \in Y$ it is

$$
\begin{gathered}
\min _{p \in \mathcal{P}} E_{p_{\mid A \cap \Pi_{i}(\omega)}}[s(y, X)-s(z, X)]=E_{p_{\mid A \cap \Pi_{i}(\omega)}^{\prime}}[s(y, X)-s(z, X)]= \\
E_{c_{\mid \Pi_{i}(\omega)}^{\prime}}[s(y, X)-s(z, X)] \geq \min _{c \in C^{*}} E_{c_{\mid \Pi_{i}(\omega)}}[s(y, X)-s(z, X)] .
\end{gathered}
$$

Similarly, for every $y \in Y$ it is

$$
\begin{aligned}
& \min _{c \in C^{*}} E_{c \mid \Pi_{i}(\omega)}[s(y, X)-s(z, X)]=E_{c_{\mid \Pi_{i}(\omega)}^{\prime \prime}}[s(y, X)-s(z, X)]= \\
& E_{p_{\mid A \cap \Pi_{i}(\omega)}^{\prime \prime}}[s(y, X)-s(z, X)] \geq \min _{p \in \mathcal{P}} E_{p_{\mid A \cap \Pi_{i}(\omega)}}[s(y, X)-s(z, X)] .
\end{aligned}
$$

Hence we get the result. 
Here is the proof of Lemma 2.8:

Proof. (i) Consider $\mathcal{P} \subseteq \Delta(\Omega)$ convex and compact set of priors over $\Omega$ and $y_{0}$ the initial prediction of the market maker. Every trader knows that everybody knows that... $\Pi_{i}$ is each trader's partition and all the public announcements made in the market. Let $\Pi_{i, t}(\omega)$ be the partition cell of trader $i$ at time $t$, before the $t-t h$ announcement made in the market.

At $t=1$, trader 1 makes a myopic prediction, hence her announcement is $y_{1}=$ $d_{\mathcal{P}}\left(\Pi_{1}(\omega), y_{0}\right)$. The outside observer whose information cell, when true state is $\omega$, was initially $\mathcal{F}_{0}(\omega)=\left\{\omega^{\prime} \in \Omega: p\left(\omega^{\prime}\right)>0\right.$ for some $\left.p \in \mathcal{P}\right\}$, after the first prediction it is

$$
\mathcal{F}_{1}\left(\omega, y_{0}\right)=\left\{\omega^{\prime} \in \mathcal{F}_{0}(\omega): d_{\mathcal{P}}\left(\Pi_{1}\left(\omega^{\prime}\right), y_{0}\right)=y_{1}\right\}
$$

Then each trader refines her own information as well, hence at time $t$ the prediction of $t \bmod n$ trader is

$$
y_{t}=d_{\mathcal{P}}\left(\Pi_{t \operatorname{modn}}(\omega) \cap \mathcal{F}_{t-1}\left(\omega, y_{t-2}\right), y_{t-1}\right)
$$

with $\mathcal{F}_{t}\left(\omega, y_{t-1}\right)=\left\{\omega^{\prime} \in \mathcal{F}_{t-1}\left(\omega, y_{t-2}\right): d_{\mathcal{P}}\left(\Pi_{t m o d n}\left(\omega^{\prime}\right) \cap \mathcal{F}_{t-1}\left(\omega, y_{t-2}\right), y_{t-1}\right)=y_{t}\right\}$.

It is obvious that $\mathcal{F}_{0}(\omega) \supseteq \mathcal{F}_{1}\left(\omega, v_{0}\right) \supseteq \ldots \supseteq \mathcal{F}_{t}\left(\omega, y_{t-1}\right)$. Because $\Omega$ is finite, there exists $t^{\star}$ such that $\mathcal{F}_{t}\left(\omega, y_{t-1}\right)=\mathcal{F}_{t^{\star}}\left(\omega, y_{t^{\star}-1}\right)$ for every $t \geq t^{\star}$. Let assume, without loss in generality that $t^{\star}$ is the time of the first player in round $T^{\star}$.

(ii) Fix $z \in \mathbb{R}$, interpreted as the previous trader's prediction. Firstly, we can observe that the function $\Phi(p)=E_{p}\left[s\left(E_{p}[X], X\right)-s(z, X)\right]$, with $p \in \Delta(\Omega)$ is convex in $p$, for any $z \in[a, b]$, with $a=\min \left\{E_{p}[X]: p \in \Delta(\Omega)\right\}$ and $b=\max \left\{E_{p}[X]: p \in \Delta(\Omega)\right\}$. Define the function $g\left(E_{p}[X]\right)=\Phi(p)$. We can see that $g$ is convex in $\left\{E_{p}[X]: p \in \Delta(\Omega)\right\}$ and hence because has a unique minimiser which turns out to be $z$ we get that $g$ is decreasing at $[a, z]$ and increasing at $[z, b]{ }^{30}$ We can observe that the myopic announcement of trader $i$, when the previous is $z$, is given by $d_{\mathcal{P}_{\mathcal{F}_{t}(\omega)}}\left(\Pi_{i}(\omega), z\right)=E_{p^{*}}[X]$ for some $p^{*} \in$

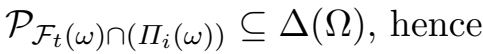

$$
d_{\mathcal{P}_{\mathcal{F}_{t}(\omega)}}\left(\Pi_{i}(\omega), z\right)=\arg \min _{x \in\left\{E_{p}[X]: p \in \mathcal{P}_{\left.\mathcal{F}_{t}(\omega) \cap\left(\Pi_{i}(\omega)\right)\right\}}\right.} g(x)
$$

In fact, if $z<x$ for every $x \in\left\{E_{p}[X]: p \in \mathcal{P}_{\mathcal{F}_{t}(\omega) \cap\left(\Pi_{i}(\omega)\right)}\right\}$ then the lowest extreme point of the interval $\left\{E_{p}[X]: p \in \mathcal{P}_{\mathcal{F}_{t}(\omega) \cap\left(\Pi_{i}(\omega)\right)}\right\}$ is the minimising value, and the greatest extreme point in case $z>x$ for every $x \in\left\{E_{p}[X]: p \in \mathcal{P}_{\mathcal{F}_{t}(\omega) \cap\left(\Pi_{i}(\omega)\right)}\right\}$ (because of the

\footnotetext{
${ }^{30}$ We can observe that there exists $p \in \Delta(\Omega)$ such that $E_{p}[X]=z$. In addition, the set $\left\{E_{p}[X]: p \in \mathcal{P}\right\}$ is interval, as convex and closed set of the real numbers.
} 
convexity of $\Phi$, hence of $g$ and the fact that $z$ is global minimum). ${ }^{31}$

\section{Step 1:}

Define $A_{\omega^{\prime}}^{i}=\left\{E_{p}\left[X \mid \Pi_{i}\left(\omega^{\prime}\right)\right]: p \in \mathcal{P}_{\mathcal{F}_{t^{\star}}\left(\omega, y_{t^{\star}-1}\right)}\right\}$ for every $i=1, \ldots, n$ and $\omega^{\prime} \in$ $\mathcal{F}_{t^{\star}}\left(\omega, y_{t^{\star}-1}\right)=\left\{\omega_{k_{1}}, \ldots, \omega_{k_{l}}\right\}$. Because, by $(i)$, the public information, $\mathcal{F}_{t^{\star}}\left(\omega, y_{t^{\star}-1}\right)$, has reached its finest form, there is no information revelation in the market

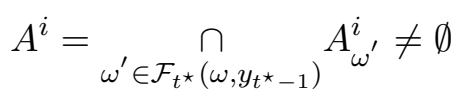

for every $i=1, \ldots, n$.

To prove that claim, assume there exists $i$ such that $A^{i}=\emptyset$. Define

$$
k_{\lambda}=\min \left\{k_{i}: \underset{\omega^{\prime} \in\left\{\omega_{k_{1}}, \ldots, \omega_{k_{i}}\right\}}{\cap} A_{\omega^{\prime}}^{i} \neq \emptyset\right\} .
$$

Because $A_{\omega_{k_{\lambda}}}^{i}$ and $\underset{\omega^{\prime} \in\left\{\omega_{k_{1}}, \ldots, \omega_{k_{\lambda}-1}\right\}}{\cap} A_{\omega}^{i}$ have empty intersection, by definition of $k_{\lambda}$, and both are intervals assume, without loss in generality, that for every $a \in A_{\omega_{\lambda}}^{i}$ and every $a^{\prime} \in \underset{\omega^{\prime} \in\left\{\omega_{k_{1}}, \ldots, \omega_{k_{\lambda}-1}\right\}}{\cap} A_{\omega}^{i}$ it is $a<a^{\prime}$. We can observe, as well, that the smallest extreme point of $\cap_{\omega^{\prime} \in\left\{\omega_{k_{1}}, \ldots, \omega_{k_{\lambda}-1}\right\}} A_{\omega^{\prime}}^{i}$ is the smallest extreme point of $A_{\omega_{k}}^{i}$ for some $\omega_{k} \in\left\{\omega_{k_{1}}, \ldots, \omega_{k_{\lambda}-1}\right\}$. Therefore, for any announcement of $(i-1)$-th trader, the candidate predictions, of trader $i$, at state $\omega_{k_{\lambda}}$ and $\omega_{k}$ are different, hence there should be information revelation. And hence we get a contradiction.

Similarly, we can conclude that in order no information to be revealed, the announcement of each trader $i$ (which is the same for every state in $\mathcal{F}_{t^{\star}}\left(\omega, y_{t^{\star}-1}\right)$ ) should lie in the interval $A^{i}$ (which we know it is non empty by the previous claim). To prove it, assume again that the announcement $y \notin A^{i}$ for some trader $i$, and in particular, without loss in generality we assume $y<b$ for every $b \in A^{i}$. Hence, as before, there is $\omega_{m} \in \mathcal{F}_{t^{\star}}\left(\omega, y_{t^{\star}-1}\right)$ such that the smallest extreme point of $A_{\omega_{m}}^{i}$ is the same as the smallest extreme point of $A^{i}$, and therefore the announcement of $i$ at $\omega_{m}$ is different than $y$ (for any announcement of $(i-1)$-th trader). Hence we have revelation of information and hence we get the contradiction.

\section{Step 2:}

Case $\underset{j \in\{1, \ldots, n\}}{\cap} A^{j}=\emptyset$

Define $i_{0}=\min \left\{i: \underset{j \in\{1, \ldots, i\}}{\cap} A^{j}=\emptyset\right\}$. Therefore, $A^{i_{0}}$ has empty intersection with

\footnotetext{
${ }^{31}$ The definition of extreme point that we use here is the following: An extreme point of a convex set, $C$, is a point $x_{1} \in C$, with the property that if $x_{1}=k x_{2}+(1-k) x_{3}$ with $x_{2}, x_{3} \in C$ and $k \in[0,1]$, then $x_{1}=x_{2}$ and $/$ or $x_{1}=x_{3}$
} 
$\cap_{j \in\left\{1, \ldots, i_{0}-1\right\}} A^{j}$, and without loss in generality we assume that for every $d \in A^{i_{0}}$ and every $d^{\prime} \in \underset{j \in\left\{1, \ldots, i_{0}-1\right\}}{\cap} A^{j}$ it is $d<d^{\prime}$. Because $\underset{j \in\left\{1, \ldots, i_{0}-1\right\}}{\cap} A^{j}$ is an interval we can conclude that there are $A^{i_{1}}$ and $A^{i_{2}}$ such that one of them defines the smallest extreme point of the interval and the other one greatest extreme point.

We can observe that for any value that $y_{t_{0}-1}$ might have, either trader $i_{1}$ or $i_{2}$ (or both) gives a prediction belonging in the set $\cap_{j \in\left\{1, \ldots, i_{0}-1\right\}} A^{j}$. By the definition of the function $g$ we get that from trader $\max \left\{i_{1}, i_{2}\right\}$ until trader $i_{0}-1$ the corresponding prediction belongs, as well, to $\cap_{j \in\left\{1, \ldots, i_{0}-1\right\}} A^{j}$. Hence, always the prediction of $i_{0}$ is the greatest extreme point of $A^{i_{0}}$, and let's denote it by $v_{i_{0}}$. We denote the announcements of traders $j=i_{0}+1, \ldots, n$ with $v_{j}$.

For the next round, the $v_{n}$ potentially triggers different announcements for traders $j=1, \ldots, i_{0}$ (compared to their announcements of the previous period) and denote them by $v_{j}$. But, with exactly the same argument as before, the announcement of $i_{0}-1$ should belong to $\cap_{j \in\left\{1, \ldots, i_{0}-1\right\}} A^{j}$. Hence, the announcements of traders $i_{0}, \ldots, n$ remain $v_{i_{0}}, \ldots, v_{n}$, and hence the $v_{1}, \ldots, v_{n}$ remain the same for any later round. Hence we get the conclusion.

\section{Case $\underset{j \in\{1, \ldots, n\}}{\cap} A^{j} \neq \emptyset$}

As before, there are $A^{i_{1}}$ and $A^{i_{2}}$ such that one of them defines the smallest extreme point of the interval and the other one the greatest extreme point. Hence for any value of the $y_{t_{0}-1}$ either trader $i_{1}$ or $i_{2}$ (and in particular trader $i_{3}=\max \left\{i_{1}, i_{2}\right\}$ ) gives a prediction belonging in the set $\bigcap_{j \in\{1, \ldots, n\}} A^{j}$. We denote the corresponding announcement with $v_{i_{3}}$. By definition of $g$ we conclude that for $j=i_{3}, \ldots, n$ their announcements are $v_{i_{3}}$. Because $v_{i_{3}} \in \underset{j \in\{1, \ldots, n\}}{\cap} A^{j}$ we conclude that at the next round the announcement of each trader $1, \ldots, i_{3}-1$ is $v_{i_{3}}$, too. Hence we get $v_{1}=\ldots=v_{n}=v_{i_{3}}$.

(iii) Denote, for simplicity, $\mathcal{F}_{t^{\star}}\left(\omega, y_{t^{\star}-1}\right)=\mathcal{F}_{T^{\star}}$. By $($ ii $)$ it is

$$
\min _{p \in \mathcal{P}} E_{p \mid \mathcal{F}_{T^{\star}} \cap \Pi_{i}\left(\omega^{\prime}\right)}\left[s\left(v_{i}, X\right)-s\left(v_{i-1}, X\right)\right] \geq 0
$$

for every $\omega^{\prime} \in \mathcal{F}_{T^{\star}}$, for every $i=1, \ldots, n .^{32}$

Therefore, for every $p \in \mathcal{P}$ it is

$$
p\left(\mathcal{F}_{T^{\star}} \cap \Pi_{i}\left(\omega^{\prime}\right)\right) E_{p \mid \mathcal{F}_{T^{\star}} \cap \Pi_{i}\left(\omega^{\prime}\right)}\left[s\left(v_{i}, X\right)-s\left(v_{i-1}, X\right)\right] \geq 0
$$

\footnotetext{
${ }^{32} \mathrm{By} v_{0}$ we denote, when appropriate, the $v_{n}$.
} 
and $p\left(\mathcal{F}_{T^{\star}} \cap \Pi_{i}\left(\omega^{\prime}\right)\right)>0$, for every $\omega^{\prime} \in \mathcal{F}_{T^{\star}}{ }^{33}$ Summing over $\mathcal{C}_{i}=\left\{\Pi_{i}(\omega): \omega \in \mathcal{F}_{T^{\star}}\right\}$ we get

$$
p\left(\mathcal{F}_{T^{\star}}\right) E_{p_{\mid \mathcal{F}_{T^{\star}}}}\left[s\left(v_{i}, X\right)-s\left(v_{i-1}, X\right)\right] \geq 0
$$

, for every $i=1, \ldots, n$.

Then we sum over $i$, ignoring $p\left(\mathcal{F}_{T^{\star}}\right)$ :

$E_{p \mid \mathcal{F}_{T^{\star}}}\left[s\left(v_{1}, X\right)-s\left(v_{n}, X\right)\right]+E_{p \mid \mathcal{F}_{T^{\star}}}\left[s\left(v_{2}, X\right)-s\left(v_{1}, X\right)\right]+\ldots++E_{p \mid \mathcal{F}_{T^{\star}}}\left[s\left(v_{n}, X\right)-s\left(v_{n-1}, X\right)\right]=0$.

Because each term is non negative for every $p \in \mathcal{P}$ it should be

$$
E_{p \mid \mathcal{F}_{T^{\star}}}\left[s\left(v_{i}, X\right)-s\left(v_{i-1}, X\right)\right]=0
$$

for every $i=1, \ldots, n$. For the same reason,

$$
E_{p \mid \mathcal{F}_{T^{\star}} \cap \Pi_{i}\left(\omega^{\prime}\right)}\left[s\left(v_{1}, X\right)-s\left(v_{n}, X\right)\right]=0
$$

for every $\omega^{\prime} \in \mathcal{F}_{T^{\star}}$ and for every $p \in \mathcal{P}$.

Therefore, for every $i=1, \ldots, n$ it is

$$
\min _{p \in \mathcal{P}} E_{p \mid \mathcal{F}_{T^{\star}} \cap \Pi_{i}\left(\omega^{\prime}\right)}\left[s\left(v_{i}, X\right)-s\left(v_{i-1}, X\right)\right]=0
$$

for every $\omega^{\prime} \in \mathcal{F}_{T^{\star}}$. Hence,

$$
E_{p^{*} \mid \mathcal{F}_{T^{\star}} \cap \Pi_{i}\left(\omega^{*}\right)}\left[s\left(E_{p^{*} \mid \mathcal{F}_{T^{\star}}(\omega) \cap \Pi_{i}\left(\omega^{*}\right)}[X], X\right)-s\left(v_{i-1}, X\right)\right]=0
$$

for every $\omega^{\prime} \in \mathcal{F}_{T^{\star}}$, where $E_{p^{*} \mid \mathcal{F}_{T^{\star}} \cap \Pi_{i}\left(\omega^{*}\right)}[X]=d_{\mathcal{P}}\left(\Pi_{i}\left(\omega^{\prime}\right) \cap \mathcal{F}_{T^{\star}}, v_{i-1}\right)=v_{i}$. Because $s$ is strictly proper scoring rule, we get

$$
v_{i-1}=E_{p^{*} \mid \mathcal{F}_{T^{\star}} \cap \Pi_{i}\left(\omega^{\prime}\right)}[X]=d_{\mathcal{P}_{\mathcal{F}_{T^{\star}}}}\left(\Pi_{i}\left(\omega^{\prime}\right), v_{i-1}\right)=v_{i}
$$

for every $\omega^{\prime} \in \mathcal{F}_{T^{\star}}$ and every $i=1, \ldots, n$. Hence the agreement is concluded.

Here is the proof of Theorem 2.7:

Proof. $(\Leftarrow)$

Suppose $X$ is separable under ambiguity. By Lemma 2.8, we know that there exists time $t^{\star}$ such that $\mathcal{F}_{t}\left(\omega, y_{t-1}\right)=\mathcal{F}_{t^{\star}}\left(\omega, y_{t^{\star}-1}\right)$ for every $t \geq t^{\star}$. We denote this set by $\mathcal{F}_{t^{\star}}$.

\footnotetext{
${ }^{33}$ It is $p\left(\mathcal{F}_{T^{\star}} \cap \Pi_{i}\left(\omega^{\prime}\right)\right)>0$ for every $p \in \mathcal{P}$. This is because for every $\omega^{\prime} \in \mathcal{F}_{T^{\star}}$ there exists $p \in \mathcal{P}$ with $p\left(\omega^{\prime}\right)>0$, by its definition. We assumed the regularity property in Section ?? hence for every $p \in \mathcal{P}$ it is $p\left(\mathcal{F}_{T^{\star}} \cap \Pi_{i}\left(\omega^{\prime}\right)\right)$.
} 
Moreover, by Lemma $2.8(i i)$ and (iii) traders reach an agreement (at most within three rounds after $\left.t^{\star}\right)$, hence there exists $v \in \mathbb{R}$ such that for every $i=1, \ldots, n$ it is

$$
d_{\mathcal{P}}\left(\Pi_{i}(\omega) \cap \mathcal{F}_{t^{\star}}, v\right)=v
$$

for every $\omega \in \mathcal{F}_{t^{\star}}$, with $p\left(\omega \mid \mathcal{F}_{t^{\star}}\right)>0$ for some $p \in \mathcal{P}$ (this last property is trivially satisfied by construction of $\left.\mathcal{F}_{t}\left(\omega, y_{t-1}\right)\right)$. By defining $\mathcal{P}_{\mathcal{F}_{t^{\star}}}=\left\{p\left(\cdot \mid \mathcal{F}_{t^{\star}}\right): p \in \mathcal{P}\right\}$, we can observe, by Lemma A.1, that for every $i=1, \ldots, n$ it is $d_{\mathcal{P}_{\mathcal{F}^{\star}}}\left(\Pi_{i}(\omega), v\right)=v$ for every $\omega \in \Omega$, with $q(\omega)>0$ for some $q \in \mathcal{P}_{\mathcal{F}_{t^{\star}}}$.

By the definition of separability under ambiguity, with traders' ordering as selected, the set of priors $\mathcal{P}_{\mathcal{F}_{t^{\star}}}$, which is convex compact and mutually absolute continuous with respect to $\Pi_{i}$ for every $i=1, \ldots, n$, and the $v$ we observe that $(i i)$ is satisfied hence $(i)$ should be violated. Therefore, we get that $X(\omega)=k$ for some $k \in \mathbb{R}$ for every $\omega \in \Omega$, with $q(\omega)>0$ for some $q \in \mathcal{P}_{\mathcal{F}_{t^{\star}}}{ }^{34}$ Hence, information gets aggregated, because as soon as the security is constant for all states, for which at least one (updated) belief gives it strictly positive probability, and because by Lemma 1 the myopic prediction is an expectation using the appropriate (updated) belief, we conclude that the myopic predictions, which all are $v$, are just the constant value of the security.

$(\Rightarrow)$

Suppose information is aggregated for a given continuous strictly proper scoring rule $s$, and every $y_{0}$, traders' ordering $\mathcal{O}$ and $\mathcal{P} \in \mathcal{A}_{s, y_{0}, \mathcal{O}}$. This means:

$$
y_{t}(\omega)=d_{\mathcal{P}}\left(\Pi_{t m o d n}(\omega) \cap \mathcal{F}_{t-1}\left(\omega, y_{t-2}\right), y_{t-1}\right) \longrightarrow X(\omega)
$$

, for every $\omega \in \Omega$ with $p(\omega)>0$ for some $p \in \mathcal{P}$.

Following Chen et al. (2012), assume that hypothesis (ii) of Definition 2.6 is satisfied and we will show that $(i)$ is violated. ${ }^{35}$ Hence let $\mathcal{P} \subseteq \Delta(\Omega)$, convex, compact and mutually absolute continuous with respect to $\Pi_{i}$, for every $i=1, \ldots, n$, let $\mathcal{O}$ ordering and $v \in \mathbb{R}$ and assume that for every $i=1, \ldots, n$ it is $d_{\mathcal{P}}\left(\Pi_{i}(\omega), v\right)=v$, every $\omega$, with $p(\omega)>0$ for some $p \in \mathcal{P}$. We can now observe the following:

Pick for initial announcement $v \in \mathbb{R}$. We can observe that the predictions $y_{t}(\omega), t=$ $0,1, \ldots$, are constant across $\omega$, with $p(\omega)>0$ for some $p \in \mathcal{P}$, and in particular will be $v$ always.

\footnotetext{
${ }^{34}$ The absolute continuity is true because for an arbitrary $\omega \in \Omega$ and $i \in\{1, \ldots, n\}$ the $\Pi_{i}(\omega) \cap \mathcal{F}_{t^{\star}}$ is either an empty set or not. If it is empty, by definition of the set of priors, we get that all priors in $\mathcal{P}_{\mathcal{F}_{t^{\star}}}$ give zero probability. If it is not empty and if $\omega^{\prime} \in \Pi_{i}(\omega) \cap \mathcal{F}_{t^{\star}}$, then (by the definition of $\mathcal{F}_{t^{\star}}$ ) it can be realised as true state and because $\omega^{\prime} \in \mathcal{F}_{t^{\star}}$ every prediction would be the same hence it is $\mathcal{F}_{t^{\star}}=\mathcal{F}_{t^{\star}}\left(\omega^{\prime}, y_{t^{\star}-1}\right)$ and hence by the regularity assumption we get that the finest information set of the trader, $\mathcal{F}_{t^{\star}} \cap \Pi_{i}\left(\omega^{\prime}\right)$, has strictly positive probability for every prior, and hence the likelihood of $\Pi_{i}(\omega)=\Pi_{i}\left(\omega^{\prime}\right)$ is strictly positive for every prior.

${ }^{35}$ Assuming $(i)$ is satisfied and $(i i)$ is violated is an equivalent way to prove the theorem.
} 
Therefore, we can conclude that

$$
\mathcal{F}_{m}\left(\omega, y_{m-1}\right)=\{\omega \in \Omega: p(\omega)>0 \text { for some } p \in \mathcal{P}\}
$$

for every $t=1,2, \ldots$. Hence, from the information aggregation assumption, for the selections made before and for $v$ to be the initial announcement and observing that $\mathcal{P} \in \mathcal{A}_{s, v, \mathcal{O}}$, we have that

$$
y_{t}=v=d_{\mathcal{P}}\left(\Pi_{t} \bmod n(\omega) \cap \mathcal{F}_{t-1}(\omega, v), v\right)=d_{\mathcal{P}}\left(\Pi_{t} \bmod n(\omega), v\right) \longrightarrow X(\omega)
$$

for every $\omega \in \Omega$, with $p(\omega)>0$ for some $p \in \mathcal{P}$. ${ }^{36}$ But as we observed, we have that for every $t=1,2, \ldots$ the prediction $y_{t}$ is the same for all $\omega \in \Omega$, with $p(\omega)>0$ for some $p \in \mathcal{P}$. Therefore, from the uniqueness of the limit, $X(\omega)=k$ for every $\omega \in \Omega$ (for some real number $k$ ), with $p(\omega)>0$ for some $p \in \mathcal{P}$. Hence the security is separable under ambiguity.

\footnotetext{
${ }^{36}$ Information aggregation is (by definition) allowed not to hold only for a set of states that is of zero probability for all $p \in \mathcal{P}$. This implies that information should be aggregated at least for those states for which there exists a prior which gives the state strictly positive probability.
} 


\section{Appendix B}

In this part we give a number of examples in order to illustrate the robustness of issues that are encountered when a MSR market is populated by ambiguity averse traders and therefore the extent to which our results are useful.

In the first example we will illustrate how the MSR model can be interpreted as an inventory based market maker. In addition, we will show that in the inventory based interpretation information does not get aggregated always in the presence of ambiguity averse traders. The example is interesting because the interface of real markets might not be a sequential market as MSR, but rather designed with an interface of selling and buying securities (inventory based MM e.g. Inkling Markets) and therefore it is crucial to see if these markets do not aggregate information for some separable under SEU securities when the traders are ambiguity averse.

The second example illustrates a particular case of a separable under SEU security which does not aggregate information when the announcement is in the middle of the value range and for a particular set of priors. The novelty here is that for every prior, belonging in set of priors, its support is the whole state space.

\section{Example 1:}

Consider the state space $\Omega=\left\{\omega_{1}, \omega_{2}, \omega_{3}, \omega_{4}\right\}$, the price function to be the $q(z)=e^{-z}$ where $z$ is the market maker's net inventory. The security is given by $X\left(\omega_{1}\right)=2$, $X\left(\omega_{2}\right)=X\left(\omega_{3}\right)=X\left(\omega_{4}\right)=1$ and the information structure is $\Pi_{1}=\left\{\left\{\omega_{1}, \omega_{2}\right\},\left\{\omega_{3}, \omega_{4}\right\}\right\}$ and $\Pi_{2}=\left\{\left\{\omega_{1}, \omega_{3}\right\},\left\{\omega_{2}, \omega_{4}\right\}\right\}$. The set of priors is the $\mathcal{P}=\operatorname{conv}\left\{\left(0, \frac{1}{3}, \frac{1}{3}, \frac{1}{3}\right),\left(\frac{1}{4}, \frac{1}{4}, \frac{1}{4}, \frac{1}{4}\right)\right\}$. Consider that initially the market maker holds zero inventory of the security (i.e. $z=0$ ).

Firstly, trader 1 makes a myopic decision about how much shares of the security to buy or sell. We assume, for consistency, that the amount of shares belong to $Z=p^{-1}(Y)$, which is compact. Thus it is implied that (for the true state to be either $\omega_{1}$ or $\omega_{2}$ ) trader solves:

$$
\operatorname{maxmin}_{z \in Z} E_{p \in \mathcal{P}}\left[\int_{0}^{z} q(\bar{z})-X(\omega) d \bar{z}\right]=\operatorname{minmax}_{p \in \mathcal{P}} E_{p \in Z}\left[\int_{0}^{z} q(\bar{z})-X(\omega) d \bar{z}\right]
$$


We have the equality by applying the same argument as in the proof of Lemma $2.3 .{ }^{37}$

As in Ostrovsky (2012), given the price function we can define the strictly proper scoring rule $s(X(\omega), y)=\int_{0}^{q^{-1}(y)} q(z)-X(\omega) d z$. We have that the price function $p$ is $1-1$ continuous with continuous inverse function. Therefore we can conclude that in the MSR market, based on that strictly proper scoring rule, the trader solves:

$$
\operatorname{maxmin}_{y \in Y} E_{p \in \mathcal{P}}\left[\int_{0}^{q^{-1}(y)} q(\bar{z})-X(\omega) d \bar{z}\right]=\operatorname{minmax}_{p \in \mathcal{P}} E_{y \in Y}\left[\int_{0}^{q^{-1}(y)} q(\bar{z})-X(\omega) d \bar{z}\right]
$$

38

We shall show that if $z^{*}$ solves the first optimisation problem and $y^{*}$ the second one, then it is $p\left(z^{*}\right)=y^{*}$ and that the revenue or losses are the same:

$$
\operatorname{maxmin}_{z \in Z} E_{p}\left[\int_{0}^{z} q(\bar{z})-X(\omega) d \bar{z}\right]=\operatorname{maxmin}_{y \in Y} E_{p \in \mathcal{P}}\left[\int_{0}^{q^{-1}(y)} q(\bar{z})-X(\omega) d \bar{z}\right]
$$

The conclusion is that the purchase of the optimal amount of shares and the announcement of the myopic prediction are related with a one to one relation using the pricing function and that the two markets are equivalent in terms of revenues and losses.

We can observe that for every $p \in \mathcal{P}$ the amount $z_{p}^{\prime}$ that solves the $\max _{z \in Z} E_{p}\left[\int_{0}^{z} q(\bar{z})-\right.$ $X(\omega) d \bar{z}]$ is unique and such that $p\left(z_{p}^{\prime}\right)=E_{p}[X]$. Similarly, for every $p \in \mathcal{P}$ the prediction $y_{p}^{\prime}$ that solves the $\max _{y \in Y} E_{p}\left[\int_{0}^{q^{-1}(y)} q(\bar{z})-X(\omega) d \bar{z}\right]$ is the $y_{p}^{\prime}=E_{p}[X]$, hence $q^{-1}\left(y_{p}^{\prime}\right)=z_{p}^{\prime}$.

Therefore, for every $p \in \mathcal{P}$ we have that:

$$
E_{p}\left[\int_{0}^{z_{p}^{\prime}} q(\bar{z})-X(\omega) d \bar{z}\right]=E_{p}\left[\int_{0}^{q^{-1}\left(y_{p}^{\prime}\right)} q(\bar{z})-X(\omega) d \bar{z}\right]
$$

We can conclude that:

$$
\min _{p \in \mathcal{P}} E_{p}\left[\int_{0}^{z_{p}^{\prime}} q(\bar{z})-X(\omega) d \bar{z}\right]=\min _{p \in \mathcal{P}} E_{p}\left[\int_{0}^{q^{-1}\left(y_{p}^{\prime}\right)} q(\bar{z})-X(\omega) d \bar{z}\right]
$$

and it is achieved in the same $p^{*}$.

\footnotetext{
${ }^{37}$ We use that $F(z)=\int_{0}^{z} q(\bar{z})-X(\omega) d \bar{z}$ is continuous and we follow the arguments of Lemma 2.3.

${ }^{38}$ Similarly, we follow the arguments of Lemma 2.3 with the continuous function $F(y)=\int_{0}^{q^{-1}(y)} q(\bar{z})-$ $X(\omega) d \bar{z}$.
} 
We conclude that the optimal quantity of shares $z^{*}$ for the ambiguity averse trader is such that $q\left(z^{*}\right)=E_{p^{*}}[X]$ and the optimal prediction $y^{*}$ is such that $y^{*}=E_{p^{*}}[X]$ and thus we get the conclusion. ${ }^{39}$

Finally, the first trader finds the belief that achieves the minimum gives at state $\omega_{1}$ zero probability. From the previous paragraph we conclude that the optimal amount to purchase, $z^{*}$, is such that $p\left(z^{*}\right)=0 * 2+1 * 1=1$ or equivalently (as long as $\mathrm{p}$ is $1-1$ ) $z^{*}=0$. Hence she neither buy or sell any shares (equivalently she would have announced 1 as her prediction, i.e. the price). It is easy to see that the same would happen for every state in the partition $\left\{\omega_{3}, \omega_{4}\right\}$ and for the trader 2 for symmetry reasons. The conclusion is that both traders does not purchase shares from the market maker and no one can infer the true state, even if that would be the case if they pooled their information.

\section{Example 2}

Consider the state space $\Omega=\left\{\omega_{1}, \ldots, \omega_{6}\right\}$ and the partition structure $\Pi_{1}=\left\{\left\{\omega_{1}, \omega_{3}\right\},\left\{\omega_{2}, \omega_{4}\right\}\right.$, $\left.\left\{\omega_{5}, \omega_{6}\right\}\right\}, \Pi_{2}=\left\{\left\{\omega_{1}, \omega_{2}, \omega_{6}\right\},\left\{\omega_{3}, \omega_{4}, \omega_{5}\right\}\right\}, \Pi_{3}=\left\{\left\{\omega_{1}, \omega_{2}\right\},\left\{\omega_{3}, \omega_{5}\right\},\left\{\omega_{4}, \omega_{6}\right\}\right\}$ and the security $X\left(\omega_{1}\right)=X\left(\omega_{5}\right)=0, X\left(\omega_{2}\right)=X\left(\omega_{6}\right)=2, X\left(\omega_{3}\right)=1$ and $X\left(\omega_{4}\right)=-1$.

We will prove, firstly, that this security is separable under SEU.

\section{Claim}

For every $v \in \mathbb{R}$ and every prior $p$ over the state space, if for every $i=1, \ldots, n$ and every $\omega$, with $p(\omega)>0$, it is $E_{p}\left[X \mid \Pi_{i}(\omega)\right]=v$ then for every $\omega$, with $p(\omega)>0$, it is $X(\omega)=v$.

Proof. Let $v \in \mathbb{R}$ and $p$ a prior over the state space $\Omega$.

Case 1: There exists $\omega \in \Omega$ and $i \in\{1, \ldots, n\}$ such that $p\left(\Pi_{i}(\omega)\right)=0$. For every $i \in\{1, \ldots, n\}$ define $A_{i}$ to the the maximal set of $i$ 's information cells such that $p(\pi)>0$ for every $\pi \in A_{i}$.

If the prior is such that, for every $i \in\{1, \ldots, n\}$ it is $E_{p}\left[X \mid \Pi_{i}(\omega)\right]=v$ for every $\omega \in \Omega$, with $p(\omega)>0$, equivalently the prior is such that for every $i \in\{1, \ldots, n\}$ it is $\sum_{\omega^{\prime} \in \Pi_{i}(\omega)} X\left(\omega^{\prime}\right) p\left(\omega^{\prime}\right)=v \cdot p\left(\Pi_{i}(\omega)\right)$ for every $\omega \in \Omega$, with $p(\omega)>0$. Name this set of equations $(*)$.

Then we can conclude that the prior is such that for every $i \in\{1, \ldots, n\}$ it is $\sum_{\omega^{\prime} \in \Pi_{i}(\omega)} X\left(\omega^{\prime}\right) p\left(\omega^{\prime}\right)=$ $v \cdot p\left(\Pi_{i}(\omega)\right)$ for every $\omega \in \Omega$, name this set of equations $(* *)$. This is true because if

\footnotetext{
${ }^{39} \mathrm{By}$ using the saddle point inequality and the uniqueness of the optimal quantity and prediction (given the belief $p^{*}$ ).
} 
there exists $\omega$ such that $\Pi_{i}(\omega) \notin A_{i}$ then $p\left(\Pi_{i}(\omega)\right)=0$ therefore $p\left(\omega^{\prime}\right)=0$ for every $\omega^{\prime} \in \Pi_{i}(\omega)$ and hence $\sum_{\omega^{\prime} \in \Pi_{i}(\omega)} X\left(\omega^{\prime}\right) p\left(\omega^{\prime}\right)=v \cdot p\left(\Pi_{i}(\omega)\right)=0$.

Therefore, if $p$ solves $(*)$ then should solve $(* *)$, too. We will prove that $(* *)$ has solutions that all of them satisfy the claim.

Analytically, the equations $(* *)$, denoting $p=\left(p_{1}, \ldots, p_{6}\right)$, are the followings:

$$
\begin{gathered}
2 p_{2}+2 p_{6}=v p_{1}+v p_{2}+v p_{6} \\
p_{3}-p_{4}=v p_{3}+v p_{4}+v p_{5} \\
p_{3}=v p_{1}+v p_{3} \\
2 p_{2}-p_{4}=v p_{2}+v p_{4} \\
2 p_{6}=v p_{5}+v p_{6} \\
2 p_{2}=v p_{1}+v p_{2} \\
p_{3}=v p_{3}+v p_{5} \\
2 p_{6}-p_{4}=v p_{4}+v p_{6}
\end{gathered}
$$

If $v \neq 0,1,-1,2$ then the system of equations has a unique solution the $p=(0, \ldots, 0)$. Because we want $p$ to be a prior we conclude that there is not such solution in our setting.

If $v=0$ then the solutions are infinite and of the form $p=(a, 0,0,0, b, 0)$ with $a+b=1$. We have that $X\left(\omega_{1}\right)=X\left(\omega_{5}\right)=0$ and hence the definition of separability under SEU is satisfied.

If $v=1$ then the system has $\mathrm{s}$ unique solution the $p=(0,0,1,0,0,0)$. Again the definition of separability under SEU is satisfied for this prior (because $X\left(\omega_{3}\right)=1$ ).

If $v=-1$ then the system has a unique solution the $p=(0,0,0,1,0,0)$. Again the definition of separability under SEU is satisfied for this prior (because $X\left(\omega_{4}\right)=-1$ ).

If $v=2$ then the system has infinite solutions of the form $p=(0, a, 0,0,0, b)$ with $a+b=1$. Again the definition of separability under SEU is satisfied for this prior (because $\left.X\left(\omega_{2}\right)=X\left(\omega_{6}\right)=2\right)$.

Case 2: For every $\omega \in \Omega$ and for every $i \in\{1, \ldots, n\}$ it is $p\left(\Pi_{i}(\omega)\right)>0$. We can proceed exactly as before, concluding that there is not a prior satisfying Case 2 and the hypothesis of the claim. 
Hence we conclude that the security is separable under SEU.

On the other hand, the security is not separable under ambiguity.

To see that, let's take the MM initial announcement to be, say, $y_{0}=0.5$ (which is the middle of the price range) and any strictly proper scoring rule, say the quadratic, and, given the $y_{0}$, any compact and convex set of priors that includes the priors $p=$ $\left(\frac{1}{8}, \frac{1}{8}, \frac{1}{8}, \frac{1}{8}, \frac{3}{8}, \frac{1}{8}\right), p^{\prime}=\left(\frac{6}{18}, \frac{1}{18}, \frac{7}{18}, \frac{2}{18}, \frac{1}{18}, \frac{1}{18}\right)$ and $p^{\prime \prime}=\left(\frac{3}{8}, \frac{1}{8}, \frac{1}{8}, \frac{1}{8}, \frac{1}{8}, \frac{1}{8}\right)$. Then we can see that both condition (ii) and condition (i) of the Definition 2.6 is satisfied, hence the security is not separable under ambiguity (which implies that there is no state in which information gets aggregated). 



\section{Appendix C}

Before we proceed to the main proof, we state a result that will be helpful in order to prove the Theorem 3.6.

Lemma C.1. Consider a convex and compact set of priors $\mathcal{P} \subseteq \Delta(\Omega)$, a player $i \in$ $\{1, \ldots, n\}, \omega^{*}$, for which there exists $p \in \mathcal{P}$ with $p\left(\omega^{*}\right)>0$ and $0<\beta<1$. The function $f: Y^{\mathbb{N}} \times Y^{\mathbb{N}} \longrightarrow \mathbb{R}$ with $f\left(\left(x_{n}\right)_{\mathbb{N}},\left(y_{n}\right)_{\mathbb{N}}\right)=\min _{p \in \mathcal{P}} \sum_{k=0}^{\infty} \beta^{k} E_{p \mid \Pi_{i}\left(\omega^{*}\right)}\left[s\left(x_{k}, X\right)-s\left(y_{k}, X\right)\right]$ is continuous in $\left(\left(x_{n}\right)_{\mathbb{N}},\left(y_{n}\right)_{\mathbb{N}}\right)$.

Proof. As a first step, consider the function $f_{1}: Y^{\mathbb{N}} \times Y^{\mathbb{N}} \longrightarrow Y^{\mathbb{N}} \times Y^{\mathbb{N}}$ with $h\left(\left(x_{n}\right)_{\mathbb{N}},\left(y_{n}\right)_{\mathbb{N}}\right)=$ $\left(\left(s\left(x_{n}, X(\omega)\right)\right)_{\mathbb{N}},\left(s\left(y_{n}, X(\omega)\right)\right)_{\mathbb{N}}\right)$, which is obviously continuous with the product topology for every $\omega \in \Omega .{ }^{40}$

In addition, consider the function $f_{2}: Y^{\mathbb{N}} \times Y^{\mathbb{N}} \longrightarrow \mathbb{R}$ with $f_{2}\left(\left(x_{n}\right)_{\mathbb{N}},\left(y_{n}\right)_{\mathbb{N}}\right)=\sum_{k=0}^{\infty} \beta^{k}\left[x_{k}-\right.$ $\left.y_{k}\right]$. We prove below that this function is continuous in $\left(\left(x_{n}\right)_{\mathbb{N}},\left(y_{n}\right)_{\mathbb{N}}\right)$. Given that, the function $f_{2} \circ f_{1}$ is, then, continuous for every $\omega \in \Omega$.

In order to prove the continuity we use sequences. Hence, consider $\left(\left(x_{n}\right)_{\mathbb{N}}\right)^{m} \longrightarrow_{m}\left(x_{n}\right)_{\mathbb{N}}$ and $\left(\left(y_{n}\right)_{\mathbb{N}}\right)^{m} \longrightarrow_{m}\left(y_{n}\right)_{\mathbb{N}}$. Then we can observe the following:

$$
\begin{gathered}
\left|\sum_{k=0}^{\infty} \beta^{k}\left(x_{k}^{m}-y_{k}^{m}\right)-\sum_{k=0}^{\infty} \beta^{k}\left(x_{k}-y_{k}\right)\right|= \\
\left|\sum_{k=0}^{\infty} \beta^{k}\left(\left(x_{k}^{m}-x_{k}\right)+\left(y_{k}-y_{k}^{m}\right)\right)\right| \leq \\
\sum_{k=0}^{\infty} \beta^{k}\left[\left|x_{k}^{m}-x_{k}\right|+\left|y_{k}-y_{k}^{m}\right|\right]= \\
\sum_{k=0}^{\infty} \beta^{k}\left|x_{k}^{m}-x_{k}\right|+\sum_{k=0}^{\infty} \beta^{k}\left|y_{k}-y_{k}^{m}\right|=
\end{gathered}
$$

\footnotetext{
${ }^{40}$ We assume the metric $d\left(\left(x_{n}\right)_{\mathbb{N}},\left(y_{n}\right)_{\mathbb{N}}\right)=\sum_{k=0}^{\infty} \beta^{k}\left[d_{k}\left(x_{n}, y_{n}\right)\right]$, where $\beta \in(0,1)$ is the discounting factor and $d_{k}$ is the euclidean distance at each coordinate. The topology implied by this metric is equivalent to the product topology in $Y^{\mathbb{N}}$.
} 


$$
d\left(\left(\left(x_{n}\right)_{\mathbb{N}}\right)^{m},\left(x_{n}\right)_{\mathbb{N}}\right)+d\left(\left(\left(y_{n}\right)_{\mathbb{N}}\right)^{m},\left(y_{n}\right)_{\mathbb{N}}\right) .
$$

Hence we get the continuity.

Next, consider the set $\mathcal{P} \subseteq \Delta(\Omega)$ with the weak* topology and finally the set $Y^{\mathbb{N}} \times$ $Y^{\mathbb{N}} \times \mathcal{P}$ with the product topology. Consider the function $h: Y^{\mathbb{N}} \times Y^{\mathbb{N}} \times \mathcal{P} \longrightarrow \mathbb{R}$, with $h\left(\left(x_{n}\right)_{\mathbb{N}},\left(y_{n}\right)_{\mathbb{N}}, p\right)=\sum_{k=0}^{\infty} \beta^{k} E_{p_{\mid \Pi_{i}\left(\omega^{*}\right)}}\left[s\left(x_{k}, X\right)-s\left(y_{k}, X\right)\right]$. We will prove that this function is continuous in $\left(\left(x_{n}\right)_{\mathbb{N}},\left(y_{n}\right)_{\mathbb{N}}, p\right)$.

Firstly, we can observe that

$$
\begin{gathered}
h\left(\left(x_{n}\right)_{\mathbb{N}},\left(y_{n}\right)_{\mathbb{N}}, p\right)= \\
\sum_{k=0}^{\infty} \beta^{k} E_{p_{\mid \Pi_{i}\left(\omega^{*}\right)}}\left[s\left(x_{k}, X\right)-s\left(y_{k}, X\right)\right]= \\
E_{p_{\mid \Pi_{i}\left(\omega^{*}\right)}}\left[\sum_{k=0}^{\infty} \beta^{k}\left(s\left(x_{k}, X\right)-s\left(y_{k}, X\right)\right)\right]= \\
\sum_{\omega \in \Pi_{i}\left(\omega^{*}\right)} \frac{p(\omega)}{p\left(\Pi_{i}\left(\omega^{*}\right)\right)}\left[\sum_{k=0}^{\infty} \beta^{k}\left(s\left(x_{k}, X\right)-s\left(y_{k}, X\right)\right)\right]
\end{gathered}
$$

where the first equality is because of the Beppo-Levi theorem.

Take a sequence $\left(\left(\left(x_{n}\right)_{\mathbb{N}}\right)^{j},\left(\left(y_{n}\right)_{\mathbb{N}}\right)^{j}, p_{j}\right)_{j \in \mathbb{N}} \in Y^{\mathbb{N}} \times Y^{\mathbb{N}} \times \mathcal{P}$ such that

$$
\left(\left(\left(x_{n}\right)_{\mathbb{N}}\right)^{j},\left(\left(y_{n}\right)_{\mathbb{N}}\right)^{j}, p_{j}\right) \longrightarrow_{j}\left(\left(x_{n}\right)_{\mathbb{N}},\left(y_{n}\right)_{\mathbb{N}}, p\right) .
$$

Then:

$$
h\left(\left(\left(x_{n}\right)_{\mathbb{N}}\right)^{j},\left(\left(y_{n}\right)_{\mathbb{N}}\right)^{j}, p_{j}\right)=\sum_{\omega \in \Pi_{i}\left(\omega^{*}\right)} p_{j}(\omega)\left[\sum_{k=0}^{\infty} \beta^{k}\left(s\left(x_{k}^{j}, X(\omega)\right)-s\left(y_{k}^{j}, X(\omega)\right)\right)\right] .
$$

For every $\omega \in \Pi_{i}\left(\omega^{*}\right)$ we have that $\sum_{k=0}^{\infty} \beta^{k}\left[s\left(x_{k}, X(\omega)\right)-s\left(y_{k}, X(\omega)\right)\right]$ is continuous in $\left(\left(x_{n}\right)_{\mathbb{N}},\left(y_{n}\right)_{\mathbb{N}}\right)$, as long as it is the function $f_{2} \circ f_{1}$. In addition, $p_{j} \longrightarrow p$ in the weak* topology if and only if $p_{j}(\omega) \longrightarrow_{j} p(\omega)$, for every $\omega \in \Omega$. Therefore, we conclude:

$$
\begin{gathered}
h\left(\left(\left(x_{n}\right)_{\mathbb{N}}\right)^{j},\left(\left(y_{n}\right)_{\mathbb{N}}\right)^{j}, p_{j}\right)= \\
\sum_{\omega \in \Pi_{i}\left(\omega^{*}\right)} \frac{p_{j}(\omega)}{p_{j}\left(\Pi_{i}\left(\omega^{*}\right)\right)}\left[\sum_{k=0}^{\infty} \beta^{k}\left(s\left(x_{k}^{j}, X\right)-s\left(y_{k}^{j}, X\right)\right)\right] \underset{j}{\longrightarrow} \\
\sum_{\omega \in \Pi_{i}\left(\omega^{*}\right)} \frac{p(\omega)}{p\left(\Pi_{i}\left(\omega^{*}\right)\right)}\left[\sum_{k=0}^{\infty} \beta^{k}\left(s\left(x_{k}, X\right)-s\left(y_{k}, X\right)\right)\right]= \\
h\left(\left(x_{n}\right)_{\mathbb{N}},\left(y_{n}\right)_{\mathbb{N}}, p\right) .
\end{gathered}
$$

Hence we can conclude that the function $h$ is continuous in $\left(\left(x_{n}\right)_{\mathbb{N}},\left(y_{n}\right)_{\mathbb{N}}, p\right)$. 
The next step is to apply appropriately the maximum theorem. First observe that we can see $\mathcal{P}$ as a metric space, because $\Omega$ is separable under SEU. In addition, consider the compact valued correspondence $\mathcal{C}: Y^{\mathbb{N}} \times Y^{\mathbb{N}} \longrightarrow \mathcal{P}$ with $\mathcal{C}\left(\left(x_{n}\right)_{\mathbb{N}},\left(y_{n}\right)_{\mathbb{N}}\right)=\mathcal{P}$ for every $\left(\left(x_{n}\right)_{\mathbb{N}},\left(y_{n}\right)_{\mathbb{N}}\right) \in Y^{\mathbb{N}} \times Y^{\mathbb{N}}$, which is both upper and lower hemicontinuous, as it is constant correspondence. Therefore, by the maximum theorem we get that $\min _{p \in \mathcal{P}} h\left(\left(x_{n}\right)_{\mathbb{N}},\left(y_{n}\right)_{\mathbb{N}}, p\right)$ is continuous in $\left(\left(x_{n}\right)_{\mathbb{N}},\left(y_{n}\right)_{\mathbb{N}}\right)$, hence we get the result of the theorem.

Next we proceed to the proof of Theorem 3.6 (i).

Proof. The proof proceeds in three steps. Firstly, we will conclude that on equilibrium path the continuation games for each player $i$, at every realised true state and at every time that she trades (i.e. in every i's self) should converge to some non negative real number. Then, using the fact that the strategies converge and properties of the MSR we conclude that the continuation payoffs should converge to zero. Finally, we conclude that in the limit the revision proof predictions are the myopic ones and then by separability under SEU under ambiguity we can show that information gets aggregated.

\section{Step 1:}

Let assume that there exists player $i$, state $\omega^{*}$, for which there exists $p \in \mathcal{P}$ with $p\left(\omega^{*}\right)>0$, such that $V_{i+n t}\left(H^{i-1+n t}\left(y^{*}\left(\omega^{*}\right)\right),\left(y^{*^{i}}, y^{*^{-i}}\right), \omega^{*}\right)$ converges (in $\left.t\right)$ to some $c_{i}^{\Pi_{i}\left(\omega^{*}\right)}<0$.

Consider the tail of the sequence from $H^{i-1+n t}\left(y^{*}\left(\omega^{*}\right)\right)$ and onwards, such that $t>T$ where $T$ is the round that the public information reaches its finest set. In other words, $H^{i-1+n t}\left(y^{*}(\omega)\right)$ is the on equilibrium path history until $i-1+n t$ prediction, and the public information $\mathcal{F}_{T}^{y^{*}}\left(\omega^{*}\right)$ is the finest set of states that are considered possible to have been realised (with respect to the history).

We observe that:

$$
\begin{gathered}
V_{i+n t}\left(H^{i-1+n t}\left(y^{*}\left(\omega^{*}\right)\right),\left(y^{*^{i}}, y^{*^{-i}}\right), \omega^{*}\right)= \\
\min _{p \in \mathcal{P}_{\mathcal{F}_{T}^{y^{*}}\left(\omega^{*}\right) \cap \Pi_{i}\left(\omega^{*}\right)}} \sum_{k=0}^{\infty} \beta^{n k} E_{p}\left[s\left(y_{i+n k+n t}^{*}\left(\Pi_{i}\left(\omega^{*}\right), H^{i-1+n k+n t}\left(y^{*}\left(\omega^{\prime}\right)\right)\right), X\right)-\right. \\
\left.s\left(y_{i-1+n k+n t}^{*}\left(\Pi_{i-1}\left(\omega^{\prime}\right), H^{i-2+n k+n t}\left(y^{*}\left(\omega^{\prime}\right)\right)\right), X\right)\right]
\end{gathered}
$$

Therefore, because $\mathcal{F}_{T}^{y^{*}}\left(\omega^{*}\right)$ is the finest set of states that are considered possible to have been realised, for $t>T$, for every $i=1, \ldots, n$ and $k \in \mathbb{N}$ we have that

$$
y_{i+n k+n t}^{*}\left(\Pi_{i}\left(\omega^{*}\right), H^{i-1+n k+n t}\left(y^{*}\left(\omega^{*}\right)\right)\right)=y_{i+n k+n t}^{*}\left(\Pi_{i}(\omega), H^{i-1+n k+n t}\left(y^{*}(\omega)\right)\right)
$$


for every $\omega \in \mathcal{F}_{T}^{y^{*}}\left(\omega^{*}\right)$, for which there exists $p \in \mathcal{P}$ with $p(\omega)>0$ (otherwise more information would be revealed).

This in turn implies that for $t>T$, for every $i=1, \ldots, n$ and $k \in \mathbb{N}$ it is

$$
H^{i-1+n k+n t}\left(y^{*}\left(\omega^{*}\right)\right)=H^{i-1+n k+n t}\left(y^{*}(\omega)\right)
$$

for every $\omega \in \mathcal{F}_{T}^{y^{*}}\left(\omega^{*}\right)$, for which there exists $p \in \mathcal{P}$ with $p(\omega)>0$.

Hence, considering the sequences

$$
\left(\left(x_{k}\right)_{k \in \mathbb{N}}\right)^{t}=\left(\left(y_{i+n k+n t}^{*}\left(\Pi_{i}\left(\omega^{*}\right), H^{i-1+n k+n t}\left(y^{*}\left(\omega^{*}\right)\right)\right)\right)_{k \in \mathbb{N}}\right)^{t}
$$

and

$$
\left(\left(y_{k}\right)_{k \in \mathbb{N}}\right)^{t}=\left(\left(y_{i-1+n k+n t}^{*}\left(\Pi_{i-1}\left(\omega^{*}\right), H_{y^{*}}^{i-2+n k+n t}\left(\omega^{*}\right)\right)\right)_{k \in \mathbb{N}}\right)^{t}
$$

we conclude that for every $i=1, \ldots, n$ :

$$
\left(\left(y_{i+n k+n t}^{*}\left(\Pi_{i}\left(\omega^{*}\right), H^{i-1+n k+n t}\left(y^{*}\left(\omega^{*}\right)\right)\right)\right)_{k \in \mathbb{N}}\right)^{t} \underset{t}{\longrightarrow}\left(y_{i, \infty}^{*}\left(\Pi_{i}\left(\omega^{*}\right), H^{\infty}\left(y^{*}\left(\omega^{*}\right)\right)\right)\right)_{k \in \mathbb{N}}
$$

and

$$
\left(\left(y_{i-1+n k+n t}^{*}\left(\Pi_{i-1}\left(\omega^{*}\right), H^{i-2+n k+n t}\left(y^{*}\left(\omega^{*}\right)\right)\right)_{k \in \mathbb{N}}\right)\right)^{t} \underset{t}{\longrightarrow}\left(y_{i-1, \infty}^{*}\left(\Pi_{i-1}\left(\omega^{*}\right), H^{\infty}\left(y^{*}\left(\omega^{*}\right)\right)\right)\right)_{k \in \mathbb{N}}{ }^{41}
$$

This is because the convergence of the sequences is in the product topology and for every $k \in \mathbb{N}$ it is $y_{i+n k+n t}^{*}\left(\Pi_{i}\left(\omega^{*}\right), H^{i-1+n k+n t}\left(y^{*}(\omega)\right)\right) \longrightarrow_{t} y_{i, \infty}^{*}\left(\Pi_{i}\left(\omega^{*}\right), H^{\infty}\left(y^{*}\left(\omega^{*}\right)\right)\right)$ for every $i$ (by the converging assumption).

Using Lemma C.1 we conclude that the limit of the tail exists and it is:

$\min _{p \in \mathcal{P}_{\mathcal{F}_{T}^{y^{*}}\left(\omega^{*}\right) \cap \Pi_{i}\left(\omega^{*}\right)}} \frac{E_{p}\left[s\left(y_{i, \infty}^{*}\left(\Pi_{i}\left(\omega^{*}\right), H^{\infty}\left(y^{*}\left(\omega^{\prime}\right)\right)\right), X\right)-s\left(y_{i-1, \infty}^{*}\left(\Pi_{i-1}\left(\omega^{\prime}\right), H^{\infty}\left(y^{*}\left(\omega^{\prime}\right)\right)\right), X\right)\right]}{1-\beta^{n}}$.

Name the limit $c^{\Pi_{i}\left(\omega^{*}\right)}$. Then finally it should be the case that there exists $t_{0} \in \mathbb{N}$ such that for every $t \geq t_{0}>>T$ it is

$$
\begin{gathered}
V_{i+n t}\left(H^{i-1+n t}\left(y^{*}\left(\omega^{*}\right)\right),\left(y^{*^{i}}, y^{*^{-i}}\right), \omega^{*}\right)= \\
\min _{p \in \mathcal{P}_{\mathcal{F}_{T}^{*}}\left(\omega^{*}\right) \cap \Pi_{i}\left(\omega^{*}\right)} \sum_{k=t}^{\infty} \beta^{n k-n t} E_{p}\left[s\left(y_{i+n k}^{*}\left(\Pi_{i}\left(\omega^{*}\right), H^{i-1+n k}\left(y^{*}\left(\omega^{\prime}\right)\right)\right), X\right)-\right.
\end{gathered}
$$

\footnotetext{
${ }^{41}$ Because we are after the time the finest information for every player is achieved the states $\omega \in$ $\Pi_{i}\left(\omega^{*}\right) \cap \mathcal{F}_{T}^{y^{*}}(\omega)$ so not reveal information about as the strategic predictions over them are constant. Hence, we select to represent that constant value by using the realised state $\omega^{*}$. However, when need to have an expectation we turn to the normal notation.
} 


$$
\left.s\left(y_{i-1+n k}^{*}\left(\Pi_{i-1}\left(\omega^{\prime}\right), H^{i-2+n k}\left(y^{*}\left(\omega^{\prime}\right)\right)\right), X\right)\right]<0 .^{42}
$$

Consider round $t_{0}$, the equilibrium history $H^{i+n t_{0}-1}\left(y^{*}\left(\omega^{*}\right)\right)$ and the implied public information $\mathcal{F}_{i+n t_{0}-1}^{y^{*}}\left(\omega^{*}\right)$. The set of states that the player thinks possible to have been realised is $\Pi_{i}\left(\omega^{*}\right) \cap \mathcal{F}_{i+n t_{0}-1}^{y^{*}}\left(\omega^{*}\right){ }^{43}$ According to the assumption made in this step, if player $i$ follows his equilibrium strategy then the continuation payoff is negative. However, we can define the following deviation strategy, assuming that $-i$ follow their equilibrium strategies:

(i) Define $y_{i+n t_{0}}^{\prime}\left(\Pi_{i}\left(\omega^{*}\right), H^{i+n t_{0}-1}\left(y^{*}\left(\omega^{*}\right)\right)\right)$ to be whatever the previous player predicted. The set $\Pi_{i}\left(\omega^{*}\right) \cap \mathcal{F}_{i+n t_{0}-1}^{y^{*}}\left(\omega^{*}\right)$ can be partitioned in the following way:

For $\omega \in \Pi_{i}\left(\omega^{*}\right) \cap \mathcal{F}_{i+n t_{0}-1}^{y^{*}}\left(\omega^{*}\right)$, with $p(\omega)>0$ for some $p \in \mathcal{P}$, the equilibrium play of $-i$ after the deviation $y_{i+n t_{0}}^{\prime}\left(\Pi_{i+n t_{0}}, H^{i+n t_{0}-1}\left(y^{*}\left(\omega^{*}\right)\right)\right)$, let's denote that path with $H_{\omega}$, leads to $i$ 's information set

$$
\left\{H_{y^{*}}^{i+n t_{0}-1}\left(\omega^{*}\right) \cup\left(y_{i+n t_{0}}^{\prime}\left(\Pi_{i}\left(\omega^{*}\right), H^{i+n t_{0}-1}\left(y^{*}\left(\omega^{*}\right)\right)\right) \cup H_{\omega}, \omega^{\prime}\right): \omega^{\prime} \in \Pi_{i}(\omega)=\Pi_{i}\left(\omega^{*}\right)\right\} .
$$

According to Definition 3.3 (iii), the states that are considered to have been realised in this information set are the ones consistent with the path and the beliefs are derived by prior by prior updating. Consider as one member of the partition that set, and apply the same reasoning for every $\omega \in \Pi_{i}\left(\omega^{*}\right) \cap \mathcal{F}_{i+n t_{0}-1}^{y^{*}}\left(\omega^{*}\right)$, with $p(\omega)>0$ for some $p \in \mathcal{P}$. To sum up, we conclude a partition of

$$
\Pi_{i}\left(\omega^{*}\right) \cap \mathcal{F}_{i+n t_{0}-1}^{*}\left(\omega^{*}\right)=\bigcup_{j=1}^{m}\left(\Pi_{i}\left(\omega^{*}\right) \cap \mathcal{F}_{i+n t_{0}+n-1}^{y^{\prime}, j}\right)
$$

and let's denote $H_{y^{\prime}}^{1}, \ldots, H_{y^{\prime}}^{m}$ the path of $-i$ that leads to $\Pi_{i}\left(\omega^{*}\right) \cap \mathcal{F}_{i+n t_{0}+n-1}^{y^{\prime}, 1}, \ldots, \Pi_{i}\left(\omega^{*}\right) \cap$ $\mathcal{F}_{i+n t_{0}+n-1}^{y^{\prime}, m}$ respectively. ${ }^{44}$

(ii) In each of those sets, let assume without loss in generality that it is after the $H_{m}^{j}$ for some $1 \leq j \leq n$, we define the deviation strategy as follows:

(a) If $V_{i+n t_{0}+n}\left(H^{i+n t_{0}-1}\left(y^{*}\left(\omega^{*}\right)\right) \cup\left\{y_{i+n t_{0}}^{\prime}\left(\Pi_{i}\left(\omega^{*}\right), H^{i+n t_{0}-1}\left(y^{*}\left(\omega^{*}\right)\right)\right)\right\} \cup H_{m}^{j},\left(y^{*^{i}}, y^{*^{-i}}\right), \omega^{*}\right)$ is non negative, then the deviation strategy coincides with the equilibrium at every succeeding information set (including the one at round $t_{0}+1$ ).

(b) If not, then define the deviation action

$y_{i+n t_{0}+n}^{\prime}\left(\Pi_{i}\left(\omega^{*}\right), H^{i+n t_{0}-1}\left(y^{*}\left(\omega^{*}\right)\right) \cup\left\{y_{i+n t_{0}}^{\prime}\left(\Pi_{i}\left(\omega^{*}\right), H^{i+n t_{0}-1}\left(y^{*}\left(\omega^{*}\right)\right)\right)\right\} \cup H_{m}^{j}\right)$

to be the same with what the previous player predicted.

\footnotetext{
${ }^{42}$ Notice here that we can interchange the sum with the expectation using Beppo-Levi theorem.

${ }^{43}$ Although it is $\mathcal{F}_{i+n t_{0}-1}^{y^{*}}\left(\omega^{*}\right)=\mathcal{F}_{T}^{y^{*}}\left(\omega^{*}\right)$, we use the more analytic notation in order to make clear the information set after the deviation, in which case it is not always true in general that no more information communication occurs.

${ }^{44}$ Of course it can be the case that the partition is trivial, in the sense that it contains the initial set as its only element. We assumed a more general occasion in order to have the arguments in their more general form.
} 
If (b) happens, then for the corresponding information set and the history until it is reached, we repeat the same reasoning as in $(i)$ and $(i i)$.

(iii) We define the deviation strategy to be the same with he equilibrium strategy everywhere else that is not defined by the previous procedure.

Having defined the deviation strategy, we can observe now that estimating the utility of the deviation strategy at time $i+n t_{0}$, when the history until that point is the equilibrium path and $-i$ follow their equilibrium strategies, we have that it is:

$$
\begin{aligned}
& V_{i+n t_{0}}\left(H^{i+n t_{0}-1}\left(y^{*}\left(\omega^{*}\right)\right),\left(y^{\prime i}, y^{*^{-i}}\right), \omega^{*}\right)= \\
& \min _{p \in \mathcal{P}_{\mathcal{F}_{i+n t_{0}-1}^{y^{*}}\left(\omega^{*}\right) \cap \Pi_{i}\left(\omega^{*}\right)}} \sum_{k=t_{0}+1}^{\infty} \beta^{n k-n t_{0}} E_{p}\left[s \left(y _ { i + n k } ^ { \prime } \left(\Pi_{i}\left(\omega^{*}\right), H^{i-1+n t_{0}}\left(y^{*}\left(\omega^{*}\right)\right) \cup\right.\right.\right. \\
& \left.\left.\left\{y_{i+n t_{0}}^{\prime}\left(\Pi_{i}\left(\omega^{*}\right), H^{i-1+n t_{0}}\left(y^{*}\left(\omega^{*}\right)\right)\right)\right\} \cup H_{i+n t_{0}}^{i-1+n k}\left(\left(y^{\prime i}, y^{*^{-i}}\right)\left(\omega^{\prime}\right)\right)\right), X\right)-s\left(y _ { i - 1 + n k } ^ { * } \left(\Pi_{i-1}\left(\omega^{\prime}\right), H^{i-1+n t_{0}}\left(y^{*}\left(\omega^{*}\right)\right) \cup\right.\right. \\
& \left.\left.\left.\left\{y_{i+n t_{0}}^{\prime}\left(\Pi_{i}\left(\omega^{*}\right), H^{i-1+n t_{0}}\left(y^{*}\left(\omega^{*}\right)\right)\right)\right\} \cup H_{i+n t_{0}}^{i-2+n k}\left(\left(y^{\prime i}, y^{*^{-i}}\right)\left(\omega^{\prime}\right)\right)\right), X\right)\right] \text {. }
\end{aligned}
$$

For $t \geq t_{0}$ define:

$f_{t}(\omega)=\left[\sum_{k=t}^{\infty} \beta^{n k-n t_{0}}\left(s\left(y_{i+n k}^{\prime}\left(\Pi_{i}(\omega), H^{i+n t_{0}-1}\left(y^{*}\left(\omega^{*}\right)\right) \cup H_{i+n t_{0}-1}^{i+n k-1}\left(\left(y^{\prime i}, y^{*^{-i}}\right)(\omega)\right)\right), X\right)-\right.\right.$ $\left.\left.s\left(y_{i-1+n k}^{*}\left(\Pi_{i-1}(\omega), H^{i-2+n t}\left(y^{*}\left(\omega^{*}\right)\right) \cup H_{i+n t_{0}-2}^{i+n k-2}\left(\left(y^{\prime i}, y^{*^{-i}}\right)(\omega)\right)\right), X\right)\right)\right]$, therefore we have that:

$$
\begin{gathered}
V_{i+n t_{0}}\left(H^{i+n t_{0}-1}\left(y^{*}\left(\omega^{*}\right)\right),\left(y^{\prime i}, y^{*^{-i}}\right), \omega^{*}\right)= \\
\min _{p \in \mathcal{P}_{\mathcal{F}_{i+n t_{0}-1}^{y^{*}}\left(\omega^{*}\right) \cap \Pi_{i}\left(\omega^{*}\right)}} E_{p}\left[f_{t_{0}}\right] .
\end{gathered}
$$

Define the set:

$A^{c}=\left\{\omega \in \mathcal{F}_{i+n t_{0}-1}^{y^{*}}\left(\omega^{*}\right) \cap \Pi_{i}\left(\omega^{*}\right): V_{i+n t_{0}+n k}\left(H^{i+n t_{0}-1}\left(y^{*}\left(\omega^{*}\right)\right) \cup H_{i+n t_{0}-1}^{i+n t_{0}+k n-1}\left(\left(y^{\prime i}, y^{*-i}\right)(\omega)\right),\left(y^{*^{i}}, y^{*^{-i}}\right), \omega^{*}\right)<\right.$ 0 for every $k \geq 0\}$. Then for every $\omega \in A^{c}$ we have $f_{t_{0}}(\omega)=0$, by definition of $y^{\prime i}$.

For every $\omega \in A$ define:

$k_{\omega}=\min \left\{k: V_{i+n t_{0}+n k}\left(H^{i+n t_{0}-1}\left(y^{*}\left(\omega^{*}\right)\right) \cup H_{i+n t_{0}-1}^{i+n t_{0}+k n-1}\left(\left(y^{\prime i}, y^{*-i}\right)(\omega)\right),\left(y^{*^{i}}, y^{*^{-i}}\right), \omega^{*}\right) \geq 0\right\}$.

We know that the minimum is well defined by the definition of $A$.

By the definition of $y^{\prime i}$ we have that for every $\omega \in \mathcal{F}_{T}^{y^{*}}\left(\omega^{*}\right) \cap \Pi_{i}\left(\omega^{*}\right)$ :

$$
V_{i+n t_{0}+n k_{\omega}}\left(H^{i+n t_{0}-1}\left(y^{*}\left(\omega^{*}\right)\right) \cup H_{i+n t_{0}-1}^{i+n t_{\omega} n-1}\left(\left(y^{\prime i}, y^{*-i}\right)(\omega)\right),\left(y^{\prime i}, y^{*^{-i}}\right), \omega^{*}\right)=
$$




$$
V_{i+n t_{0}+n k_{\omega}}\left(H^{i+n t_{0}-1}\left(y^{*}\left(\omega^{*}\right)\right) \cup H_{i+n t_{0}-1}^{i+n t_{0}+k_{\omega} n-1}\left(\left(y^{\prime i}, y^{*-i}\right)(\omega)\right),\left(y^{*^{i}}, y^{*^{-i}}\right), \omega^{*}\right) \geq 0
$$

and for the preceding information sets at rounds $t_{0}, \ldots, t_{0}+k_{\omega}$ the deviation action of $i$ is playing whatever the previous.

For every $\omega \in A$ set

$A_{\omega}=\left\{\omega^{\prime} \in A: H^{i+n t_{0}-1}\left(y^{*}\left(\omega^{*}\right)\right) \cup H_{i-1+n t_{0}}^{i-1+n k_{\omega}}\left(\left(y^{\prime i}, y^{*-i}\right)\left(\omega^{\prime}\right)\right)=H^{i+n t_{0}-1}\left(y^{*}\left(\omega^{*}\right)\right) \cup\right.$ $\left.H_{i-1+n t_{0}}^{i-1+n t_{\omega}}\left(\left(y^{\prime i}, y^{*-i}\right)(\omega)\right)\right\}$.

Intuitively, this is the set of states that $i$ thinks are possible to happen, when the realised state is $\omega^{*}$, the round is $\left(t_{0}+k_{\omega}\right)$ and the history is $H^{i+n t_{0}-1}\left(y^{*}\left(\omega^{*}\right)\right) \cup H_{i-1+n t_{0}+n k_{\omega}}^{i-1+n t_{0}}\left(\left(y^{\prime i}, y^{*-i}\right)(\omega)\right)$. In fact, these sets form a partition of the set A.Here we use Definition 3.3 (iii). Name the partition $A_{\omega_{1}}, \ldots, A_{\omega_{m}}$. Essentially, it is the joint information between the public information created by the corresponding history and $i$ 's private signal.

Next, we can observe that:

$$
\begin{gathered}
V_{i+n t_{0}}\left(H^{i+n t_{0}-1}\left(y^{*}\left(\omega^{*}\right)\right),\left(y^{\prime i}, y^{*-i}\right), \omega^{*}\right)= \\
E_{p^{*}}\left[f_{t_{0}}\right]= \\
\sum_{\omega \in A} f_{t_{0}}(\omega) p^{*}(\omega)
\end{gathered}
$$

for some $p^{*} \in \mathcal{P}_{\mathcal{F}_{i+n t_{0}-1}^{y^{*}}\left(\omega^{*}\right) \cap \Pi_{i}\left(\omega^{*}\right)^{*}}$.

But

$$
\begin{gathered}
\sum_{\omega \in A} f_{t_{0}}(\omega) p^{*}(\omega)= \\
p^{*}\left(A_{\omega_{1}}\right) \sum_{\omega \in A_{\omega_{1}}} \beta^{n k_{\omega_{1}}} f_{t_{0}+k \omega_{1}}(\omega) \frac{p^{*}(\omega)}{p^{*}\left(A_{\omega_{1}}\right)}+\ldots+p^{*}\left(A_{\omega_{m}}\right) \sum_{\omega \in A_{\omega_{m}}} \beta^{n k_{\omega_{m}}} f_{t_{0}+k \omega_{m}}(\omega) \frac{p^{*}(\omega)}{p^{*}\left(A_{\omega_{m}}\right)} \cdot{ }^{45}
\end{gathered}
$$

In addition, for every $\omega_{1}, \ldots, \omega_{m}$ it holds that:

$$
\begin{aligned}
& \sum_{\omega \in A_{\omega_{j}}} \beta^{n k_{\omega_{j}}} f_{t_{0}+k \omega_{j}}(\omega) \frac{p^{*}(\omega)}{p^{*}\left(A_{\omega_{j}}\right)} \geq \\
& \beta^{n k_{\omega_{j}}} V_{i+n t_{0}+n k_{\omega_{j}}}\left(H^{i+n t_{0}-1}\left(y^{*}\left(\omega^{*}\right)\right) \cup H_{i+n t_{0}-1}^{i+n t_{0}+k_{\omega_{j}} n-1}\left(\left(y^{\prime i}, y^{*-i}\right)\left(\omega_{j}\right)\right),\left(y^{\prime i}, y^{*^{-i}}\right), \omega^{*}\right)= \\
& \beta^{n k_{\omega_{j}}} V_{i+n t_{0}+n k_{\omega_{j}}}\left(H^{i+n t_{0}-1}\left(y^{*}\left(\omega^{*}\right)\right) \cup H_{i+n t_{0}-1}^{i+n t_{0}+k_{\omega} n-1}\left(\left(y^{\prime i}, y^{*-i}\right)\left(\omega_{j}\right)\right),\left(y^{*^{i}}, y^{*^{-i}}\right), \omega^{*}\right) \geq 0 .
\end{aligned}
$$

Hence

$$
V_{i+n t_{0}}\left(H^{i+n t_{0}-1}\left(y^{*}\left(\omega^{*}\right)\right),\left(y^{i}, y^{*-i}\right), \omega^{*}\right) \geq 0 .^{46}
$$

Assume now that we are on deviation strategy's path at round, say $t_{0}+\lambda$. If for every $\omega \in \mathcal{F}_{i+n\left(t_{0}+\lambda\right)-1}^{y^{*}}\left(\omega^{*}\right) \cap \Pi_{i}\left(\omega^{*}\right)$ with $k_{\omega} \leq t_{0}+\lambda$ then the deviation strategy

\footnotetext{
${ }^{45}$ In this argument we use Definition 3.3 (iii): the deviator (i.e. trader $i$ ) updates prior by prior.

${ }^{46}$ In this argument we use Definition 3.3 (iii): the deviator (i.e. trader $i$ ) updates prior by prior.
} 
in the continuation is the same as the equilibrium's one. ${ }^{47}$ Otherwise, we can apply the previous reasoning for the new information set (essentially the only change in the arguments above is that instead of having history $H^{i+n t_{0}-1}\left(y^{*}\left(\omega^{*}\right)\right)$ we would have $H^{i+n t_{0}-1}\left(y^{*}\left(\omega^{*}\right)\right) \cup H_{i+n t_{0}-1}^{i+n t_{0}+(k+\lambda) n-1}\left(\left(y^{\prime i}, y^{*-i}\right)(\omega)\right)$.

In addition, outside the deviation path we have that the payoffs are the same, by its definition, too. Hence, we get a contradiction, because $y^{*}$ is revision proof.

Hence we conclude that $c_{i}^{\Pi_{i}\left(\omega^{*}\right)} \geq 0$, for every $i$ and $\omega^{*}$, for which $p\left(\omega^{*}\right)>0$ for some $p \in \mathcal{P}$.

\section{Step 2:}

Because of Step 1, in the limit we have that for every $\omega^{*}$, such that there exists $p \in \mathcal{P}$ with $p\left(\omega^{*}\right)>0$, it is:

$\min _{p \in \mathcal{P}_{\mathcal{F}_{T}^{y^{*}}\left(\omega^{*}\right) \cap \Pi_{i}\left(\omega^{*}\right)}} \frac{E_{p}\left[s\left(y_{i, \infty}^{*}\left(\Pi_{i}\left(\omega^{*}\right), H^{\infty}\left(y^{*}\left(\omega^{\prime}\right)\right)\right), X\right)-s\left(y_{i-1, \infty}^{*}\left(\Pi_{i-1}\left(\omega^{\prime}\right), H^{\infty}\left(y^{*}\left(\omega^{\prime}\right)\right)\right), X\right)\right]}{1-\beta^{n}} \geq 0$.

Therefore, for every $p \in \mathcal{P}$ it is:

$E_{p \mid \Pi_{i}\left(\omega^{*}\right) \cap \mathcal{F}_{T}^{y^{*}\left(\omega^{*}\right)}}\left[s\left(y_{i, \infty}^{*}\left(\Pi_{i}\left(\omega^{*}\right), H^{\infty}\left(y^{*}\left(\omega^{\prime}\right)\right)\right), X\right)-s\left(y_{i-1, \infty}^{*}\left(\Pi_{i-1}\left(\omega^{\prime}\right), H^{\infty}\left(y^{*}\left(\omega^{\prime}\right)\right)\right), X\right)\right] \geq 0$

and hence it is:

$p\left(\Pi_{i}\left(\omega^{*}\right) \cap \mathcal{F}_{T}^{y^{*}}\left(\omega^{*}\right)\right) E_{p \mid \Pi_{i}\left(\omega^{*}\right) \cap \mathcal{F}_{T}^{y^{*}}\left(\omega^{*}\right)}\left[s\left(y_{i, \infty}^{*}\left(\Pi_{i}\left(\omega^{*}\right), H^{\infty}\left(y^{*}\left(\omega^{\prime}\right)\right)\right), X\right)-s\left(y_{i-1, \infty}^{*}\left(\Pi_{i-1}\left(\omega^{\prime}\right), H^{\infty}\left(y^{*}\left(\omega^{\prime}\right)\right)\right), X\right)\right.$

.48

We have that $\mathcal{F}_{T}^{y^{*}}\left(\omega^{*}\right)=\mathcal{F}_{T}^{y^{*}}(\omega)$ for every $\omega \in \mathcal{F}_{T}^{y^{*}}\left(\omega^{*}\right)$. Summing over $\mathcal{C}_{i}=\left\{\Pi_{i}(\omega)\right.$ : $\left.\omega \in \mathcal{F}_{T}^{y^{*}}\left(\omega^{*}\right)\right\}$ and for every $p \in \mathcal{P}$ it is:

$$
\sum_{\Pi_{i}(\omega) \in \mathcal{C}_{i}} p\left(\Pi_{i}(\omega) \cap \mathcal{F}_{T}^{y^{*}}\left(\omega^{*}\right)\right) E_{p \mid \Pi_{i}(\omega) \cap \mathcal{F}_{T}^{y^{*}}\left(\omega^{*}\right)}\left[s\left(y_{i, \infty}^{*}\left(\Pi_{i}(\omega), H^{\infty}\left(y^{*}\left(\omega^{\prime}\right)\right)\right), X\right)-\right.
$$

\footnotetext{
${ }^{47}$ Notice here that if there exists $\omega \in \mathcal{F}_{i+n\left(t_{0}+\lambda\right)-1}^{y^{*}}\left(\omega^{*}\right) \cap \Pi_{i}\left(\omega^{*}\right)$ it is $k_{\omega} \leq t_{0}+\lambda$ then the same applies for every $\omega \in \mathcal{F}_{i+n\left(t_{0}+\lambda\right)-1}^{y^{*}}\left(\omega^{*}\right) \cap \Pi_{i}\left(\omega^{*}\right)$.

${ }^{48}$ We have that $p\left(\Pi_{i}\left(\omega^{*}\right) \cap \mathcal{F}_{T}^{y^{*}}\left(\omega^{*}\right)>0\right.$ for every $p \in \mathcal{P}$ : this is because we know that there exists $p_{0} \in \mathcal{P}$ with $p_{0}\left(\omega^{*}\right)>0$ which implies, by the prior by prior updating on equilibrium path according to (iii) of Definition 3.3, that $p_{0}\left(\Pi_{i}\left(\omega^{*}\right) \cap \mathcal{F}_{T}^{y^{*}}\left(\omega^{*}\right)\right)$ and hence, again by (iii) of Definition 3.3, we get that for every $p \in \mathcal{P}$ it is $p\left(\Pi_{i}\left(\omega^{*}\right) \cap \mathcal{F}_{T}^{y^{*}}\left(\omega^{*}\right)\right)$.
} 


$$
\begin{gathered}
\left.s\left(y_{i-1, \infty}^{*}\left(\Pi_{i-1}\left(\omega^{\prime}\right), H^{\infty}\left(y^{*}\left(\omega^{\prime}\right)\right)\right), X\right)\right]= \\
\sum_{\omega \in \mathcal{F}_{T}^{y^{*}}\left(\omega^{*}\right)} p(\omega)\left[s\left(y_{i, \infty}^{*}\left(\Pi_{i}\left(\omega^{\prime}\right), H^{\infty}\left(y^{*}\left(\omega^{\prime}\right)\right)\right), X\right)-s\left(y_{i-1, \infty}^{*}\left(\Pi_{i}\left(\omega^{\prime}\right), H^{\infty}\left(y^{*}\left(\omega^{\prime}\right)\right)\right), X\right)\right]= \\
=p\left(\mathcal{F}_{T}^{y^{*}}\left(\omega^{*}\right)\right) E_{p \mid \mathcal{F}_{T}^{y^{*}}\left(\omega^{*}\right)}\left[s\left(y_{i, \infty}^{*}\left(\Pi_{i}\left(\omega^{\prime}\right), H^{\infty}\left(y^{*}\left(\omega^{\prime}\right)\right)\right), X\right)-s\left(y_{i-1, \infty}^{*}\left(\Pi_{i-1}\left(\omega^{\prime}\right), H^{\infty}\left(y^{*}\left(\omega^{\prime}\right)\right)\right), X\right)\right] \geq 0 .
\end{gathered}
$$

Summing for all $i$ we get that on the one hand the sum is zero and on the other hand that every term of the summation is more than or equal to zero. Hence every term of the summation should be zero.

For a similar reason, we conclude that $c_{1}^{\Pi_{1}\left(\omega^{*}\right)}=\ldots=c_{n}^{\Pi_{n}\left(\omega^{*}\right)}=0$ for every $\omega^{*}$, such that there exists $p \in \mathcal{P}$ with $p(\omega)>0$.

\section{Step 3:}

Let assume $i$ and $\omega^{*}$, such that there exists $p \in \mathcal{P}$ with $p\left(\omega^{*}\right)>0$, with

$$
\begin{gathered}
\min _{p \in \mathcal{P}_{\mathcal{F}_{T}^{y^{*}}\left(\omega^{*}\right) \cap \Pi_{i}\left(\omega^{*}\right)}} E_{p}\left[s \left(d_{\mathcal{P}_{\mathcal{F}_{T}^{y^{*}}\left(\omega^{*}\right)}}\left(\Pi_{i}\left(\omega^{*}\right), y_{i-1, \infty}^{*}\left(\Pi_{i}\left(\omega^{\prime}\right), H^{\infty}\left(y^{*}\left(\omega^{\prime}\right)\right)\right), X\right)-\right.\right. \\
\left.s\left(y_{i-1, \infty}^{*}\left(\Pi_{i-1}\left(\omega^{\prime}\right), H^{\infty}\left(y^{*}\left(\omega^{\prime}\right)\right)\right), X\right)\right]>\eta
\end{gathered}
$$

for some $\eta>0$.

Then that is true for all final periods $t>>T$ :

$$
\begin{gathered}
\min _{p \in \mathcal{P}_{\mathcal{F}_{T}^{y^{*}}\left(\omega^{*}\right) \cap \Pi_{i}\left(\omega^{*}\right)}} E_{p}\left[s\left(d_{\mathcal{P}}\left(\mathcal{F}_{T}^{y^{*}}\left(\omega^{*}\right) \cap \Pi_{i}\left(\omega^{*}\right), y_{i-1+n t}^{*}\left(\Pi_{i}\left(\omega^{\prime}\right), H^{i-2+n t}\left(y^{*}\left(\omega^{\prime}\right)\right)\right)\right), X\right)-\right. \\
\left.s\left(y_{i-1+n t}^{*}\left(\Pi_{i-1}\left(\omega^{\prime}\right), H^{i-2+n t}\left(y^{*}\left(\omega^{\prime}\right)\right)\right), X\right)\right]>\eta .
\end{gathered}
$$

Fix such $t_{0}$, for which in the same time the continuation game is less than $\eta>0$. We can do that, because the continuations are converging to zero, as we noticed in Step 2. We can observe that with the same reasoning as in Step 1, but with the only difference to deviate at time $i+n t_{0}$ by playing $d_{\mathcal{P}}\left(\mathcal{F}_{T}^{y^{*}}\left(\omega^{*}\right) \cap \Pi_{i}\left(\omega^{*}\right), y_{i+n t_{0}-1}^{*}\left(\Pi_{i-1}\left(\omega^{*}\right), H^{i+n t_{0}-2}\left(y^{*}\left(\omega^{*}\right)\right)\right)\right)$, which deviation will give for this round utility at least $\eta$, and exactly with the same way as in Step 1 after that, we define an alternative strategy.

In addition, we define $A^{c}=\left\{\omega \in \mathcal{F}_{i+n t_{0}-1}^{y^{*}}\left(\omega^{*}\right) \cap \Pi_{i}\left(\omega^{*}\right): V_{i+n t_{0}+n k}\left(H^{i+n t_{0}-1}\left(y^{*}\left(\omega^{*}\right)\right) \cup\right.\right.$ $\left.H_{i+n t_{0}-1}^{i+n t_{0}+k n-1}\left(\left(y^{\prime i}, y^{*-i}\right)(\omega)\right),\left(y^{*^{i}}, y^{*^{-i}}\right), \omega^{*}\right)<0$ for every $\left.k \geq 1\right\}$. Then for every $\omega \in A^{c}$ we have $f_{t_{0}+1}(\omega)=0$, by definition of $y^{\prime i}$. 
For every $\omega \in A$ take:

$k_{\omega}=\min \left\{k \geq 1: V_{i+n t_{0}+n k}\left(H^{i+n t_{0}-1}\left(y^{*}\left(\omega^{*}\right)\right) \cup H_{i+n t_{0}-1}^{i+n t_{0}+1}\left(\left(y^{\prime i}, y^{*-i}\right)(\omega)\right),\left(y^{*^{i}}, y^{*^{-i}}\right), \omega^{*}\right) \geq\right.$

$0\}$. We know that the minimum is well defined by the definition of $A$.

By the definition of $y^{\prime i}$ we have that for every $\omega \in \mathcal{F}_{T}^{y^{*}}\left(\omega^{*}\right) \cap \Pi_{i}\left(\omega^{*}\right)$ :

$$
\begin{gathered}
V_{i+n t_{0}+n k_{\omega}}\left(H^{i+n t_{0}-1}\left(y^{*}\left(\omega^{*}\right)\right) \cup H_{i+n t_{0}-1}^{i+n t_{0}+k_{\omega} n-1}\left(\left(y^{\prime i}, y^{*-i}\right)(\omega)\right),\left(y^{\prime i}, y^{*^{-i}}\right), \omega^{*}\right)= \\
V_{i+n t_{0}+n k_{\omega}}\left(H^{i+n t_{0}-1}\left(y^{*}\left(\omega^{*}\right)\right) \cup H_{i+n t_{0}-1}^{i+n t_{0}+k_{\omega} n-1}\left(\left(y^{\prime i}, y^{*-i}\right)(\omega)\right),\left(y^{*^{i}}, y^{*^{-i}}\right), \omega^{*}\right) \geq 0
\end{gathered}
$$

and for the preceding information sets at rounds $t_{0}+1, \ldots, t_{0}+k_{\omega}$ the deviation action of $i$ is playing whatever the previous, but for round $t_{0}$ where trader is playing $d_{\mathcal{P}}\left(\mathcal{F}_{T}^{y^{*}}\left(\omega^{*}\right) \cap\right.$ $\left.\Pi_{i}\left(\omega^{*}\right), y_{i+n t_{0}-1}^{*}\left(\Pi_{i-1}\left(\omega^{*}\right), H^{i+n t_{0}-2}\left(y^{*}\left(\omega^{*}\right)\right)\right)\right)$.

For every $\omega \in A$ set:

$A_{\omega}=\left\{\omega^{\prime} \in A: H^{i+n t_{0}-1}\left(y^{*}\left(\omega^{*}\right)\right) \cup H_{i-1+n t_{0}}^{i-1+n t_{0}+n k_{\omega}}\left(\left(y^{i}, y^{*-i}\right)\left(\omega^{\prime}\right)\right)=H^{i+n t_{0}-1}\left(y^{*}\left(\omega^{*}\right)\right) \cup\right.$ $\left.H_{i-1+n t_{0}}^{i-1+n t_{\omega}}\left(\left(y^{\prime i}, y^{*-i}\right)(\omega)\right)\right\}$.

Intuitively, this is the set of states that $i$ thinks are possible to happen, when the realised state is $\omega^{*}$, the round is $\left(t_{0}+k_{\omega}\right)$ and the history is $H^{i+n t_{0}-1}\left(y^{*}\left(\omega^{*}\right)\right) \cup H_{i-1+n t_{0}}^{i-1+n k_{\omega}}\left(\left(y^{\prime i}, y^{*-i}\right)(\omega)\right)$. In fact, these sets form a partition of the set $A$. Name the partition $A_{\omega_{1}}, \ldots, A_{\omega_{m}}$. Essentially, it is the joint information between the public information created by the corresponding history and $i$ 's private signal.

Next, we can observe that:

$$
\begin{gathered}
V_{i+n t_{0}}\left(H^{i+n t_{0}-1}\left(y^{*}\left(\omega^{*}\right)\right),\left(y^{\prime i}, y^{*-i}\right), \omega^{*}\right)= \\
E_{p^{*}}\left[f_{t_{0}}\right]= \\
E_{p^{*}}\left[s\left(d_{\mathcal{P}}\left(\mathcal{F}_{T}^{y^{*}}\left(\omega^{*}\right) \cap \Pi_{i}\left(\omega^{*}\right), y_{i+n t_{0}-1}^{*}\left(\Pi_{i-1}\left(\omega^{*}\right), H^{i+n t_{0}-2}\left(y^{*}\left(\omega^{*}\right)\right)\right)\right), X\right)-\right. \\
\left.s\left(y_{i-1+n t}^{*}\left(\Pi_{i-1}\left(\omega^{\prime}\right), H^{i-2+n t}\left(y^{*}\left(\omega^{\prime}\right)\right)\right), X\right)\right]+\sum_{\omega \in A} \beta^{n} f_{t_{0}+1}(\omega) p^{*}(\omega)
\end{gathered}
$$

for some $p^{*} \in \mathcal{P}_{\mathcal{F}_{i+n t_{0}-1}^{y^{*}}\left(\omega^{*}\right) \cap \Pi_{i}\left(\omega^{*}\right)}{ }^{49}$

But

$$
\sum_{\omega \in A} \beta^{n} f_{t_{0}+1}(\omega) p^{*}(\omega)=
$$

${ }^{49}$ Here we should have written $E_{p^{*}}\left[s\left(d_{\mathcal{P}}\left(\mathcal{F}_{T}^{y^{*}}\left(\omega^{*}\right) \cap \Pi_{i}\left(\omega^{*}\right), y_{i+n t_{0}-1}^{*}\left(\Pi_{i-1}\left(\omega^{*}\right), H^{i+n t_{0}-2}\left(y^{*}\left(\omega^{*}\right)\right)\right)\right), X\right)-\right.$ $\left.s\left(y_{i-1+n t}^{*}\left(\Pi_{i-1}\left(\omega^{\prime}\right), H^{i-2+n t}\left(y^{*}\left(\omega^{*}\right)\right)\right), X\right)\right] \quad$ instead $\quad$ of $\quad E_{p^{*}}\left[s\left(d_{\mathcal{P}}\left(\mathcal{F}_{T}^{y^{*}}\left(\omega^{*}\right) \quad \cap\right.\right.\right.$ $\left.\left.\left.\Pi_{i}\left(\omega^{*}\right), y_{i+n t_{0}-1}^{*}\left(\Pi_{i-1}\left(\omega^{*}\right), H^{i+n t_{0}-2}\left(y^{*}\left(\omega^{*}\right)\right)\right)\right), X\right)-s\left(y_{i-1+n t}^{*}\left(\Pi_{i-1}\left(\omega^{\prime}\right), H^{i-2+n t}\left(y^{*}\left(\omega^{\prime}\right)\right)\right), X\right)\right]$. However, we can do that because for every $\omega^{\prime} \in \mathcal{F}_{T}^{y^{*}}\left(\omega^{*}\right) \cap \Pi_{i}\left(\omega^{*}\right)$ and $t>T$ it is $H^{i-2+n t}\left(y^{*}\left(\omega^{\prime}\right)\right)=H^{i-2+n t}\left(y^{*}\left(\omega^{*}\right)\right)$, by the definition of $\mathcal{F}_{T}^{y^{*}}\left(\omega^{*}\right)$. 
$p^{*}\left(A_{\omega_{1}}\right) \sum_{\omega \in A_{\omega_{1}}} \beta^{n k_{\omega_{1}}} f_{t_{0}+k \omega_{1}}(\omega) \frac{p^{*}(\omega)}{p^{*}\left(A_{\omega_{1}}\right)}+\ldots+p^{*}\left(A_{\omega_{m}}\right) \sum_{\omega \in A_{\omega_{m}}} \beta^{n k_{\omega_{m}}} f_{t_{0}+k \omega_{m}}(\omega) \frac{p^{*}(\omega)}{p^{*}\left(A_{\omega_{m}}\right)}$.

In addition, for every $\omega_{1}, \ldots, \omega_{m}$ it holds that:

$$
\begin{aligned}
& \sum_{\omega \in A_{\omega_{j}}} \beta^{n k_{\omega_{j}}} f_{t_{0}+k \omega_{j}}(\omega) \frac{p^{*}(\omega)}{p^{*}\left(A_{\omega_{j}}\right)} \geq \\
& \beta^{n k_{\omega_{j}}} V_{i+n t_{0}+n k_{\omega_{j}}}\left(H^{i+n t_{0}-1}\left(y^{*}\left(\omega^{*}\right)\right) \cup H_{i+n t_{0}-1}^{i+n t_{0}+k_{\omega_{j}} n-1}\left(\left(y^{\prime i}, y^{*-i}\right)\left(\omega_{j}\right)\right),\left(y^{\prime i}, y^{*^{-i}}\right), \omega^{*}\right)= \\
& \beta^{n k_{\omega_{j}}} V_{i+n t_{0}+n k_{\omega_{j}}}\left(H^{i+n t_{0}-1}\left(y^{*}\left(\omega^{*}\right)\right) \cup H_{i+n t_{0}-1}^{i+n t_{0}+k_{\omega} n-1}\left(\left(y^{\prime i}, y^{*-i}\right)\left(\omega_{j}\right)\right),\left(y^{*^{i}}, y^{*^{-i}}\right), \omega^{*}\right) \geq 0 .
\end{aligned}
$$

Hence

$$
V_{i+n t_{0}}\left(H^{i+n t_{0}-1}\left(y^{*}\left(\omega^{*}\right)\right),\left(y^{\prime i}, y^{*-i}\right), \omega^{*}\right) \geq \eta \cdot{ }^{50}
$$

Assume now that we are on deviation strategy's path at round, say $t_{0}+\lambda$, with $\lambda \geq 1$. If for every $\omega \in \mathcal{F}_{i+n\left(t_{0}+\lambda\right)-1}^{y^{*}}\left(\omega^{*}\right) \cap \Pi_{i}\left(\omega^{*}\right)$ it is $k_{\omega} \leq t_{0}+\lambda$ then the deviation strategy in the continuation is the same as the equilibrium's one. ${ }^{51}$ Otherwise, we can apply exactly the reasoning of Step 1 for the corresponding information set.

In addition, outside the deviation path we have that the payoffs are the same, by its definition, too. Hence, we get a contradiction, because $y^{*}$ is revision proof.

We can conclude a contradiction because $y_{i}^{*}$ is revision proof equilibrium.

Therefore, it should be the case that for every $i$ and every $\omega^{*}$, for which there exists $p \in \mathcal{P}$ with $p\left(\omega^{*}\right)>0$, it is:

$$
\begin{gathered}
\min _{p \in \mathcal{P}_{\mathcal{F}_{T}^{y^{*}}\left(\omega^{*}\right) \cap \Pi_{i}\left(\omega^{*}\right)}} E_{p}\left[s \left(d_{\mathcal{P}_{\mathcal{F}_{T}^{y^{*}}\left(\omega^{*}\right)}}\left(\Pi_{i}\left(\omega^{*}\right), y_{i-1, \infty}^{*}\left(\Pi_{i}\left(\omega^{\prime}\right), H^{\infty}\left(y^{*}\left(\omega^{\prime}\right)\right)\right), X\right)-\right.\right. \\
\left.s\left(y_{i-1, \infty}^{*}\left(\Pi_{i-1}\left(\omega^{\prime}\right), H^{\infty}\left(y^{*}\left(\omega^{\prime}\right)\right)\right), X\right)\right]=0 .
\end{gathered}
$$

For every $i$ the limit strategies are constant over $\mathcal{F}_{T}^{y^{*}}\left(\omega^{*}\right)$ and hence

$$
y_{i, \infty}^{*}\left(\Pi_{i}\left(\omega^{*}\right), H^{\infty}\left(y^{*}\left(\omega^{*}\right)\right)\right)=y_{i, \infty}^{*}\left(\Pi_{i}\left(\omega^{\prime}\right), H^{\infty}\left(y^{*}\left(\omega^{\prime}\right)\right)\right)
$$

for every $\omega^{\prime} \in \mathcal{F}_{T}^{y^{*}}\left(\omega^{*}\right)$ with $p\left(\omega^{\prime}\right)>0$ for some $p \in \mathcal{P}$.

\footnotetext{
${ }^{50}$ In this argument we use Definition $3.3($ iii): the deviator (i.e. $i$ trader) updates prior by prior.

${ }^{51}$ Notice here that if there exists $\omega \in \mathcal{F}_{i+n\left(t_{0}+\lambda\right)-1}^{y^{*}}\left(\omega^{*}\right) \cap \Pi_{i}\left(\omega^{*}\right)$ it is $k_{\omega} \leq t_{0}+\lambda$ then the same applies for every $\omega \in \mathcal{F}_{i+n\left(t_{0}+\lambda\right)-1}^{y^{*}}\left(\omega^{*}\right) \cap \Pi_{i}\left(\omega^{*}\right)$.
} 
Therefore, for every $\omega^{*}$, for which there exists $p \in \mathcal{P}$ with $p\left(\omega^{*}\right)>0$, it is:

(1) $d_{\mathcal{P}_{\mathcal{F}_{T}^{y^{*}}\left(\omega^{*}\right)}}\left(\Pi_{i}\left(\omega^{*}\right), y_{i-1, \infty}^{*}\left(\Pi_{i-1}\left(\omega^{*}\right), H^{\infty}\left(y^{*}\left(\omega^{*}\right)\right)\right)\right)=y_{i-1, \infty}^{*}\left(\Pi_{i-1}\left(\omega^{*}\right), H^{\infty}\left(y^{*}\left(\omega^{*}\right)\right)\right)$.

(2) $c_{i}^{\Pi_{i}\left(\omega^{*}\right)}=0$, as well, and hence by the uniqueness of the $\underset{y \in Y}{\operatorname{argmax}} \min _{\mathcal{F}_{T}^{y^{*}}\left(\omega^{*}\right) \cap \Pi_{i}\left(\omega^{*}\right)} E_{p}\left[s(y, X(\omega))-s\left(y_{i-1, \infty}^{*}\left(\Pi_{i-1}\left(\omega^{\prime}\right), H^{\infty}\left(y^{*}\left(\omega^{\prime}\right)\right)\right), X(\omega)\right)\right]$ (using arguments of Lemma 2.3) it should be the case that for every $i$ it is $y_{i, \infty}^{*}\left(\Pi_{i}\left(\omega^{*}\right), H^{\infty}\left(y^{*}\left(\omega^{*}\right)\right)\right)=$ $d_{\mathcal{P}} \overline{\mathcal{F}}_{T}^{y^{*}\left(\omega^{*}\right)}\left(\Pi_{i}\left(\omega^{*}\right), y_{i-1, \infty}^{*}\left(\Pi_{i}\left(\omega^{*}\right), H^{\infty}\left(y^{*}\left(\omega^{*}\right)\right)\right)\right)$.

Then defining $V_{i}=\left\{y_{i, \infty}^{*}\left(\Pi_{i}\left(\omega^{*}\right), H^{\infty}\left(y^{*}\left(\omega^{*}\right)\right)\right)\right\}$ and using the assumption of $X$ being separable under ambiguity, for $\mathcal{P}_{\mathcal{F}_{T}^{y^{*}}\left(\omega^{*}\right)}$, we conclude that information gets aggregated. ${ }^{52}$

Here we prove Theorem $3.6($ ii) :

Proof. Take a non-separable under ambiguity security under $\Pi$ and $s$. Then there exists $\mathcal{P} \subseteq \Delta(\Omega)$ convex and compact whose every prior gives positive probability in each element of $\Pi$, and $\mathcal{O}$ and $v \in \mathbb{R}$ such that:

for every $i=1, \ldots, n$ and for every $\omega$, such that there exists $p \in \mathcal{P}$ with $p(\omega)>0$, it is $d_{\mathcal{P}}\left(\Pi_{i}(\omega), v\right)=v$.

We will show that if MM announcement is $v$ then there exists a converging revision proof equilibrium in which information does not get aggregated.

Define the assessment $\left(y^{*}, \mathcal{P}\right)$, where $y^{*}$ is the following strategy profile:

every player $i$ predicts $v$ after any history. ${ }^{53}$

As far as the beliefs are concerned, on equilibrium path the prior by prior updating is used and for the rest off equilibrium information sets the beliefs are given by $\mathcal{P}$ over the information set with prior by prior updating whenever possible.

Given that initially the MM announces $v$, the strategic traders are essentially playing their myopic strategy because for every $i$ and for every $\omega$, such that there exists $p \in \mathcal{P}$ with $p(\omega)>0$, it is $d_{\mathcal{P}}\left(\Pi_{i}(\omega), v\right)=v$ or in other words their strategic actions solves the $\operatorname{maxmin}_{y \in Y} E_{p \in \mathcal{P}} E_{p \mid \Pi_{1}\left(\omega^{*}\right)}[s(y, X(\omega))-s(v, X(\omega))]$.

\footnotetext{
${ }^{52}$ By Lemma A.1 and Definition 3.3 (iii) we know that the priors of the set $\mathcal{P}_{\mathcal{F}_{T}^{y^{*}}\left(\omega^{*}\right)}$ are mutually absolute continuous with respect to $\Pi_{i}$, for $i=1, \ldots, n$.

${ }^{53}$ We observe that the equilibrium profile defined satisfies the converging strategies assumption.
} 
Next we can observe that the maximum continuation payoff for an agent $i$ after history $H^{t}$, given that $-i$ follow the $y^{*}$, is $^{54}$ :

$$
\begin{gathered}
\min _{p \in \mathcal{P}_{H^{t}}} E_{p \mid \Pi_{i}\left(\omega^{*}\right)}\left[\sum_{k=t}^{\infty} \beta^{n k-n t}\left(s\left(E_{p \mid \Pi_{i}\left(\omega^{*}\right)}, X(\omega)\right)-s(v, X(\omega))\right)\right]= \\
=\min _{p \in \mathcal{P}_{H^{t}}} \frac{E_{p \mid \Pi_{i}(\omega)}\left[s\left(E_{p \mid \Pi_{i}\left(\omega^{*}\right)}, X(\omega)\right)-s(v, X(\omega))\right]}{1-\beta^{n}}= \\
=\min _{p \in \mathcal{P}_{H^{t}}} \max _{y \in Y} \frac{E_{p \mid \Pi_{i}(\omega)}[s(y, X(\omega))-s(v, X(\omega))]}{1-\beta^{n}} .
\end{gathered}
$$

However, by the arguments of Lemma 2.3, we have that

$\min _{p \in \mathcal{P}_{H^{t}}} \max _{y \in Y} E_{p \mid \Pi_{i}(\omega)}[s(y, X(\omega))-s(v, X(\omega))]=\max _{y \in Y} \min _{p \in \mathcal{P}_{H^{t}}} E_{p \mid \Pi_{i}(\omega)}[s(y, X(\omega))-s(v, X(\omega))]$.

In addition, by definition of $y^{*}$ we have $\mathcal{P}_{H^{t}}=\mathcal{P}$ (i.e. no essential information refinement ever happens). Therefore, it cannot be alternative strategy profile that gives at some round and under some history strictly better payoff than $y^{*}$, and hence we conclude that it is revision proof equilibrium.

\footnotetext{
${ }^{54}$ In order to understand the maximum continuation payoff for agent $i$ we need firstly to observe that because the equilibrium actions of the other agents are $v$ whatever the history or the state is, then the strategy for agent $i$ can be written as, for every $k \geq t$ and $\omega^{\prime} \in \Pi_{i}(\omega)$, $y_{i+n k}\left(\Pi_{i}(\omega), H^{i-1+n t} \cup H_{i-1+n t}^{i-1+n k}\left(\left(y_{i}, y_{-i}^{*}\right)\left(\omega^{\prime}\right)\right)\right)=y_{i+n k}\left(\Pi_{i}(\omega), H^{i-1+n t} \cup H_{i-1+n t}^{i-1+n k}\left(\left(y_{i}, y_{-i}^{*}\right)(\omega)\right)\right)$ which we can denote for simplicity by $y_{i+n k}$ because $\omega$ can be thought as given.

Secondly, we claim that $\max _{\left(y_{i+n k}\right)_{k} \in Y^{\mathbb{N}} p \in \mathcal{P}_{H^{t}(\omega)}} E_{p}\left[\sum_{k=t}^{\infty} \beta^{n k-n t}\left(s\left(y_{i+n k}, X\right)-s(v, X)\right)\right] \leq$ $\max _{y \in Y} \min _{p \in \mathcal{P}_{H^{t}(\omega)}} E_{p}\left[\sum_{k=t}^{\infty} \beta^{n k-n t}(s(y, X)-s(v, X))\right]$. Indeed, by Lemma 2.3 we can see that $\max _{y \in Y} \min _{p \in \mathcal{P}_{H^{t}(\omega)}} E_{p}\left[\sum_{k=t}^{\infty} \beta^{n k-n t}(s(y, X)-s(v, X))\right]=E_{p^{*}}\left[\sum_{k=t}^{\infty} \beta^{n k-n t}\left(s\left(E_{p^{*}}[X], X\right)-s(v, X)\right)\right]$ for some $p^{*} \in \mathcal{P}_{H^{t}(\omega)}$. Denote by $\left(y_{i+n k}\right)_{k}$ a solution of the left hand side "maxmin" problem. Then $\min _{p \in \mathcal{P}_{H^{t}(\omega)}} E_{p}\left[\sum_{k=t}^{\infty} \beta^{n k-n t}\left(s\left(y_{i+n k}, X\right)-s(v, X)\right)\right] \leq E_{p^{*}}\left[\sum_{k=t}^{\infty} \beta^{n k-n t}\left(s\left(y_{i+n k}, X\right)-s(v, X)\right)\right] \leq$ $E_{p^{*}}\left[\sum_{k=t}^{\infty} \beta^{n k-n t}\left(s\left(E_{p^{*}}[X], X\right)-s(v, X)\right)\right]$. In other words, what it have just proved is that in order to maximise your continuation expected payoff, given that the other players are playing at any case $v$, you need to consider only those continuation profiles that prescribe the same action for every future information set.
} 



\section{Appendix D}

Below we prove Theorem 3.7.

Proof. Let $\mathrm{X}$ to be the separable under ambiguity security and $y^{*}$ the equilibrium profile. By Theorem 3.6 we know that for every state $\omega^{*}$ that can be realised there exists some time $T$ such that $\mathcal{F}_{T}^{y^{*}}\left(\omega^{*}\right)$ is the finest public information and for every $\omega \in \mathcal{F}_{T}^{y^{*}}\left(\omega^{*}\right)$ it is $X(\omega)=X\left(\omega^{*}\right)$. Therefore, we can conclude that if traders are myopic, for a round latter than $T$, then they should announce $X\left(\omega^{*}\right)$. In addition, by Theorem 3.5 again we know that information gets aggregated and thus $y_{t}^{*} \longrightarrow_{t>T} X\left(\omega^{*}\right)$, where $y_{t}^{*}=y_{(t m o d n)+n(t-1)}^{*}\left(\Pi_{(\operatorname{tmodn})}\left(\omega^{*}\right), H^{t-1}\left(y^{*}\left(\omega^{*}\right)\right)\right)$. In addition, $s_{t}^{*}=s\left(y_{t}^{*}, X\left(\omega^{*}\right)\right) \longrightarrow$ $s^{*}=s\left(X\left(\omega^{*}\right), X\left(\omega^{*}\right)\right.$.

Assume that there exists $y_{t}^{*}$, for $t>T$, such that $y_{t}^{*} \neq X\left(\omega^{*}\right)$. Hence it is $s_{t}^{*} \neq s^{*}$. Then we can define

$$
t_{0}=\min \left\{m: \text { for every } k \geq m \text { it is }\left|s_{k}^{*}-s^{*}\right| \leq \epsilon\right\}
$$

with $0<\epsilon<\left|s^{*}-s_{t}^{*}\right|=s^{*}-s_{t}^{*}$. ${ }^{55}$ We have that $t_{0}-1 \geq t>T$.

Without loss in generality, assume that $y_{t_{0}-1}^{*}$ is actually the $y_{i+n t_{1}}^{*}$ which in turn corresponds to $s_{i+n t_{1}}^{*}$. By its definition $\left|s^{*}-s_{t_{0}-1}^{*}\right|=\left|s^{*}-s_{i+n t_{1}}^{*}\right|=s^{*}-s_{i+n t_{1}}^{*}>\epsilon$.

By definition of $t_{0}$ we have that for every $k>t_{1}$ it is $\left|s_{i+n k}^{*}-s_{i-1+n k}^{*}\right| \leq 2 \epsilon$. Hence for $\beta^{n}<\frac{1}{3}$ we get that:

$$
\begin{gathered}
\beta^{n} \sum_{k=t_{1}+1}^{\infty} \beta^{n\left(k-t_{1}-1\right)}\left(s_{i+n k}^{*}-s_{i-1+n k}^{*}\right) \leq \\
\beta^{n} \sum_{k=t_{1}+1}^{\infty} \beta^{n\left(k-t_{1}-1\right)}\left|s_{i+n k}^{*}-s_{i-1+n k}^{*}\right| \leq \\
\frac{\beta^{n}}{1-\beta^{n}} 2 \epsilon \leq \epsilon .
\end{gathered}
$$

${ }^{55}$ This is because $s^{*}$ is the score for the true state when the prediction is the true state, and $X(\omega) \neq y_{t}^{*}$. 
Therefore:

$$
\begin{gathered}
s_{i+n t_{1}}^{*}-s_{i-1+n t_{1}}^{*}+\beta^{n} \sum_{k=t_{1}+1}^{\infty} \beta^{n\left(k-t_{1}-1\right)}\left(s_{i+n k}^{*}-s_{i-1+n k}^{*}\right) \leq \\
s_{i+n t_{1}}^{*}-s_{i-1+n t_{1}}^{*}+\epsilon<s^{*}-s_{i+n t_{1}}^{*}+s_{i+n t_{1}}^{*}-s_{i-1+n t_{1}}^{*}=s^{*}-s_{i-1+n t_{1}}^{*} .
\end{gathered}
$$

By definition of $\mathcal{F}_{T}^{*}\left(\omega^{*}\right)$ and because $X(\omega)=X\left(\omega^{*}\right)$ for every $\omega \in \mathcal{F}_{T}^{*}\left(\omega^{*}\right)$ we have:

$$
\begin{gathered}
V_{i+n t_{1}}\left(H^{i-1+n t_{1}}\left(y^{*}\left(\omega^{*}\right)\right), y^{*}, \omega^{*}\right)= \\
s_{i+n t_{1}}^{*}-s_{i-1+n t_{1}}^{*}+\beta^{n} \sum_{k=t_{1}+1}^{\infty} \beta^{n\left(k-t_{1}-1\right)}\left(s_{i+n k}^{*}-s_{i-1+n k}^{*}\right)
\end{gathered}
$$

and

$$
\begin{gathered}
\min _{p \in \mathcal{P}_{\mathcal{F}_{T}^{y^{*}}\left(\omega^{*}\right) \cap \Pi_{i}\left(\omega^{*}\right)}} E_{p}\left[s \left(d_{\mathcal{P}_{\mathcal{F}_{T}^{y^{*}}\left(\omega^{*}\right)}}\left(\Pi_{i}\left(\omega^{*}\right), y_{i-1+n t_{1}}^{*}\left(\Pi_{i}\left(\omega^{\prime}\right), H^{i-2+n t_{2}}\left(y^{*}\left(\omega^{\prime}\right)\right)\right), X\right)-\right.\right. \\
\left.s\left(y_{i-1+n t_{1}}^{*}\left(\Pi_{i}\left(\omega^{\prime}\right), H^{i-2+n t_{2}}\left(y^{*}\left(\omega^{\prime}\right)\right)\right), X\right)\right]= \\
s^{*}-s_{i-1+n t_{1}}^{*} .
\end{gathered}
$$

Therefore by the first argument in Step 3 of Theorem 3.6 we get a contradiction with $y^{*}$ being revision proof.

Next we prove Theorem 3.8:

Proof. By Theorem 3.6 we know that for every state $\omega^{*}$ that can be realised it is $X(\omega)=$ $X\left(\omega^{*}\right)$ for every $\omega \in \mathcal{F}_{n}^{y^{*}}\left(\omega^{*}\right)$.

From Theorem 3.7 we know that after $n+1$-th announcement (including $n+1$ ) every trader plays myopically, hence for every state $\omega^{*}$ that can be realised and every $i=1, \ldots, n$ they predict $y_{i+k n}^{*}\left(\Pi_{i}\left(\omega^{*}\right), H^{i-1+k n}\left(y^{*}\left(\omega^{*}\right)\right)\right)=X\left(\omega^{*}\right)$, for every $k=1,2, \ldots$

The utility of trader $i=1, \ldots, n$, divided by $\beta^{i}$, at their information set when $k=0$ is as follows:

$\min _{p \in \mathcal{P}_{\mathcal{F}_{i-1}^{y^{*}}\left(\omega^{*}\right) \cap \Pi_{i}(\omega)}} E_{p}\left[\left(s\left(y_{i}^{*}\left(\Pi_{i}\left(\omega^{*}\right), H^{i-1}\left(y^{*}\left(\omega^{\prime}\right)\right)\right), X\right)-s\left(y_{i-1}^{*}\left(\Pi_{i-1}\left(\omega^{\prime}\right), H^{i-2}\left(y^{*}\left(\omega^{\prime}\right)\right)\right), X\right)\right)+\right.$ $\left.\sum_{k=1}^{\infty} \beta^{n k}\left(s\left(y_{i+n k}^{*}\left(\Pi_{i}\left(\omega^{*}\right), H^{i-1+n k}\left(y^{*}\left(\omega^{\prime}\right)\right)\right), X\right)-s\left(y_{i-1+n k}^{*}\left(\Pi_{i-1}\left(\omega^{\prime}\right), H^{i-2+n k}\left(y^{*}\left(\omega^{\prime}\right)\right)\right), X\right)\right)\right]$. 
Therefore, for every $i=2, \ldots, n$ the utility turns out to be:

$\min _{p \in \mathcal{P}} E_{\mathcal{F}_{i-1}^{y^{*}}\left(\omega^{*}\right) \cap \Pi_{i}(\omega)}\left[\left(s\left(y_{i}^{*}\left(\Pi_{i}\left(\omega^{*}\right), H^{i-1}\left(y^{*}\left(\omega^{\prime}\right)\right)\right), X\right)-s\left(y_{i-1}^{*}\left(\Pi_{i-1}\left(\omega^{\prime}\right), H^{i-2}\left(y^{*}\left(\omega^{\prime}\right)\right)\right), X\right)\right)\right]$.

This is because for every state $\omega^{*}$ that can be realised it is $y_{i+k n}^{*}\left(\Pi_{i}\left(\omega^{*}\right), H^{i-1+k n}\left(y^{*}\left(\omega^{*}\right)\right)\right)=$ $X\left(\omega^{*}\right)$ for every $i=1, \ldots, n$, for every $k=1,2, \ldots$

If there exists a trader $i \in\{2, \ldots, n\}$ that in his first round action does not play myopically, then if she would have played myopically her utility when $k=0$ would be higher than the utility from her continuation game (on equilibrium path). Hence, with the same argument as in Step 3 of Theorem 3.6 we get a contradiction with $y^{*}$ being revision proof. Hence, every $i \in\{2, \ldots, n\}$ plays myopically for every state that can be realised.

The same applies for $i=1$ as well. However we need the result of the previous paragraph and the following argument to conclude it: for every $\omega^{*}$, with $p\left(\omega^{*}\right)>0$ for some $p \in \mathcal{P}$, we have that for every $\omega \in \mathcal{F}_{n+1}^{y^{*}}\left(\omega^{*}\right)$ it is $X(\omega)=X\left(\omega^{*}\right)$ and that $\mathcal{F}_{n}^{y^{*}}\left(\omega^{*}\right) \supseteq$ $\Pi_{n}\left(\omega^{*}\right) \cap \mathcal{F}_{n-1}^{y^{*}}\left(\omega^{*}\right)$ therefore $X(\omega)=X\left(\omega^{*}\right)$ for every $\omega \in \Pi_{n}\left(\omega^{*}\right) \cap \mathcal{F}_{n-1}^{y^{*}}\left(\omega^{*}\right)$ (hence $n$-th trader's myopic prediction is essentially $X\left(\omega^{*}\right)$, therefore trader's 1 continuation is zero). 



\section{Appendix E}

The following tables illustrate the experimental data as used in the permutation tests.

\begin{tabular}{|c|c|c|c|c|c|c|c|c|c|c|c|c|c|c|c|}
\hline \multicolumn{6}{|c|}{ Red Ball } & \multicolumn{5}{|c|}{ Green Ball } & \multicolumn{5}{|c|}{ Yellow Ball } \\
\hline & Last Round & ast 2 Rounds & ast 3 Round & last 4 Rounds & Average & & Last Round & ast 2 Roun & ast 3 Roun & Average & & Last Rounc & ast 2 Roun & Last 3 Rounds & Average \\
\hline Risk & 25 & 52.5 & 43.33 & 48.75 & 42.395 & Risk & 27.5 & 28.125 & 22.08 & 25.9017 & Risk & 2.5 & 9.75 & 15.25 & 9.166666667 \\
\hline Risk & 6.25 & 3.875 & 9.08 & 15 & 8.55125 & Risk & 0 & 15.625 & 11 & 8.875 & Risk & 25 & 14.125 & 18.58333333 & 19.23611111 \\
\hline Risk & 25.75 & 30.37 & 33.58 & 41.44 & 32.785 & Risk & 1.25 & 13.125 & 18.75 & 11.0417 & Risk & 14.25 & 37.75 & 42.41666667 & 31.47222222 \\
\hline Risk & 12.5 & 12.625 & 12.66 & 34.5 & 18.07125 & Risk & 62.5 & 47.5 & 54.16 & 54.72 & Risk & 20 & 11.5 & 11.83333333 & 14.444444444 \\
\hline Amb & 67.5 & 68.75 & 67.83 & 68.125 & 68.05125 & Amb & 52.5 & 56.25 & 50.41 & 53.0533 & Amb & 14 & 23.275 & 29.16666667 & 22.147222222 \\
\hline Amb & 48.5 & 24.875 & 18 & 24.8125 & 29.046875 & Amb & 47 & 33.9 & 20.5 & 33.8 & Amb & 50.25 & 31.375 & 36.75 & 39.45833333 \\
\hline Amb & 44 & 63.25 & 63 & 57.875 & 57.03125 & Amb & 35 & 36.25 & 28.33 & 33.1933 & Amb & 40.75 & 36.625 & 35.25 & 37.54166667 \\
\hline Amb & 82.5 & 59.375 & 55.41 & 53.4375 & 62.680625 & Amb & 2.5 & 16.25 & 29.16 & 15.97 & Amb & 25 & 12.5 & 8.333333333 & 15.27777778 \\
\hline p-values & $0.01461^{* * *}$ & $0.04336 *$ & $0.0575^{*}$ & 0.11523 & $0.04297^{* *}$ & p-values & 0.6016 & 0.4284 & 0.57287 & 0.45791 & p-values & $0.0986^{*}$ & 0.2002 & 0.2863 & 0.1011 \\
\hline
\end{tabular}

Figure E.1: Experimental Data for Security X.

\begin{tabular}{|c|c|c|c|c|c|c|c|c|c|c|c|c|c|c|c|}
\hline \multicolumn{6}{|c|}{ Red Ball } & \multicolumn{5}{|c|}{ Green Ball } & \multicolumn{5}{|c|}{ Yellow Ball } \\
\hline & Last Rounc & sst 2 Rounds & ast 3 Round & ast 4 Rounds & Average & & Last Roun & st 2 Roun & st 3 Rour & Average & & Last Roun & st 2 Rounds & Last 3 Rounds & Average \\
\hline Risk & 33.33 & 26.66 & 17.77 & 16.66 & 23,605 & Risk & 0 & 5.16 & 3.44 & 2.86667 & Risk & 0 & 8.33 & 16.66666667 & 8.332222222 \\
\hline Risk & 33 & 32.66 & 32.22 & 27.083 & 31.24075 & Risk & 16.66 & 9.16 & 6.22 & 10.68 & Risk & 47.33 & 47.83 & 42.44444444 & 45.86814815 \\
\hline Risk & 0 & 12.5 & 10.833 & 8.125 & 7.8645 & Risk & 0 & 0 & 0 & 0 & Risk & 0 & 0 & 1.25 & 0.416666667 \\
\hline Risk & 0 & 0 & 0 & 0 & 0 & Risk & 0 & 0 & 0 & 0 & Risk & 0 & 0 & 5.416666667 & 1.805555556 \\
\hline$A m b$ & 0 & 0 & 0 & 0 & 0 & $A m b$ & 0 & 3.875 & 2.58 & 2.15167 & $A m b$ & 0 & 0.125 & 0.083333333 & 0.069444444 \\
\hline$A m b$ & 0 & 1.625 & 9.833 & 10.5 & 5.4895 & $A m b$ & 0.25 & 0.125 & 0.5 & 0.29167 & $A m b$ & 26.25 & 14.5 & 18.16666667 & 19.63888889 \\
\hline$A m b$ & 7.5 & 4 & 20.1666 & 15.125 & 11.6979 & $A m b$ & 0 & 5 & 3.75 & 2.91667 & $A m b$ & 47.5 & 31.25 & 29.16666667 & 35.97222222 \\
\hline Amb & 6 & 3.375 & 2.416 & 5.1875 & 4.244625 & $A m b$ & 15.25 & 14 & 9.33 & 12.86 & $A m b$ & 19.5 & 21.75 & 15.66666667 & 18.97222222 \\
\hline$p$-values & 0.4268 & $0.071^{*}$ & 0.48375 & 0.48703 & 0.283 & & 1 & 0.6867 & 0.485 & 0.6002 & & 0.5486 & 0.8571 & 0.9152 & 0.7131 \\
\hline
\end{tabular}

Figure E.2: Experimental Data for Security Y. 



\section{Bibliography}

Ales, L. \& Sleet, C. (2014), 'Revision proofness', Journal of Economic Theory 152, 324355.

Aumann, R. J. (1976), 'Agreeing to disagree', The annals of statistics pp. 1236-1239.

Azrieli, Y. \& Teper, R. (2011), 'Uncertainty aversion and equilibrium existence in games with incomplete information', Games and Economic Behavior 73(2), 310-317.

Bacharach, M. (1985), 'Some extensions of a claim of aumann in an axiomatic model of knowledge', Journal of Economic Theory 37(1), 167-190.

Battigalli, P., Cerreia-Vioglio, S., Maccheroni, F. \& Marinacci, M. (2015), 'Selfconfirming equilibrium and model uncertainty', The American Economic Review 105(2), 646-677.

Berge, C. (1963), Topological Spaces: including a treatment of multi-valued functions, vector spaces, and convexity, Courier Dover Publications.

Bonanno, G. (2013), 'Agm-consistency and perfect bayesian equilibrium. part i: definition and properties', International Journal of Game Theory 42(3), 567-592.

Bose, S. \& Daripa, A. (2009), 'A dynamic mechanism and surplus extraction under ambiguity', Journal of Economic theory 144(5), 2084-2114.

Bose, S. \& Renou, L. (2011), 'Mechanism design with ambiguous communication devices', Available at SSRN 1868132.

Cabral, L., Ozbay, E. Y. \& Schotter, A. (2014), 'Intrinsic and instrumental reciprocity: An experimental study', Games and Economic Behavior 87, 100-121.

Carvajal, A. \& Correia-da Silva, J. (2010), Agreeing to disagree with multiple priors, Technical report, Universidade do Porto, Faculdade de Economia do Porto.

Cave, J. A. (1983), 'Learning to agree', Economics Letters 12(2), 147-152.

Chambers, C. P. (2008), 'Proper scoring rules for general decision models', Games and Economic Behavior 63(1), 32-40. 
Chateauneuf, A., Eichberger, J. \& Grant, S. (2007), 'Choice under uncertainty with the best and worst in mind: Neo-additive capacities', Journal of Economic Theory $\mathbf{1 3 7}(1), 538-567$.

Chen, Y., Dimitrov, S., Sami, R., Reeves, D. M., Pennock, D. M., Hanson, R. D., Fortnow, L. \& Gonen, R. (2010), 'Gaming prediction markets: Equilibrium strategies with a market maker', Algorithmica 58(4), 930-969.

Chen, Y., Ruberry, M. \& Vaughan, J. W. (2012), 'Designing informative securities', arXiv preprint arXiv:1210.4837.

Condie, S. \& Ganguli, J. V. (2011), 'Ambiguity and rational expectations equilibria', The Review of Economic Studies 78(3), 821-845.

DeMarzo, P. \& Skiadas, C. (1998), 'Aggregation, determinacy, and informational efficiency for a class of economies with asymmetric information', journal of economic theory 80(1), 123-152.

DeMarzo, P. \& Skiadas, C. (1999), 'On the uniqueness of fully informative rational expectations equilibria', Economic Theory 13(1), 1-24.

Dimitrov, S. \& Sami, R. (2008), Non-myopic strategies in prediction markets, in 'Proceedings of the 9th ACM conference on Electronic commerce', ACM, pp. 200-209.

Dominiak, A. \& Lee, M. S. (n.d.), 'Dempster-shafer equilibrium and ambiguous signals'.

Dominiak, A. \& Lefort, J.-P. (2011), 'Unambiguous events and dynamic choquet preferences', Economic theory 46(3), 401-425.

Dominiak, A. \& Lefort, J.-P. (2013), 'Agreement theorem for neo-additive beliefs', Economic Theory $\mathbf{5 2}(1), 1-13$.

Dominiak, A. \& Lefort, J.-P. (2015), 'agreeing to disagree type results under ambiguity', Journal of Mathematical Economics 61, 119-129.

Dubey, P., Geanakoplos, J. \& Shubik, M. (1987), 'The revelation of information in strategic market games: A critique of rational expectations equilibrium', Journal of Mathematical Economics 16(2), 105-137.

Eichberger, J. \& Kelsey, D. (2004), 'Sequential two-player games with ambiguity', International Economic Review 45(4), 1229-1261.

Fischbacher, U. (2007), 'z-tree: Zurich toolbox for ready-made economic experiments', Experimental economics 10(2), 171-178.

Forsythe, R. \& Lundholm, R. (1990), 'Information aggregation in an experimental market', Econometrica: Journal of the Econometric Society pp. 309-347. 
Fréchette, G. R. \& Yuksel, S. (2013), 'Infinitely repeated games in the laboratory: Four perspectives on discounting and random termination', Available at SSRN 2225331.

Fudenberg, D. \& Tirole, J. (1991), 'Perfect bayesian equilibrium and sequential equilibrium', journal of Economic Theory 53(2), 236-260.

Geanakoplos, J. (1995), 'Common knowledge. handbook of game theory, vol. 2'.

Geanakoplos, J. D. \& Polemarchakis, H. M. (1982), 'We can’t disagree forever', Journal of Economic Theory 28(1), 192-200.

Gilboa, I. \& Schmeidler, D. (1989), 'Maxmin expected utility with non-unique prior', Journal of mathematical economics 18(2), 141-153.

Golosov, M., Lorenzoni, G. \& Tsyvinski, A. (2014), 'Decentralized trading with private information', Econometrica 82(3), 1055-1091.

Grossman, S. (1976), 'On the efficiency of competitive stock markets where trades have diverse information', The Journal of Finance 31(2), 573-585.

Hanany, E. \& Klibanoff, P. (2009), 'Updating ambiguity averse preferences', The BE Journal of Theoretical Economics $\mathbf{9}(1)$.

Hanany, E., Klibanoff, P. \& Mukerji, S. (n.d.), 'Incomplete information games with smooth ambiguity preferences'.

Hanson, R. (2003), 'Combinatorial information market design', Information Systems Frontiers 5(1), 107-119.

Hanson, R., Oprea, R. \& Porter, D. (2006), 'Information aggregation and manipulation in an experimental market', Journal of Economic Behavior $\&$ Organization 60(4), 449-459.

Hayek, F. A. (1945), 'The use of knowledge in society', The American economic review pp. 519-530.

Hellwig, M. F. (1982), 'Rational expectations equilibrium with conditioning on past prices: A mean-variance example', Journal of Economic Theory 26(2), 279-312.

Jian, L. \& Sami, R. (2012), 'Aggregation and manipulation in prediction markets: effects of trading mechanism and information distribution', Management Science 58(1), 123140 .

Kajii, A. \& Ui, T. (2005), 'Incomplete information games with multiple priors*', Japanese Economic Review 56(3), 332-351.

Kajii, A. \& Ui, T. (2009), 'Interim efficient allocations under uncertainty', Journal of Economic Theory 144(1), 337-353. 
Kellner, C. \& Thordal-Le Quement, M. (n.d.), 'Endogenous ambiguity in cheap talk'.

Kyle, A. S. (1985), 'Continuous auctions and insider trading', Econometrica: Journal of the Econometric Society pp. 1315-1335.

Lopomo, G., Rigotti, L. \& Shannon, C. (2009), 'Uncertainty in mechanism design', Revise and resubmit at Review of Economic Studies .

McKelvey, R. D. \& Page, T. (1986a), 'Common knowledge, consensus, and aggregate information', Econometrica: Journal of the Econometric Society pp. 109-127.

McKelvey, R. D. \& Page, T. (1986b), 'Common knowledge, consensus, and aggregate information', Econometrica: Journal of the Econometric Society pp. 109-127.

McKelvey, R. D. \& Page, T. (1990), 'Public and private information: An experimental study of information pooling', Econometrica: Journal of the Econometric Society pp. 1321-1339.

Mele, A. \& Sangiorgi, F. (2015), 'Uncertainty, information acquisition and price swings in asset markets', The Review of Economic Studies p. rdv017.

Milgrom, P. \& Stokey, N. (1982), 'Information, trade and common knowledge', Journal of Economic Theory 26(1), 17-27.

Nielsen, L. T. (1984), 'Common knowledge, communication, and convergence of beliefs', Mathematical Social Sciences 8(1), 1-14.

Nielsen, L. T., Brandenburger, A., Geanakoplos, J., McKelvey, R. \& Page, T. (1990), 'Common knowledge of an aggregate of expectations', Econometrica: Journal of the Econometric Society pp. 1235-1239.

Ostrovsky, M. (2012), 'Information aggregation in dynamic markets with strategic traders', Econometrica 80(6), 2595-2647.

Pires, C. P. (2002), 'A rule for updating ambiguous beliefs', Theory and Decision 53(2), 137-152.

Plott, C. R. \& Sunder, S. (1988), 'Rational expectations and the aggregation of diverse information in laboratory security markets', Econometrica: Journal of the Econometric Society pp. 1085-1118.

Plott, C. R., Wit, J. \& Yang, W. C. (2003), 'Parimutuel betting markets as information aggregation devices: experimental results', Economic Theory 22(2), 311-351.

Radner, R. (1979), 'Rational expectations equilibrium: Generic existence and the information revealed by prices', Econometrica: Journal of the Econometric Society pp. $655-678$. 
Sass, L. (2013), 'Kuhn's theorem for extensive form ellsberg games', Institute of Mathematical Economics Working Paper (478).

Schmeidler, D. (1989), 'Subjective probability and expected utility without additivity', Econometrica: Journal of the Econometric Society pp. 571-587.

Sebenius, J. K. \& Geanakoplos, J. (1983), 'Don't bet on it: Contingent agreements with asymmetric information', Journal of the American Statistical Association 78(382), 424-426.

Siniscalchi, M. (2006), 'Dynamic choice under ambiguity', Available at SSRN 948928.

Vespa, E. (2011), 'An experimental investigation of strategies in the dynamic common pool game', Available at SSRN 1961450 .

Wolinsky, A. (1990), 'Information revelation in a market with pairwise meetings', Econometrica: Journal of the Econometric Society pp. 1-23.

Wolitzky, A. (2014), 'Mechanism design with maxmin agents: Theory and an application to bilateral trade', manuscript, Stanford University . 